\title{
Antiplatelet and anticoagulant agents for secondary prevention of stroke and other thromboembolic events in people with antiphospholipid syndrome (Review)
}

Bala MM, Celinska-Lowenhoff M, Szot W, Padjas A, Kaczmarczyk M, Swierz MJ, Undas A

Bala MM, Celinska-Lowenhoff M, Szot W, Padjas A, Kaczmarczyk M, Swierz MJ, Undas A.

Antiplatelet and anticoagulant agents for secondary prevention of stroke and other thromboembolic events in people with antiphospholipid syndrome.

Cochrane Database of Systematic Reviews 2020, Issue 10. Art. No.: CD012169.

DOI: 10.1002/14651858.CD012169.pub3. 
TABLE OF CONTENTS

HEADER

ABSTRACT

PLAIN LANGUAGE SUMMARY

SUMMARY OF FINDINGS

BACKGROUND

OBJECTIVES

METHODS

RESULTS

Figure 1.

Figure 2.

Figure 3.

Figure 4.

DISCUSSION

AUTHORS' CONCLUSIONS

ACKNOWLEDGEMENTS

REFERENCES

CHARACTERISTICS OF STUDIES

DATA AND ANALYSES

Analysis 1.1. Comparison 1: NOAC (rivaroxaban) versus standard dose VKA, Outcome 1: Any thromboembolic event at the longest follow-up

Analysis 1.2. Comparison 1: NOAC (rivaroxaban) versus standard dose VKA, Outcome 2: Major bleeding at the longest followup

Analysis 1.3. Comparison 1: NOAC (rivaroxaban) versus standard dose VKA, Outcome 3: Major bleeding at the longest followup - calculated from log hazard ratio

Analysis 1.4. Comparison 1: NOAC (rivaroxaban) versus standard dose VKA, Outcome 4: All-cause mortality at the longest followup

Analysis 1.5. Comparison 1: NOAC (rivaroxaban) versus standard dose VKA, Outcome 5: Stroke at the longest follow-up .......... Analysis 1.6. Comparison 1: NOAC (rivaroxaban) versus standard dose VKA, Outcome 6: TIA at the longest follow-up .............. Analysis 1.7. Comparison 1: NOAC (rivaroxaban) versus standard dose VKA, Outcome 7: VTE at the longest follow-up .............. Analysis 1.8. Comparison 1: NOAC (rivaroxaban) versus standard dose VKA, Outcome 8: Myocardial infarction at the longest follow-up

Analysis 1.9. Comparison 1: NOAC (rivaroxaban) versus standard dose VKA, Outcome 9: Other thrombotic events at the longest follow-up

Analysis 1.10. Comparison 1: NOAC (rivaroxaban) versus standard dose VKA, Outcome 10: Mean Quality of life at day $180 \quad \ldots . . .$.

Analysis 1.11. Comparison 1: NOAC (rivaroxaban) versus standard dose VKA, Outcome 11: Clinically relevant non-major bleeding at the longest follow up

Analysis 1.12. Comparison 1: NOAC (rivaroxaban) versus standard dose VKA, Outcome 12: Minor bleeding at the longest followup

Analysis 2.1. Comparison 2: High dose VKA versus standard dose VKA, Outcome 1: Any thromboembolic event at the longest follow-up

Analysis 2.2. Comparison 2: High dose VKA versus standard dose VKA, Outcome 2: Any thromboembolic event at the longest follow-up - calculated from log hazard ratio

Analysis 2.3. Comparison 2: High dose VKA versus standard dose VKA, Outcome 3: Major bleeding at the longest follow-up .... Analysis 2.4. Comparison 2: High dose VKA versus standard dose VKA, Outcome 4: Major bleeding at the longest follow-up calculated from log hazard ratio

Analysis 2.5. Comparison 2: High dose VKA versus standard dose VKA, Outcome 5: All-cause mortality at the longest follow-up . Analysis 2.6. Comparison 2: High dose VKA versus standard dose VKA, Outcome 6: Stroke at the longest follow-up ................. Analysis 2.7. Comparison 2: High dose VKA versus standard dose VKA, Outcome 7: TIA at the longest follow-up ...................... Analysis 2.8. Comparison 2: High dose VKA versus standard dose VKA, Outcome 8: VTE at the longest follow-up ..................... Analysis 2.9. Comparison 2: High dose VKA versus standard dose VKA, Outcome 9: Myocardial infarction at the longest followup

Analysis 2.10. Comparison 2: High dose VKA versus standard dose VKA, Outcome 10: Other thrombotic events at the longest follow-up (superficial vein thrombosis) 
Analysis 2.11. Comparison 2: High dose VKA versus standard dose VKA, Outcome 11: Minor bleeding at the longest follow-up ..

Analysis 2.12. Comparison 2: High dose VKA versus standard dose VKA, Outcome 12: Any bleeding at the longest follow-up .... Analysis 2.13. Comparison 2: High dose VKA versus standard dose VKA, Outcome 13: Any bleeding at the longest follow-up calculated from log hazard ratio

Analysis 3.1. Comparison 3: Standard dose VKA plus single antiplatelet agent versus standard dose VKA, Outcome 1: Any thromboembolic event at the longest follow up

Analysis 3.2. Comparison 3: Standard dose VKA plus single antiplatelet agent versus standard dose VKA, Outcome 2: Major bleeding at the longest follow up

Analysis 3.3. Comparison 3: Standard dose VKA plus single antiplatelet agent versus standard dose VKA, Outcome 3: All-cause mortality at the longest follow up

Analysis 3.4. Comparison 3: Standard dose VKA plus single antiplatelet agent versus standard dose VKA, Outcome 4: Stroke at the longest follow-up

Analysis 3.5. Comparison 3: Standard dose VKA plus single antiplatelet agent versus standard dose VKA, Outcome 5: TIA at the longest follow-up

Analysis 3.6. Comparison 3: Standard dose VKA plus single antiplatelet agent versus standard dose VKA, Outcome 6: VTE at the longest follow-up

Analysis 3.7. Comparison 3: Standard dose VKA plus single antiplatelet agent versus standard dose VKA, Outcome 7: Myocardial infarction at the longest follow-up

Analysis 3.8. Comparison 3: Standard dose VKA plus single antiplatelet agent versus standard dose VKA, Outcome 8: Other thrombotic events at the longest follow-up (hearing loss, retinal artery thrombosis)

Analysis 3.9. Comparison 3: Standard dose VKA plus single antiplatelet agent versus standard dose VKA, Outcome 9: Minor bleeding at the longest follow-up

Analysis 4.1. Comparison 4: Standard dose VKA plus single antiplatelet agent versus single antiplatelet agent, Outcome 1: Major bleeding (minor cerebral haemorrhage) at a mean of 3.9 years

Analysis 4.2. Comparison 4: Standard dose VKA plus single antiplatelet agent versus single antiplatelet agent, Outcome 2: Stroke at 1-year follow-up

Analysis 4.3. Comparison 4: Standard dose VKA plus single antiplatelet agent versus single antiplatelet agent, Outcome 3: Minor bleeding (subcutaneous haemorrhage) at a mean of 3.9 years

Analysis 4.4. Comparison 4: Standard dose VKA plus single antiplatelet agent versus single antiplatelet agent, Outcome 4: GI bleeding (no definition) at a mean of 3.9 years

Analysis 5.1. Comparison 5: Standard dose VKA plus antiplatelet agent vs dual antiplatelet therapy, Outcome 1: Stroke at 3 years

Analysis 6.1. Comparison 6: Dual antiplatelet therapy vs single antiplatelet agent, Outcome 1: Stroke at 1 year ...................... Analysis 7.1. Comparison 7: Sensitivity analysis NOAC vs VKA, Outcome 1: Best case any thromboembolic event at the longest follow-up

Analysis 7.2. Comparison 7: Sensitivity analysis NOAC vs VKA, Outcome 2: Worst case any thromboembolic event at the longest follow-up

Analysis 7.3. Comparison 7: Sensitivity analysis NOAC vs VKA, Outcome 3: Best case major bleeding at the longest follow-up ... Analysis 7.4. Comparison 7: Sensitivity analysis NOAC vs VKA, Outcome 4: Worst case major bleeding at the longest follow-up . Analysis 7.5. Comparison 7: Sensitivity analysis NOAC vs VKA, Outcome 5: Best case all-cause death at the longest follow-up .. Analysis 7.6. Comparison 7: Sensitivity analysis NOAC vs VKA, Outcome 6: Worst case all-cause death at the longest follow-up . Analysis 7.7. Comparison 7: Sensitivity analysis NOAC vs VKA, Outcome 7: Best case clinically relevant non-major bleeding at the longest follow up

Analysis 7.8. Comparison 7: Sensitivity analysis NOAC vs VKA, Outcome 8: Worst case clinically relevant non-major bleeding at the longest follow up

Analysis 7.9. Comparison 7: Sensitivity analysis NOAC vs VKA, Outcome 9: Best case minor bleeding at the longest follow-up .. Analysis 7.10. Comparison 7: Sensitivity analysis NOAC vs VKA, Outcome 10: Worst case minor bleeding at the longest followup

APPENDICES

WHAT'S NEW

HISTORY 
Copyright $\odot 2020$ The Cochrane Collaboration. Published by John Wiley \& Sons, Ltd. 
[Intervention Review]

\section{Antiplatelet and anticoagulant agents for secondary prevention of stroke and other thromboembolic events in people with antiphospholipid syndrome}

Malgorzata M Bala1,2, Magdalena Celinska-Lowenhoff ${ }^{3}$, Wojciech Szot ${ }^{1}$, Agnieszka Padjas ${ }^{3}$, Mateusz Kaczmarczyk ${ }^{4}$, Mateusz J Swierz ${ }^{1,2}$, Anetta Undas 5

${ }^{1}$ Chair of Epidemiology and Preventive Medicine, Department of Hygiene and Dietetics, Jagiellonian University Medical College, Krakow, Poland. 2Systematic Reviews Unit, Jagiellonian University Medical College, Krakow, Poland. 32nd Department of Internal Medicine, Department of Allergy and Immunology, Jagiellonian University Medical College, Krakow, Poland. ${ }^{4}$ Systematic Reviews Unit - Polish Cochrane Branch, Jagiellonian University Medical College, Krakow, Poland. 5Institute of Cardiology, Jagiellonian University Medical College, Krakow, Poland

Contact address: Malgorzata M Bala,gosiabala@mp.pl, malgorzata.1.bala@uj.edu.pl.

Editorial group: Cochrane Stroke Group.

Publication status and date: New search for studies and content updated (conclusions changed), published in Issue 10, 2020.

Citation: Bala MM, Celinska-Lowenhoff M, Szot W, Padjas A, Kaczmarczyk M, Swierz MJ, Undas A. Antiplatelet and anticoagulant agents for secondary prevention of stroke and other thromboembolic events in people with antiphospholipid syndrome. Cochrane Database of Systematic Reviews 2020, Issue 10. Art. No.: CD012169. DOI: 10.1002/14651858.CD012169.pub3.

Copyright ( 2020 The Cochrane Collaboration. Published by John Wiley \& Sons, Ltd.

\section{A B S T R A C T}

\section{Background}

Antiphospholipid syndrome (APS) is a systemic autoimmune disease characterized by arterial or venous thrombosis (or both), and/or pregnancy morbidity in association with the presence of antiphospholipid antibodies. The prevalence of APS is estimated at 40 to 50 cases per 100,000 people. The most common sites of thrombosis are cerebral arteries and deep veins of the lower limbs. People with a definite APS diagnosis have an increased lifetime risk of recurrent thrombotic events.

\section{Objectives}

To assess the effects of antiplatelet (AP) or anticoagulant agents, or both, for the secondary prevention of recurrent thrombosis, particularly ischemic stroke, in people with APS.

\section{Search methods}

We last searched the MEDLINE, Embase, CENTRAL, Cochrane Stroke Group Trials Register, and ongoing trials registers on 22 November 2019. We checked reference lists of included studies, systematic reviews, and practice guidelines. We also contacted experts in the field.

\section{Selection criteria}

We included randomized controlled trials (RCTs) that evaluated any anticoagulant or AP agent, or both, in the secondary prevention of thrombosis in people with APS, according to the criteria valid when the study took place. We did not include studies specifically addressing women with obstetrical APS.

\section{Data collection and analysis}

Pairs of review authors independently worked on each step of the review, following Cochrane methods. We summarized the evidence using the GRADE approach. 


\section{Main results}

We identified eight studies including 811 participants that compared different AP or anticoagulant agents.

NOAC (non-VKA oral anticoagulant: rivaroxaban 15 or $20 \mathrm{mg} / \mathrm{d}$ ) versus standard-dose VKA (vitamin $\mathrm{K}$ antagonist: warfarin at moderate International Normalized Ratio [INR] - 2.5) or adjusted [INR 2.0-3.0] dose):

In three studies there were no differences in any thromboembolic event (including death) and major bleeding (moderate-certainty evidence), but an increased risk of stroke (risk ratio [RR] 14.13, 95\% confidence interval [CI] 1.87 to 106.8; moderate-certainty evidence). One of the studies reported a small benefit of rivaroxaban in terms of quality of life at 180 days measured as health state on Visual Analogue Scale (mean difference [MD] $7 \mathrm{~mm}, 95 \% \mathrm{Cl} 2.01$ to 11.99 ; low-certainty evidence), but not measured as health utility on a scale from 0 to 1 (MD $0.04,95 \% \mathrm{Cl}-0.02$ to 0.10 ; low-certainty evidence).

High-dose VKA (warfarin with a target INR of 3.1 to 4.0 [mean 3.3] or 3.5 [mean 3.2]) versus standard-dose VKA (warfarin with a target INR of 2.0 to 3.0 [mean 2.3] or 2.5 [mean 2.5]):

In two studies there were no differences in the rates of thrombotic events and major bleeding (RR 2.22, $95 \% \mathrm{Cl} 0.79$ to 6.23 , low-certainty evidence), but an increased risk of minor bleeding in one study during a mean of 3.4 years (standard deviation [SD] 1.2) of follow-up (RR $2.55,95 \% \mathrm{Cl} 1.07$ to 6.07 ). In both trials there was evidence of a higher risk of any bleeding (hazard ratio [HR] $2.0395 \% \mathrm{Cl} 1.12$ to 3.68 ; lowcertainty evidence) in the high-dose VKA group, and for this outcome (any bleeding) the incidence is not different, only the time to event is showing an effect.

Standard-dose VKA plus a single AP agent (warfarin at a target INR of 2.0 to 3.0 plus aspirin $100 \mathrm{mg} / \mathrm{d}$ ) versus standard-dose VKA (warfarin at a target INR of 2.0 to 3.0 ):

One high-risk-of-bias study showed an increased risk of any thromboembolic event with combined treatment (RR 2.14, 95\% Cl 1.04 to 4.43; low-certainty evidence) and reported on major bleeding with five cases in the combined treatment group and one case in the standarddose VKA treatment group, resulting in RR 7.42 (95\% Cl 0.91 to 60.7 ; low-certainty evidence) and no differences for secondary outcomes (very low- to low-certainty evidence).

Single/dual AP agent and standard-dose VKA (pooled results):

Two high-risk-of-bias studies compared a combination of AP and VKA (aspirin $100 \mathrm{mg} / \mathrm{d}$ plus warfarin or unspecified VKA at a target INR of 2.0 to 3.0 or 2.0 to 2.5) with a single AP agent (aspirin $100 \mathrm{mg} / \mathrm{d}$ ), but did not provide any conclusive evidence regarding the effects of those drugs in people with APS (very low-certainty evidence).

One of the above-mentioned studies was a three-armed study that compared a combination of AP and VKA (aspirin $100 \mathrm{mg} / \mathrm{d}$ plus warfarin at a target INR of 2.0 to 2.5) with dual AP therapy (aspirin $100 \mathrm{mg} / \mathrm{d}$ plus cilostazol $200 \mathrm{mg} / \mathrm{d}$ ) and dual AP therapy (aspirin $100 \mathrm{mg} / \mathrm{d}$ plus cilostazol $200 \mathrm{mg} / \mathrm{d}$ ) versus a single AP treatment (aspirin $100 \mathrm{mg} / \mathrm{d}$ ). This study reported on stroke (very low-certainty evidence) but did not report on any thromboembolic events, major bleeding, or any secondary outcomes.

We identified two ongoing studies and three studies are awaiting classification.

\section{Authors' conclusions}

The evidence identified indicates that NOACs compared with standard-dose VKAs may increase the risk of stroke and do not appear to alter the risk of other outcomes (moderate-certainty evidence). Using high-dose VKA versus standard-dose VKA did not alter the risk of any thromboembolic event or major bleeding but may increase the risk of any form of bleeding (low-certainty evidence). Standard-dose VKA combined with an AP agent compared with standard-dose VKA alone may increase the risk of any thromboembolic event and does not appear to alter the risk of major bleeding or other outcomes (low-certainty evidence).

The evidence is very uncertain about the benefit or harm of using standard-dose VKA plus AP agents versus single or dual AP therapy, or dual versus single AP therapy, for the secondary prevention of recurrent thrombosis in people with APS (very low-certainty evidence).

\section{PLAIN LANGUAGE SUMMARY}

\section{Anticoagulant drugs or antiplatelet drugs, or both, for reducing the risk of blood clots and stroke in people with antiphospholipid syndrome}

\section{Review question}

This review aimed to find out which type of treatment works best for preventing stroke and other blood clotting (thrombotic) events in people with antiphospholipid syndrome (APS).

\section{Background}


APS is a disease where the immune system produces antibodies against the person's own cells. The presence of such antibodies increases the risk of developing blood clots (thrombosis) in blood vessels (veins or arteries, or both) and can cause pregnancy-related complications such as miscarriage. Blood clots in arteries can cause strokes. Two types of drugs are commonly used to prevent blood clots in people with APS: anticoagulants such as warfarin (which interfere with proteins that form blood clots) and antiplatelet drugs such as aspirin (which prevent cells called platelets from sticking together).

\section{Study characteristics}

The evidence is current to 22 November 2019. We looked for studies that randomly allocated people with APS to different treatments, including anticoagulants, antiplatelet drugs, or both. We identified eight studies involving 811 participants, with the average age between 36 and 50 years. Three studies compared rivaroxaban (a new oral anticoagulant) with standard-dose warfarin (a vitamin $\mathrm{K}$ antagonist VKA). Two studies compared two intensities of VKA anticoagulation: high versus standard. One study compared standard-dose VKA plus aspirin versus standard-dose VKA alone. The remaining studies compared different combinations of anticoagulants or antiplatelet drugs (e.g. aspirin). One study compared dual antiplatelet drugs versus single antiplatelet drugs. Interventions lasted from 180 days to a median of 58.4 months.

\section{Key results}

In three studies comparing rivaroxaban (NOAC) with standard-dose VKA there were similar proportions of participants with blood clots, bleeding, or death (moderate-certainty evidence), but there were more participants with stroke in the NOAC group than in the VKA group (moderate-certainty evidence). In the two studies comparing high-dose versus a standard-dose VKA, similar proportions of participants had blood clotting and major bleeding problems (low-certainty evidence), but participants in the high-dose group had a greater risk of minor and any bleeding problems (low-certainty evidence). A study comparing standard VKA plus an antiplatelet drug with standard-dose VKA observed more participants with any thromboembolic event with combined treatment (low-certainty evidence) and similar proportions of participants for other outcomes (very low- to low-certainty evidence).

The remaining studies comparing different combinations of antiplatelet drug and/or VKA were inconclusive regarding benefits or harms (low- or very low-certainty evidence).

We identified two ongoing studies and three studies are awaiting classification.

\section{Certainty of the evidence}

Most of the results were imprecise and did not clearly indicate benefit or harm. 


\section{S U M MARY OF F I N D I G S}

Summary of findings 1. NOAC (rivaroxaban) versus standard-dose VKA

NOAC (rivaroxaban) versus standard-dose VKA

Patient or population: people with antiphospholipid syndrome and a history of stroke and or thromboembolic events Setting: specialists centres

Intervention: NOAC (rivaroxaban)

Comparison: standard-dose VKA

\begin{tabular}{|c|c|c|c|c|c|c|}
\hline \multirow[t]{2}{*}{ Outcomes } & \multicolumn{2}{|c|}{ Anticipated absolute effects* $(95 \% \mathrm{Cl})$} & \multirow{2}{*}{$\begin{array}{l}\text { Relative effect } \\
(95 \% \mathrm{CI})\end{array}$} & \multirow{2}{*}{$\begin{array}{l}\text { № of partici- } \\
\text { pants } \\
\text { (studies) }\end{array}$} & \multirow{2}{*}{$\begin{array}{l}\text { Certainty of } \\
\text { the evidence } \\
\text { (GRADE) }\end{array}$} & \multirow[t]{2}{*}{ Comments } \\
\hline & $\begin{array}{l}\text { Risk with stan- } \\
\text { dard-dose VKA }\end{array}$ & Risk with NOAC & & & & \\
\hline \multirow{2}{*}{$\begin{array}{l}\text { Any thromboembolic event } \\
\text { Follow-up: range } 7 \text { months to } 35.4 \text { months }\end{array}$} & \multicolumn{2}{|l|}{ Study population } & \multirow{2}{*}{$\begin{array}{l}\text { RR } 4.08 \\
\text { (0.48 to } 34.79)\end{array}$} & \multirow{2}{*}{$\begin{array}{l}425 \\
\text { (3 RCTs) }\end{array}$} & \multirow{2}{*}{$\begin{array}{l}\oplus \oplus \oplus \ominus \\
\text { Moderateb }\end{array}$} & \\
\hline & 28 per 1000 & $\begin{array}{l}115 \text { per } 1000 \\
(14 \text { to } 975)\end{array}$ & & & & \\
\hline \multirow{2}{*}{$\begin{array}{l}\text { Major bleeding } \\
\text { Follow-up: range } 7 \text { months to } 35.4 \text { months }\end{array}$} & \multicolumn{2}{|l|}{ Study population } & \multirow{2}{*}{$\begin{array}{l}\text { RR } 1.10 \\
\text { (0.45 to } 2.68)\end{array}$} & \multirow{2}{*}{$\begin{array}{l}425 \\
\text { (3 RCTs) }\end{array}$} & \multirow{2}{*}{$\begin{array}{l}\oplus \oplus \oplus \ominus \\
\text { Moderate }\end{array}$} & \\
\hline & 42 per 1000 & $\begin{array}{l}47 \text { per } 1000 \\
(19 \text { to } 113)\end{array}$ & & & & \\
\hline \multirow{2}{*}{$\begin{array}{l}\text { All-cause mortality }{ }^{a} \\
\text { Follow-up: range } 7 \text { months to } 35.4 \text { months }\end{array}$} & \multicolumn{2}{|l|}{ Study population } & \multirow{2}{*}{$\begin{array}{l}\text { RR } 1.45 \\
\text { (0.44 to } 4.78)\end{array}$} & \multirow{2}{*}{$\begin{array}{l}425 \\
\text { (3 RCTs) }\end{array}$} & \multirow{2}{*}{$\begin{array}{l}\oplus \oplus \oplus \ominus \\
\text { Moderate } b\end{array}$} & \\
\hline & 19 per 1000 & $\begin{array}{l}28 \text { per } 1000 \\
\text { (9 to } 91)\end{array}$ & & & & \\
\hline \multirow{2}{*}{$\begin{array}{l}\text { Stroke }{ }^{a} \\
\text { Follow-up: range } 7 \text { months to } 35.4 \text { months }\end{array}$} & \multicolumn{2}{|l|}{ Study population } & \multirow{2}{*}{$\begin{array}{l}\text { RR } 14.13 \\
\text { (1.87 to } 106.81)\end{array}$} & \multirow{2}{*}{$\begin{array}{l}425 \\
\text { (3 RCTs) }\end{array}$} & \multirow{2}{*}{$\begin{array}{l}\oplus \oplus \oplus \ominus \\
\text { Moderatec }\end{array}$} & \\
\hline & 0 per 1000 & 0 per 1000 & & & & \\
\hline $\begin{array}{l}\text { Mean quality of life - health utility } \\
\text { Follow-up: } 180 \text { days }\end{array}$ & & $\begin{array}{l}\text { MD } 0.04 \text { higher } \\
\text { ( } 0.02 \text { lower to } 0.1 \text { higher) }\end{array}$ & - & $\begin{array}{l}111 \\
(1 \mathrm{RCT})\end{array}$ & $\begin{array}{l}\oplus \oplus \ominus \ominus \\
\text { Lowd,e }\end{array}$ & \\
\hline $\begin{array}{l}\text { Mean quality of life - health state } \\
\text { Follow-up: } 180 \text { days }\end{array}$ & & $\begin{array}{l}\text { MD } 7 \text { higher } \\
\text { ( } 2.01 \text { higher to } 11.99 \text { high- } \\
\text { er) }\end{array}$ & - & $\begin{array}{l}112 \\
(1 \mathrm{RCT})\end{array}$ & $\begin{array}{l}\oplus \oplus \ominus \ominus \\
\text { Lowd,e }\end{array}$ & \\
\hline
\end{tabular}




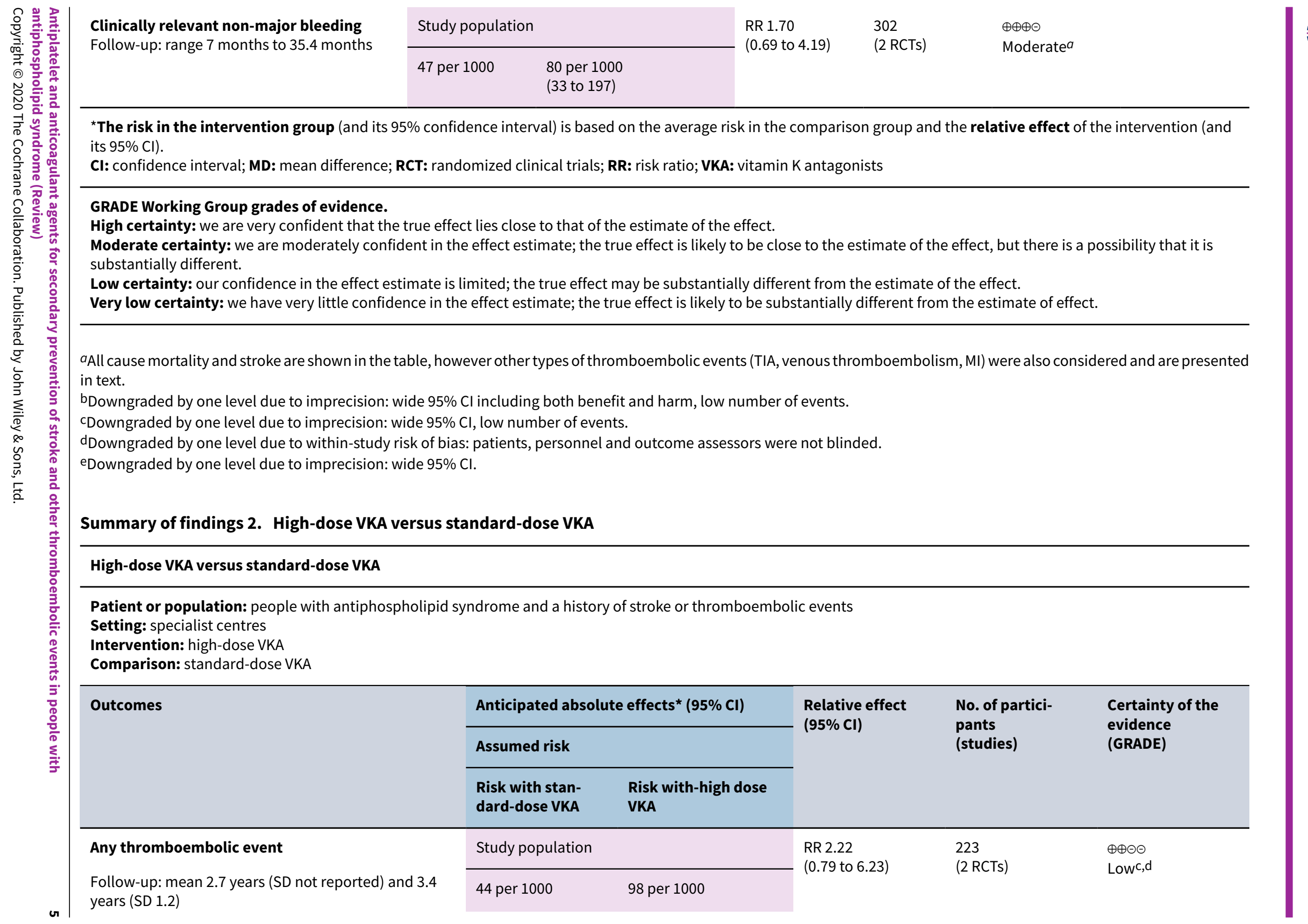




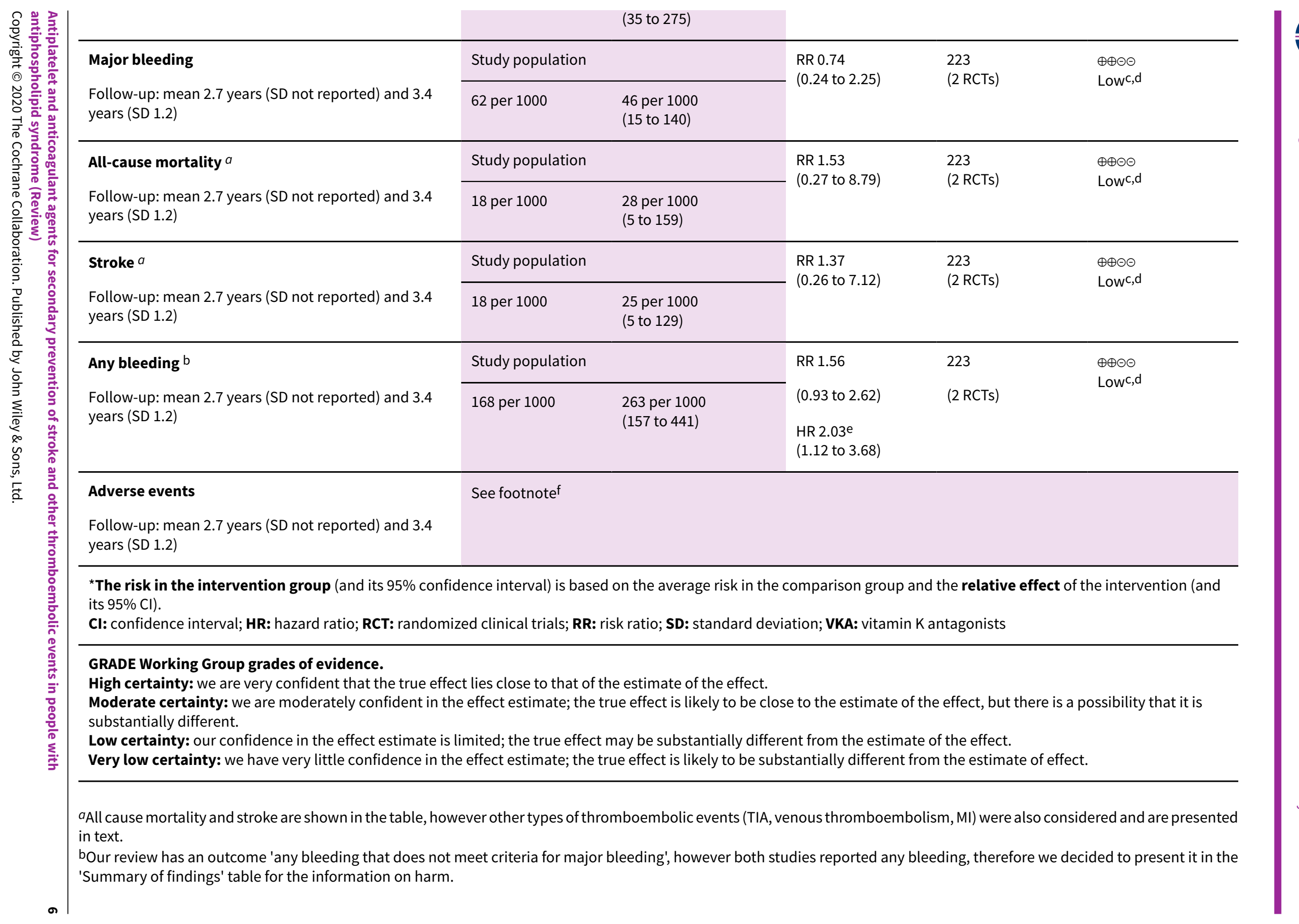


CDowngraded by one level due to within-study risk of bias: some issues concerning incomplete outcome reporting and selective outcome reporting; WAPS 2005 was seriously underpowered as was terminated early due to poor recruitment.

dDowngraded by one level due to imprecision: wide $95 \% \mathrm{Cl}$ including both benefit and harm, low number of events.

eThe results were not statistically significant when analyzed by RR; however when the time to event (HR) was taken into account there was a statistically significant difference between treatment groups.

fOnly one of the two included studies reported adverse events other than bleeding as outcomes and these adverse events lead to withdrawal from the study (WAPS 2005); these were essential thrombocythemia in one participant and headache in one participant, but the study did not indicate the group in which those participants were included.

\section{Summary of findings 3. Standard-dose VKA plus single antiplatelet agent versus standard-dose VKA}

\section{Standard-dose VKA plus single antiplatelet agent versus standard-dose VKA}

Patient or population: people with antiphospholipid syndrome and a history of stroke or thromboembolic events

Setting: specialist centres

Intervention: standard-dose VKA plus single antiplatelet agent

Comparison: standard-dose VKA

\begin{tabular}{|c|c|c|c|c|c|}
\hline \multirow[t]{3}{*}{ Outcomes } & \multicolumn{2}{|c|}{ Anticipated absolute effects ${ }^{\star}(95 \% \mathrm{Cl})$} & \multirow{3}{*}{$\begin{array}{l}\text { Relative effect } \\
(95 \% \mathrm{CI})\end{array}$} & \multirow{3}{*}{$\begin{array}{l}\text { No. of partici- } \\
\text { pants } \\
\text { (studies) }\end{array}$} & \multirow{3}{*}{$\begin{array}{l}\text { Certainty of the } \\
\text { evidence } \\
\text { (GRADE) }\end{array}$} \\
\hline & \multicolumn{2}{|l|}{ Assumed risk } & & & \\
\hline & $\begin{array}{l}\text { Risk with stan- } \\
\text { dard-dose VKA }\end{array}$ & $\begin{array}{l}\text { Risk with stan- } \\
\text { dard-dose VKA plus } \\
\text { single antiplatelet } \\
\text { agent }\end{array}$ & & & \\
\hline $\begin{array}{l}\text { Follow-up: median } 58.4 \text { months (IQR 0.9; 38.9), in VKA } \\
\text { +AP group follow-up and } 51.6 \text { (IQR 0.9; 48.8) in VKA } \\
\text { group }\end{array}$ & 184 per 1000 & $\begin{array}{l}394 \text { per } 1000 \\
\text { (192 to } 816)\end{array}$ & $\begin{array}{l}\text { RR } 2.14 \\
\text { (1.04 to } 4.43 \text { ) }\end{array}$ & $\begin{array}{l}82 \\
(1 \mathrm{RCTs})\end{array}$ & $\begin{array}{l}\oplus \oplus \ominus \ominus \\
\text { Lowc,d }\end{array}$ \\
\hline Major bleeding & \multicolumn{2}{|l|}{ Study population } & \multirow{2}{*}{$\begin{array}{l}\text { RR } 7.42 \\
\text { (0.91 to } 60.70)\end{array}$} & \multirow{2}{*}{$\begin{array}{l}82 \\
(1 \mathrm{RCTs})\end{array}$} & \multirow{2}{*}{$\begin{array}{l}\oplus \oplus \ominus \ominus \\
\text { Lowc,e }\end{array}$} \\
\hline $\begin{array}{l}\text { Follow-up: median } 58.4 \text { months (IQR 0.9; } 38.9 \text { ), in VKA } \\
\text { +AP group follow-up and } 51.6 \text { (IQR 0.9; 48.8) in VKA } \\
\text { group }\end{array}$ & 20 per 1000 & $\begin{array}{l}149 \text { per } 1000 \\
\text { (19 to } 1214 \text { ) }\end{array}$ & & & \\
\hline All-cause mortality ${ }^{a}$ & \multicolumn{2}{|l|}{ Study population } & \multirow{2}{*}{$\begin{array}{l}\text { RR } 0.49 \\
\text { (0.02 to } 11.68)\end{array}$} & \multirow{2}{*}{$\begin{array}{l}82 \\
(1 \mathrm{RCTS})\end{array}$} & \multirow{2}{*}{$\begin{array}{l}\oplus \odot \Theta \ominus \\
\text { Very lowc,f }\end{array}$} \\
\hline $\begin{array}{l}\text { Follow-up: median } 58.4 \text { months (IQR } 0.9 ; 38.9) \text {, in VKA } \\
\text { +AP group follow-up and } 51.6 \text { (IQR } 0.9 ; 48.8) \text { in VKA } \\
\text { group }\end{array}$ & 20 per 1000 & $\begin{array}{l}10 \text { per } 1000 \\
(1 \text { to } 234)\end{array}$ & & & \\
\hline
\end{tabular}




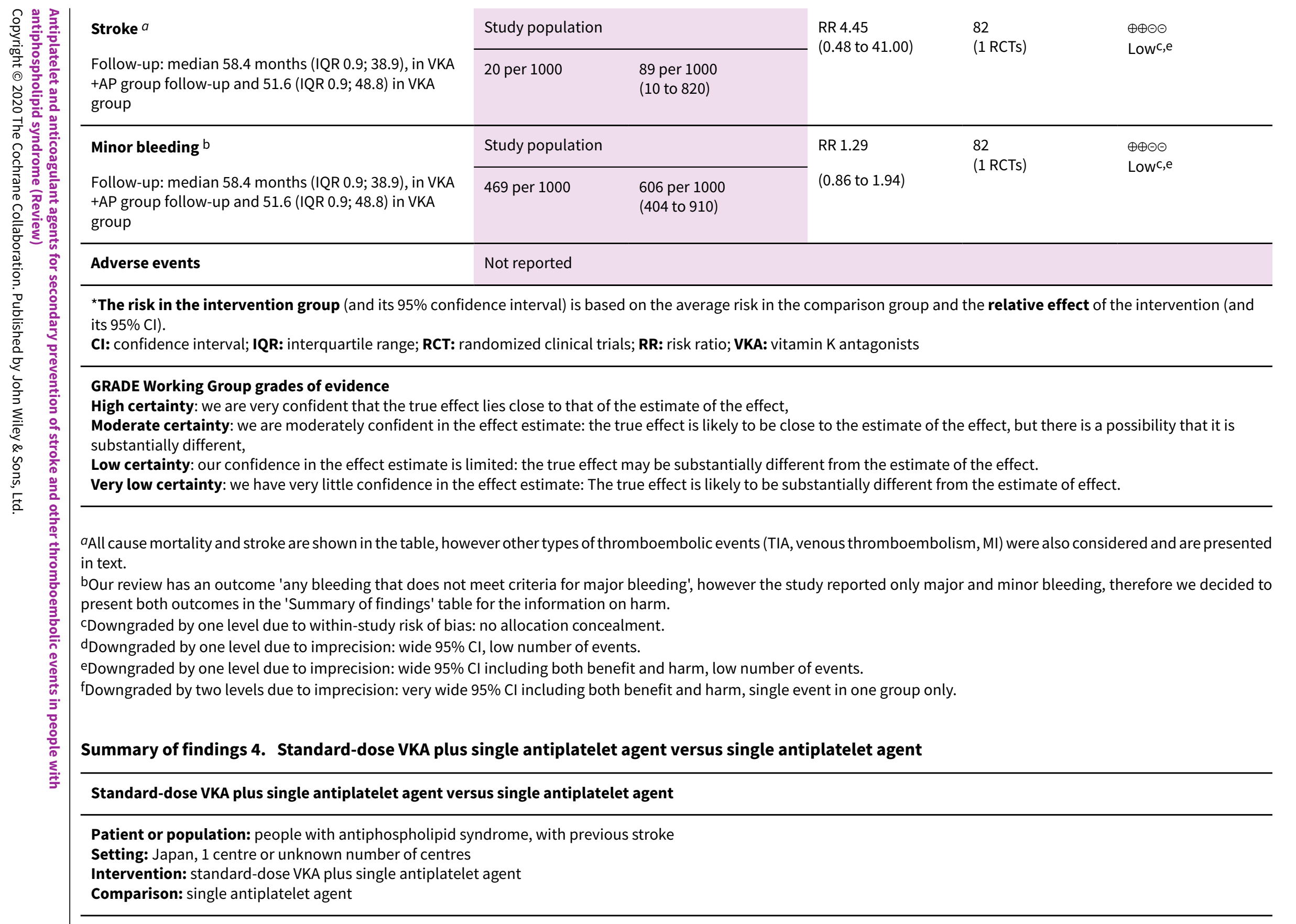




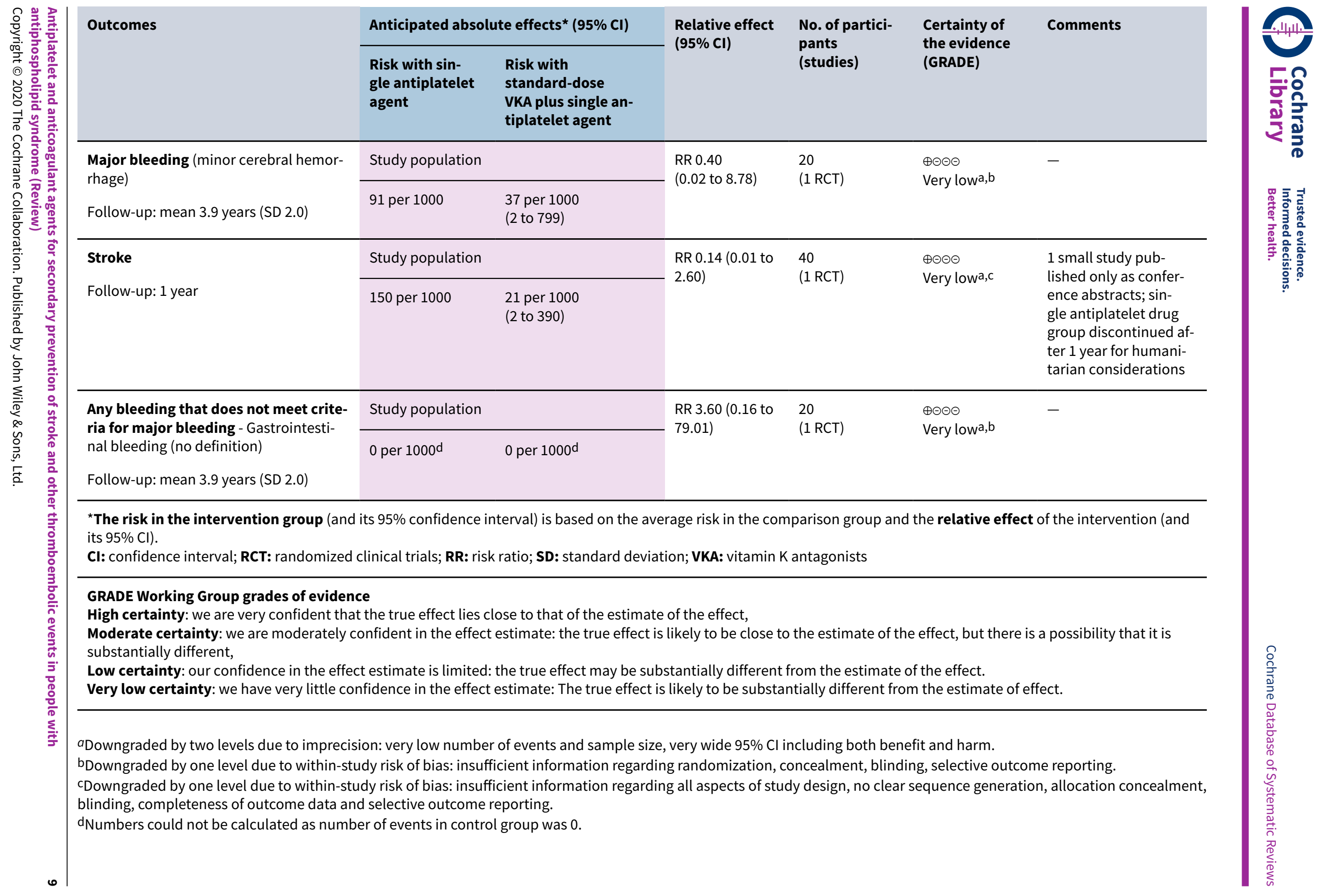




\section{Summary of findings 5. Standard-dose VKA plus single antiplatelet agent versus dual antiplatelet agent}

Standard-dose VKA plus single antiplatelet agent versus dual antiplatelet agent

Patient or population: people with antiphospholipid syndrome, with previous stroke

Setting: Japan, 1 centre

Intervention: standard-dose VKA plus single antiplatelet agent

Comparison: dual antiplatelet agent

\begin{tabular}{|c|c|c|c|c|c|c|}
\hline \multirow[t]{2}{*}{ Outcomes } & \multicolumn{2}{|c|}{ Anticipated absolute effects* $(95 \% \mathrm{Cl})$} & \multirow{2}{*}{$\begin{array}{l}\text { Relative effect } \\
(95 \% \mathrm{Cl})\end{array}$} & \multirow{2}{*}{$\begin{array}{l}\text { No. of partici- } \\
\text { pants } \\
\text { (studies) }\end{array}$} & \multirow{2}{*}{$\begin{array}{l}\text { Certainty of } \\
\text { the evidence } \\
\text { (GRADE) }\end{array}$} & \multirow[t]{2}{*}{ Comments } \\
\hline & $\begin{array}{l}\text { Risk with dual an- } \\
\text { tiplatelet agent }\end{array}$ & $\begin{array}{l}\text { Risk with } \\
\text { standard-dose VKA plus } \\
\text { single antiplatelet agent }\end{array}$ & & & & \\
\hline Stroke & \multicolumn{2}{|l|}{ Study population } & \multirow{2}{*}{$\begin{array}{l}\text { RR } 5.00 \text { (0.26 to } \\
98.00)\end{array}$} & \multirow{2}{*}{$\begin{array}{l}40 \\
(1 \mathrm{RCT})\end{array}$} & \multirow{2}{*}{$\begin{array}{l}\oplus \ominus \ominus \ominus \\
\text { Very lowa,b }\end{array}$} & \multirow{2}{*}{$\begin{array}{l}1 \text { small study published only as confer- } \\
\text { ence abstracts; single antiplatelet drug } \\
\text { group discontinued after } 1 \text { year for hu- } \\
\text { manitarian considerations }\end{array}$} \\
\hline $\begin{array}{l}\text { Follow-up: } 3 \\
\text { years }\end{array}$ & 0 per $1000^{c}$ & 0 per $1000 c$ & & & & \\
\hline
\end{tabular}

${ }^{\star}$ The risk in the intervention group (and its $95 \%$ confidence interval) is based on the average risk in the comparison group and the relative effect of the intervention (and its $95 \% \mathrm{Cl}$ ).

Cl: confidence interval; RCT: randomized clinical trials; RR: risk ratio; VKA: vitamin K antagonists

\section{GRADE Working Group grades of evidence}

High certainty: we are very confident that the true effect lies close to that of the estimate of the effect,

Moderate certainty: we are moderately confident in the effect estimate: the true effect is likely to be close to the estimate of the effect, but there is a possibility that it is substantially different,

Low certainty: our confidence in the effect estimate is limited: the true effect may be substantially different from the estimate of the effect.

Very low certainty: we have very little confidence in the effect estimate: The true effect is likely to be substantially different from the estimate of effect.

aDowngraded by two levels due to imprecision: very low number of events and sample size, very wide $95 \% \mathrm{Cl}$ including both benefit and harm.

bDowngraded by one level due to within-study risk of bias: insufficient information regarding all aspects of study design, no clear sequence generation, allocation concealment,

blinding, completeness of outcome data and selective outcome reporting.

cNumbers could not be calculated as number of events in control group was 0 .

\section{Summary of findings 6. Dual antiplatelet agent versus single antiplatelet agent}

\section{Dual antiplatelet agent versus single antiplatelet agent}

Patient or population: people with antiphospholipid syndrome, with previous stroke

Setting: Japan, 1 centre

เ

Intervention: dual antiplatelet agent 
Comparison: single antiplatelet agent

\begin{tabular}{|c|c|c|c|c|c|c|}
\hline \multirow[t]{2}{*}{ Outcomes } & \multicolumn{2}{|c|}{ Anticipated absolute effects ${ }^{\star}(95 \% \mathrm{Cl})$} & \multirow{2}{*}{$\begin{array}{l}\text { Relative effect } \\
(95 \% \mathrm{CI})\end{array}$} & \multirow{2}{*}{$\begin{array}{l}\text { No. of partici- } \\
\text { pants } \\
\text { (studies) }\end{array}$} & \multirow{2}{*}{$\begin{array}{l}\text { Certainty of } \\
\text { the evidence } \\
\text { (GRADE) }\end{array}$} & \multirow[t]{2}{*}{ Comments } \\
\hline & $\begin{array}{l}\text { Risk with dual an- } \\
\text { tiplatelet agent }\end{array}$ & $\begin{array}{l}\text { Risk with } \\
\text { single antiplatelet } \\
\text { agent }\end{array}$ & & & & \\
\hline Stroke & \multicolumn{2}{|l|}{ Study population } & \multirow{2}{*}{$\begin{array}{l}\text { RR } 0.14 \text { ( } 0.01 \text { to } \\
2.6 \text { ) }\end{array}$} & \multirow{2}{*}{$\begin{array}{l}40 \\
(1 \mathrm{RCT})\end{array}$} & \multirow{2}{*}{$\begin{array}{l}\oplus \ominus \odot \odot \\
\text { Very lowa,b }\end{array}$} & \multirow{2}{*}{$\begin{array}{l}1 \text { small study published only as conference } \\
\text { abstracts; single antiplatelet drug group } \\
\text { discontinued after } 1 \text { year for humanitarian } \\
\text { considerations }\end{array}$} \\
\hline $\begin{array}{l}\text { Follow-up: } 1 \\
\text { year }\end{array}$ & 150 per 1000 & $\begin{array}{l}21 \text { per } 1000 \\
(2 \text { to } 390)\end{array}$ & & & & \\
\hline
\end{tabular}

${ }^{*}$ The risk in the intervention group (and its 95\% confidence interval) is based on the average risk in the comparison group and the relative effect of the intervention (and its $95 \% \mathrm{Cl}$ ).

Cl: confidence interval; $\mathbf{R C T}$ : randomized clinical trials; $\mathbf{R R}$ : risk ratio

\section{GRADE Working Group grades of evidence}

High certainty: we are very confident that the true effect lies close to that of the estimate of the effect,

Moderate certainty: we are moderately confident in the effect estimate: the true effect is likely to be close to the estimate of the effect, but there is a possibility that it is

substantially different,

Low certainty: our confidence in the effect estimate is limited: the true effect may be substantially different from the estimate of the effect.

Very low certainty: we have very little confidence in the effect estimate: The true effect is likely to be substantially different from the estimate of effect.

aDowngraded by two levels due to imprecision: very low number of events and sample size, very wide $95 \% \mathrm{Cl}$ including both benefit and harm.

bDowngraded by one level due to within-study risk of bias: insufficient information regarding all aspects of study design, no clear sequence generation, allocation concealment,

blinding, completeness of outcome data and selective outcome reporting. 


\section{B A C K G R O U N D}

\section{Description of the condition}

Antiphospholipid syndrome (APS) is an autoimmune condition where the presence of antiphospholipid (aPL) antibodies is associated with recurrent thrombosis (both arterial and venous), pregnancy morbidity, or both. The pathogenesis of APS involves the activation of monocytes, platelets, endothelial cells and complements, which induce thrombosis (Chighizola 2015; Giannakopoulos 2007; Giannakopoulos 2013). Primary APS is diagnosed in $53.1 \%$ of cases, while $36.2 \%$ of cases are secondary APS (associated with other autoimmune diseases, especially with systemic lupus erythematosus, or SLE) (Cervera 2002). In the general population, estimates of the prevalence of aPL antibodies range from $1 \%$ to $5 \%$ of otherwise healthy people (Petri 2000), up to $10 \%$ (George 2009). The prevalence is higher in people with rheumatoid arthritis (16\%) and SLE (30\% to 40\%) (George 2009). According to the AntiPhospholipid Syndrome Alliance for Clinical Trials and InternatiOnal Networking (APS ACTION) Group data, aPL prevalence in people with thrombotic events was $6 \%$ in women with pregnancy morbidity and $13 \%$ in people with stroke (Andreoli 2013).

The prevalence of APS is estimated at 40 to 50 cases per 100,000 people, and the incidence is about five new cases per 100,000 people per year (Gomez-Puerta 2014). The estimated association between aPL positivity and annual risk of thrombosis in people with no previous thrombosis is $0 \%$ to $4 \%$ (Erkan 2007), while in people with SLE the annual risk for thrombotic events is $2.5 \%$ to $3.8 \%$. However, $4 \%$ to $21 \%$ of people with thrombosis are positive for aPL antibodies (Lim 2006).

The diagnosis of APS is based on the 2006 modified classification criteria, which include relatively specific and the most common clinical and laboratory findings: that is, vascular thrombosis, pregnancy morbidity, or both, with the presence of lupus anticoagulant (LA) and/or anticardiolipin antibodies ( $\mathrm{aCL}$ ) and/ or anti-beta 2 glycoprotein-I antibodies (anti- $\beta_{2} \mathrm{GPI}$ ) in plasma in medium to high titers. Antibodies must be detected at least twice in a 12-week period. To confirm the diagnosis of APS, one clinical and one laboratory criterion must be fulfilled (Miyakis 2006). The previous classification criteria for APS, established in Sapporo in 1999 , did not include anti- $\beta_{2}$ GPI antibodies and set the minimum time between two measurements at six weeks (Wilson 1999).

Associated with thrombosis, aPL antibodies are a heterogeneous group of antibodies found in people with APS. The presence of LA in plasma is the strongest risk factor for both venous and arterial thrombosis (Galli 2003).

Thrombosis in APS may affect both venous and arterial vessels, with the most common sites being deep veins of the lower limbs and cerebral arteries (Keeling 2012). In people diagnosed with APS, about $13 \%$ have had a stroke and $7 \%$ a transient ischemic attack (TIA) (Panichpisal 2012), whereas aPL antibodies were found in about $20 \%$ of people under 50 years of age diagnosed with stroke (Bushnell 2000). It is well established that the simultaneous presence of all three types of antibodies ( $\mathrm{LA}, \mathrm{aCL}$, and anti$\beta_{2} \mathrm{GPI}$ ), the so called 'triple-antibody positivity,' is associated with a significantly higher thrombotic risk than the combination of two antibodies ('double-antibody positivity') or the presence of just one type of antibody ('single-antibody positivity') (Iwaniec 2016;
Pengo 2011; Pengo 2015). In a large cohort of unselected APS cases, the most frequently occurring clinical manifestations of APS were deep vein thrombosis, thrombocytopenia, livedo reticularis, and stroke, followed by pulmonary embolism, pregnancy loss, and transient ischemic attack (TIA) (Cervera 2002; Cervera 2009). TIA is defined as a condition with similar symptoms to a stroke, usually caused by a clot. However, the main difference between a stroke and TIA is that with TIA the blockage of the vessel is temporary and causes no permanent injury to the brain. According to the original definition, TIA symptoms should have resolved within 24 hours (Chatzikonstantinou 2013). According to the more recent definition - a tissue-based definition adopted by the American Heart Association and American Stroke Association (AHA/ASA) - TIA is defined as a transient episode of neurologic dysfunction, which is caused by focal ischemia of the nervous system tissue (brain, spinal cord, retinal), but with no acute infarction (Easton 2009).

Other clinical manifestations comprise heart valve disease, pre-eclampsia or eclampsia, premature birth, pulmonary hypertension, and leg ulcers (Ruiz-Irastorza 2010). The most severe form of APS is catastrophic APS (CAPS), which occurs in less than $1 \%$ of people with APS and has a mortality rate of 30\% (Cervera 2010).

Some studies have reported that people with arterial thrombosis are at higher risk of developing recurrence than those with venous thrombosis (Chighizola 2015). In a large European cohort study in which most participants had index venous thrombosis, recurrent arterial thromboses were the most common events (Cervera 2009). However, another study in people at high risk did not show that recurrent events depend on the index event (Chighizola 2015; Pengo 2010).

In a large cohort of unselected APS cases, the five-year survival rate in people with APS was approximately $90 \%$ to $94 \%$ (Cervera 2002; Cervera 2009). The median age at disease diagnosis was 31 years, and most people were diagnosed between the age of 15 and 50 years. Taking into account the clinical manifestations of the disease, which include cerebrovascular events $(13 \%$ of people with stroke and $7 \%$ of people with $\mathrm{TIA}$ ) and the young age of disease diagnosis onset, APS may exert a strong impact with high socioeconomic costs (Chighizola 2015).

Several studies have linked increased risk for thrombosis in people with aPL antibodies with the presence of cardiovascular risk factors such as hypercholesterolemia, smoking, and hypertension (Erkan 2007; Matyja-Bednarczyk 2014; Saraiva 2015). Therefore, careful assessment of the cardiovascular risk factors and their management in people with aPL is advised (Chighizola 2015).

\section{Description of the intervention}

There are no unconfounded randomized trials confirming the efficacy of either anticoagulants or antiplatelets compared with placebo or no treatment in the secondary prevention of thromboembolic events in APS. The thrombotic risk in definite APS is too high to allow using a placebo. Secondary thromboprophylaxis consists of antiplatelets (most commonly aspirin), anticoagulants (most commonly vitamin $\mathrm{K}$ antagonists (VKA): warfarin or acenocoumarol), or both (Espinosa 2015). People may receive aspirin or warfarin with the target international normalized ratio (INR) of 2.0 to 3.0 , or heparin when VKAs are contraindicated. Non-VKA antagonist oral anticoagulants (rivaroxaban, dabigatran, edoxaban, or apixaban) and antiplatelets 
(clopidogrel or prasugrel) can also be used alone or with aspirin. Where there are thrombotic complications at INR of 2.0 to 3.0, clinicians may modify the therapy to a higher target INR, combine two drugs, or, in high-risk patients, even prescribe triple antithrombotic therapy (one anticoagulant such as warfarin plus two antiplatelet agents, such as aspirin and clopidogrel). However, this is associated with a higher risk of hemorrhage or major bleeding (Nalli 2014). Selecting the appropriate intensity of anticoagulation to balance the risk of recurrent thrombosis and the risk of bleeding in APS is a real challenge for clinicians. Retrospective studies have suggested high-intensity (high-dose) anticoagulation with warfarin (INR set $>3$ ) to prevent recurrent thrombosis in people with APS (Lim 2006). However, randomized trials suggested adopting moderate-intensity anticoagulation with an INR targeted at 2.5 (range 2.0 to 3.0 ) as the best choice available for secondary thrombosis prevention in people with APS (Crowther 2003; Finazzi 2005). On the other hand, a randomized, doubleblind study testing the risk of subsequent thromboembolic events associated with positive aPL in patients with non-cardioembolic stroke failed to demonstrate the superiority of warfarin over aspirin therapy. Therefore, the study authors concluded that testing for aPL might not be warranted in all patients with ischemic stroke (APASS Investigators 2004).

Another important issue is the value of the INR at the time of recurrent thrombosis-studies have reported that in people who received VKA, most of the recurrent thrombotic events occurred in those with an INR below 3, while people in high-dose anticoagulation groups were below an INR range for over $40 \%$ of time, which may have biased the results (Chighizola 2015; RuizIrastorza 2011). This advice of moderate-intensity anticoagulation applied mainly to people with APS and venous thromboembolism; therefore, clinicians should exercise caution when adapting it to arterial thrombotic events, which are less frequent (Finazzi 2005; Ruiz-Irastorza 2011). It would be important to look at these two anticoagulant regimens as separate interventions. There are also studies suggesting that hydroxychloroquine can have an antithrombotic effect due to its antiplatelet properties (Erkan 2014; Ruiz-Irastorza 2011).

Intake of foods rich in vitamin $\mathrm{K}$, as well as drugs or other substances that may enhance or inhibit the metabolism of the anticoagulant agent used, can influence the effectiveness and safety of VKA (Chighizola 2014). VKA treatment often requires lifestyle modification and regular dose adjustment based on measured INR values, with monitoring for bleeding. These measures are necessary because they help to assure treatment effectiveness and safety. However, they may have a strong impact on the quality of life of the patient, since they would require regular visits to a healthcare facility: they can be time-consuming, may incur additional costs, and may interfere with daily activity to an extent that they are a considerable burden for some patients (Hasan 2015).

\section{How the intervention might work}

Aspirin is an antiplatelet agent, inhibiting cyclooxygenase 1 (COX-1) in platelets, which in turn inhibits the production of thromboxane A2 (TXA2) (Warner 2011). Clopidogrel is also an antiplatelet drug, but it works by inhibiting $\mathrm{P} 2 \mathrm{Y}$ receptors and impairs the activation of the glycoprotein (GP) Ilb/III complex by fibrinogen (Wijeyeratne 2011). The mechanism of action of oral anticoagulant agents such as warfarin or acenocoumarol is antagonizing vitamin $\mathrm{K}$ and thus inhibiting the production of coagulation factors II, VII, IX, X, and $C$ and S proteins (Ageno 2012). Heparin binds to antithrombin and then makes a complex with an activated factor $X$, inactivating it, which leads to the inhibition of blood coagulation (Hirsh 2001). Non-vitamin $\mathrm{K}$ antagonist oral anticoagulants (NOACs) are the direct inhibitors of either factor Ila (thrombin) or factor Xa (Weitz 2016). Dabigatran etexilate strongly and reversibly inhibits thrombin and thus restrains the conversion of fibrinogen into fibrin, while rivaroxaban and apixaban directly inhibit active factor $X$ (both free and thrombus-bound), which breaks the coagulation cascade (Ageno 2012).

The thrombotic risk in people with APS is high. Therefore, treatment guidelines currently recommend providing people with an APS diagnosis and thrombosis with life-long anticoagulants, antiplatelet therapy, or both, to prevent future arterial or venous thrombotic events (Ruiz-Irastorza 2011). However, these treatments increase the risk of bleeding, especially in people receiving the combination therapy (Nalli 2014), and they can also be associated with other adverse events (Raschi 2016). In the new guidelines for the diagnosis and management of acute pulmonary embolism developed by the European Society of Cardiology (ESC) in collaboration with the European Respiratory Society (ERC) the treatment with NOACs in patients with APS is not recommended (Konstantinides 2020). Similarly in the new recommendations for the management of APS in adults developed by the European League Against Rheumatism (EULAR) treatment with NOACs in high-risk APS patients and arterial events is not recommended due to higher risk of recurrent thrombosis (Tektonidou 2019a; Tektonidou 2019b).

\section{Why it is important to do this review}

No Cochrane Review has fully addressed the prevention of recurrent thrombosis in people with diagnosed APS. One recently published Cochrane Review focused on the effects of use of aspirin and/or heparin in women with persistent aPL antibodies and recurrent miscarriage (Hamulyak 2020), and a protocol registered by Cochrane Vascular considered using antiplatelet or anticoagulant agents to prevent recurrent peripheral vascular thrombosis in such patients (Islam 2016). A separate Cochrane Review focuses on primary prevention of thrombosis in people with aPL antibodies since they are different from those already diagnosed with APS (Bala 2018). However, none of these reviews addresses the issue of prevention of other types of thrombosis, such as stroke, in people with APS. Several randomized trials have examined the efficacy of using an antiplatelet drug (aspirin) or anticoagulant agents (such as warfarin) in people diagnosed with APS. A recent RCT of rivaroxaban versus warfarin in high-risk APS patients was prematurely terminated due to an increased risk of recurrent arterial thromboembolic events in the rivaroxaban arm (Pengo 2018). Some clinical trials using other NOACs in such patients are ongoing with a modified protocol (ASTROAPS). Therefore, it is important to summarize the effects of those therapies in people with APS while awaiting the results of the ongoing studies (ASTRO-APS; RISAPS).

\section{O B JECT IVES}

To assess the effects of antiplatelet or anticoagulant agents, or both, for the secondary prevention of recurrent thrombosis, particularly ischemic stroke, in people with APS. 


\section{METHODS}

\section{Criteria for considering studies for this review}

\section{Types of studies}

We included randomized controlled trials (RCTs) comparing participants allocated to one of two or more different treatment regimens. We also included RCTs comparing anticoagulant or antiplatelet treatments with placebo, usual care, or alternative treatment approaches.

\section{Types of participants}

People with APS, diagnosed according to the criteria valid when the study was carried out, such as the Sapporo or Sydney criteria (Miyakis 2006; Wilson 1999). We did not include studies specifically addressing women with recurrent miscarriages, as a separate Cochrane Review covers this topic (Hamulyak 2020).

\section{Types of interventions}

We included trials comparing any antiplatelet agents (e.g. aspirin, cilostazol, clopidogrel or prasugrel), any anticoagulant agents (e.g. VKA: acenocoumarol, warfarin; or NOAC: rivaroxaban, dabigatran, edoxaban, apixaban), or their combinations in any dose and mode of delivery versus no intervention/placebo or another antiplatelet/ anticoagulant regimen.

\section{Types of outcome measures}

\section{Primary outcomes}

- Any thromboembolic event, including death or any arterial or venous thrombosis

- Major bleeding

We defined major bleeding according to the International Society on Thrombosis and Haemostasis (ISTH) criteria, as clinically overt bleeding with a confirmed decrease in the hemoglobin level of at least $2 \mathrm{~g} / \mathrm{dL}$; or transfusion, due to the occurrence of clinical symptoms, of at least two units of packed red cells, occurring at a critical site (intracranial, intraocular, intraspinal, intraarticular, intramuscular with compartment syndrome, pericardial, retroperitoneal); or resulting in death (Schulman 2005). This definition does not consider any time restrictions.

\section{Secondary outcomes}

- Each type of thromboembolic event analyzed separately (i.e. allcause mortality, stroke, TIA, venous thromboembolism, etc)

- Quality of life measured with a validated questionnaire

- Any bleeding that does not meet the criteria for major bleeding

- Adverse event other than bleeding

We analyzed thromboembolic events as defined by the authors of the primary studies, especially with regard to TIA; this definition was originally time-based (Advisory Council 1975), but updates later based it on tissues (Easton 2009). If possible, we planned to take this into account in the subgroup analysis.

We assessed all outcomes at the end of follow-up.

\section{Search methods for identification of studies}

See the methods for the Cochrane Stroke Group Specialised register. We searched for trials in all languages and arranged for the translation or extraction of data of relevant articles where necessary.

\section{Electronic searches}

In addition to the Cochrane Stroke Group's trials register, we searched the following electronic databases (last search on 22 November 2019).

- Cochrane Central Register of Controlled Trials (CENTRAL; 2017, Issue 2)

- MEDLINE Ovid (from 1948)

- Embase Ovid (from 1980)

The search strategies for these electronic databases are presented in Appendix 1, Appendix 2, and Appendix 3. The subject strategies used for the included databases were based on the search strategy for MEDLINE designed by the Cochrane Stroke Group's Information Specialist (Appendix 2). These search strategies were combined with subject strategy adaptations of the highly-sensitive search strategy designed by Cochrane for identifying RCTs and controlled clinical trials, as described in the Cochrane Handbook for Systematic Reviews of Interventions Chapter 6.4.11 (Higgins 2011).

We also searched the following ongoing trials registries (last search on 22 November 2019).

- US National Institutes of Health trials registry, ClinicalTrials.gov (www.clinicaltrials.gov)

- Stroke Trials Registry (www.strokecenter.org/trials)

- European Trials Register (www.clinicaltrialsregister.eu)

- ISRCTN Registry (www.isrctn.com)

- The World Health Organization (WHO) International Clinical Trials Registry Platform (www.who.int/ictrp/en)

The search strategies for the trials registries are presented in Appendix 4.

\section{Searching other resources}

We checked reference lists of all included studies, systematic reviews, and practice guidelines relevant to the topic of the review. We contacted experts in the field and manufacturers of the original drugs to inquire about additional studies.

\section{Data collection and analysis}

\section{Selection of studies}

We used Covidence in the process of study selection. This software allows importing of search results, and facilitates independent screening by two review authors as well as comparing the results of screening, and extracting data from eligible studies. Pairs of review authors (MMB, MC-L, WS, AP, MK, MJS, and trainee reviewers named in the Acknowledgements) independently screened titles and abstracts of the references obtained as a result of our searching activities, and excluded obviously irrelevant reports. We retrieved the full-text articles for the remaining references, and pairs of review authors (MMB, MC-L, WS, AP, MK, MJS and trainee reviewers) 
independently screened them and identified studies for inclusion, as well as identifying and recording reasons for exclusion of the ineligible studies. We resolved any disagreements through discussion or, if required, we consulted a third review author (AU, MMB, WS, MC-L). We collated multiple reports of the same study so that each study, not each reference, was the unit of interest in the review. We recorded the selection process and completed a PRISMA flow diagram (Moher 2009).

\section{Data extraction and management}

We planned to use Covidence for data extraction, but the forms available in Covidence were not flexible enough to fit our extraction purposes; therefore, we decided to use Microsoft Excel 2013 spreadsheets. We extracted data on study settings, time frame and methods, population inclusion and exclusion criteria as well as population characteristics, details of interventions and cointerventions, and details of outcomes and their definitions. Pairs of review authors (MMB, MC-L, WS, AP, MK, MJS) independently extracted data from the included studies. We compared the extracted results and resolved any discrepancies by discussion. One review author (MMB) additionally checked all the data extracted.

\section{Assessment of risk of bias in included studies}

Pairs of review authors (MMB, MC-L, WS, AP, MK, MJS) independently assessed risk of bias for each study using the criteria outlined in the Cochrane Handbook for Systematic Reviews of Interventions chapter 8.5 (Higgins 2011). We resolved any disagreements by discussion or by involving another review author (MMB, MC-L, WS). We assessed the risk of bias according to the following domains.

- Random sequence generation

- Allocation concealment

- Blinding of participants and personnel

- Blinding of outcome assessment

- Incomplete outcome data

- Selective outcome reporting

- Other bias

We graded the risk of bias for each domain as high, low, or unclear and provided information from the study report together with a justification for our judgment in the 'Risk of bias' tables. We judged trials as being at low risk of bias if they were at low risk of bias in all of the domains; we judged other cases as being at high risk of bias.

\section{Measures of treatment effect}

For binary outcomes we calculated the risk ratio (RR) with 95\% confidence intervals (Cls); for continuous outcomes (quality of life) we planned to calculate the mean difference (MD) or standardized mean difference (SMD) (when different scales were used) with 95\% $\mathrm{Cls}$. Since only one study reported quality of life data, we presented the results as $\mathrm{MD}$ with $95 \% \mathrm{CI}$. For survival outcomes, such as hazard ratio (HR) for death, we used a generic inverse variance method for the meta-analysis. In all analyses we planned to calculate pooled estimates using the random-effects model (Der Simonian 1986), and we planned to conduct sensitivity analyses using the fixedeffect model meta-analyses (Greenland 1985; Mantel 1959). As we detected no heterogeneity in analyses where there was more than one study, we decided not to pursue the sensitivity analyses with the fixed-effect model.

\section{Unit of analysis issues}

Regarding unit of analysis issues, we planned to follow the advice of the Cochrane Handbook for Systematic Reviews of Interventions chapter 9.3 (Higgins 2011). We anticipated that in most trials the unit of analysis would be individual participants. However, if there were cluster-randomized trials, the unit of analysis would be the cluster. For cross-over trials, we would include the first phase only in the analysis. We did not include any cluster-randomized trials or cross-over trials in the review.

\section{Dealing with missing data}

If data were missing, we attempted to contact the study authors to request them. If unsuccessful, after examining the data set, we had two strategies: first, we assumed the data to be missing at random and we planned to analyze the data as reported. Second, if missing at random was not adequate, we assumed the data to be missing in a systematic way and we planned to assign all missing participants as treatment failures. Finally, we planned to conduct sensitivity analyses on missing data to test the impact of these approaches.

\section{Assessment of heterogeneity}

We used the $1^{2}$ statistic to measure heterogeneity among the trials in each analysis according to the Cochrane Handbook for Systematic Reviews of Interventions chapter 9.5.2 (Higgins 2011). We followed the interpretation in the Cochrane Handbook chapter 9.5.2 and considered $\mathrm{I}^{2}$ over $50 \%$ as representing substantial heterogeneity (Higgins 2011). In case of 12 over $50 \%$, we examined the characteristics of individual trials contributing to the comparison to identify potential sources of heterogeneity.

\section{Assessment of reporting biases}

We planned to analyze reporting bias using funnel plots for all primary outcomes if there were a sufficient number of studies (at least 10 studies as recommended in the Cochrane Handbook chapter 10.4 (Higgins 2011).

\section{Data synthesis}

Where we considered studies to be clinically, methodologically, and statistically similar, we conducted a meta-analysis by pooling the appropriate data using Review Manager (RevMan 2014). For binary outcome data we calculated RR or HR (if such data were available); for continuous outcomes (quality of life) we used MD. If necessary, we used the methods described by Parmar 1998 and Thierney 2007 to calculate data relevant to pool HR from the data available in the study (hazard rates, log rank $P$ values, events, ratios, curve data, follow-up information), using a spreadsheet in Microsoft Office Excel 2003 (freely available with the Thierney 2007 publication).

If pooling was not possible, we planned to summarize the results narratively, using text, figures, and tables.

\section{Subgroup analysis and investigation of heterogeneity}

If possible, we planned to explore heterogeneity by subgroup analyses taking into account single-, double- and triple-antibody positivity; lupus anticoagulant positivity versus other antibodies; presence versus absence of traditional cardiovascular risk factors; and type of index event (arterial versus venous).

We planned subgroup analyses for all outcomes only if there was a sufficient number of studies, i.e. for a single analysis at least 
six included studies with the information relevant for subgroup analysis (Deeks 2001).

\section{Sensitivity analysis}

We planned to conduct sensitivity analyses for missing data for each primary and secondary outcome for which they were missing, using worst-best, best-best, best-worst and worst-worst case scenarios. In addition, we planned to conduct sensitivity analyses according to low and high risk of bias and the amount of missing data (trials with no missing data versus trials with missing data).

\section{GRADE and 'Summary of findings' table}

We summarized the evidence in three 'Summary of findings' tables using GRADEpro (GRADEpro). In the development process, we followed the GRADE approach as outlined in the Cochrane Handbook for Systematic Reviews of Interventions chapter 11.5 (Higgins 2011). We planned to include the following outcomes in our 'Summary of findings' tables: any thromboembolic event, major bleeding, each type of thromboembolic event analyzed separately, quality of life, any bleeding that does not meet the criteria for major bleeding, and adverse events other than bleeding.

\section{RES ULT S \\ Description of studies}

See Characteristics of included studies; Characteristics of excluded studies; Characteristics of studies awaiting classification; Characteristics of ongoing studies.
None of the included studies compared antiplatelet or anticoagulant agent with placebo or no intervention; therefore, the effect of those agents compared to placebo or no intervention in secondary prevention of thromboembolic events in people with APS cannot be determined. The comparisons presented in the current review remain confounded because the interventions of unknown effect are compared with another intervention of unknown effect. No unequivocal evidence exists from randomized unconfounded studies that prove the efficacy of antiplatelets or anticoagulants in the secondary prevention of thromboembolic events in APS, but due to weaker evidence, these treatments are generally accepted and even included in recent clinical guidelines (Tektonidou 2019a; Tektonidou 2019b). Therefore, it is justified to perform the comparisons done in the current review.

\section{Results of the search}

We searched the electronic databases on 27 February 2017 and identified 12,180 references. We obtained an additional 13 records through searching other sources. Altogether, we screened 12,190 unique records by title and abstract after duplicates were removed (Figure 1). We downloaded full texts for 305 references. We disregarded 268 records because the study design $(n=219)$, population $(n=48)$, or intervention $(n=1)$ did not meet inclusion criteria. The remaining 37 references constituted five studies reported in 24 records (Crowther 2003; Okuma 2010; RAPS 2016; WAPS 2005; Yamazaki 2009); four ongoing trials, reported in eight references (ASTRO-APS; JASPRES; Ordi-Ros 2019; TRAPS 2016); and three studies awaiting classification, reported in five records (Kondratyeva 2010; Okuma 2014; Yamazaki 2007). 
Figure 1. Primary study flow diagram.

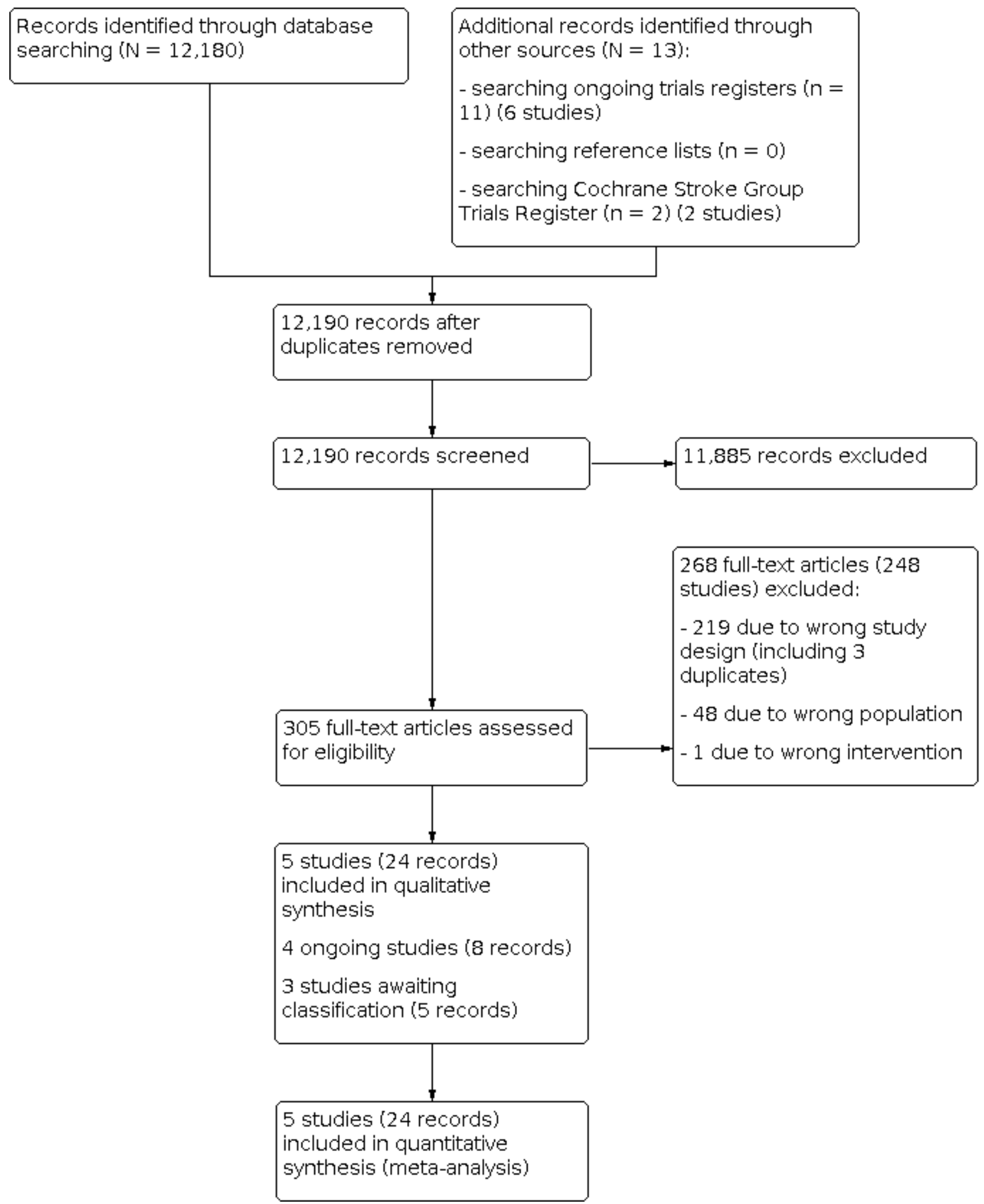

We updated our searches of the electronic databases and clinical trials registers on 22 November 2019, resulting in 459 records to screen. An additional two references were identified through searching other sources (Figure 2). Of 461 references, we excluded
448 based on title and abstract. Subsequently 13 records were checked in full text. We excluded five of them because of irrelevant study design $(n=4)$ and intervention $(n=1)$. 
Figure 2. Flow chart of identification of randomized trials for inclusion in the 22 November 2019 update. RCT: randomized clinical trial

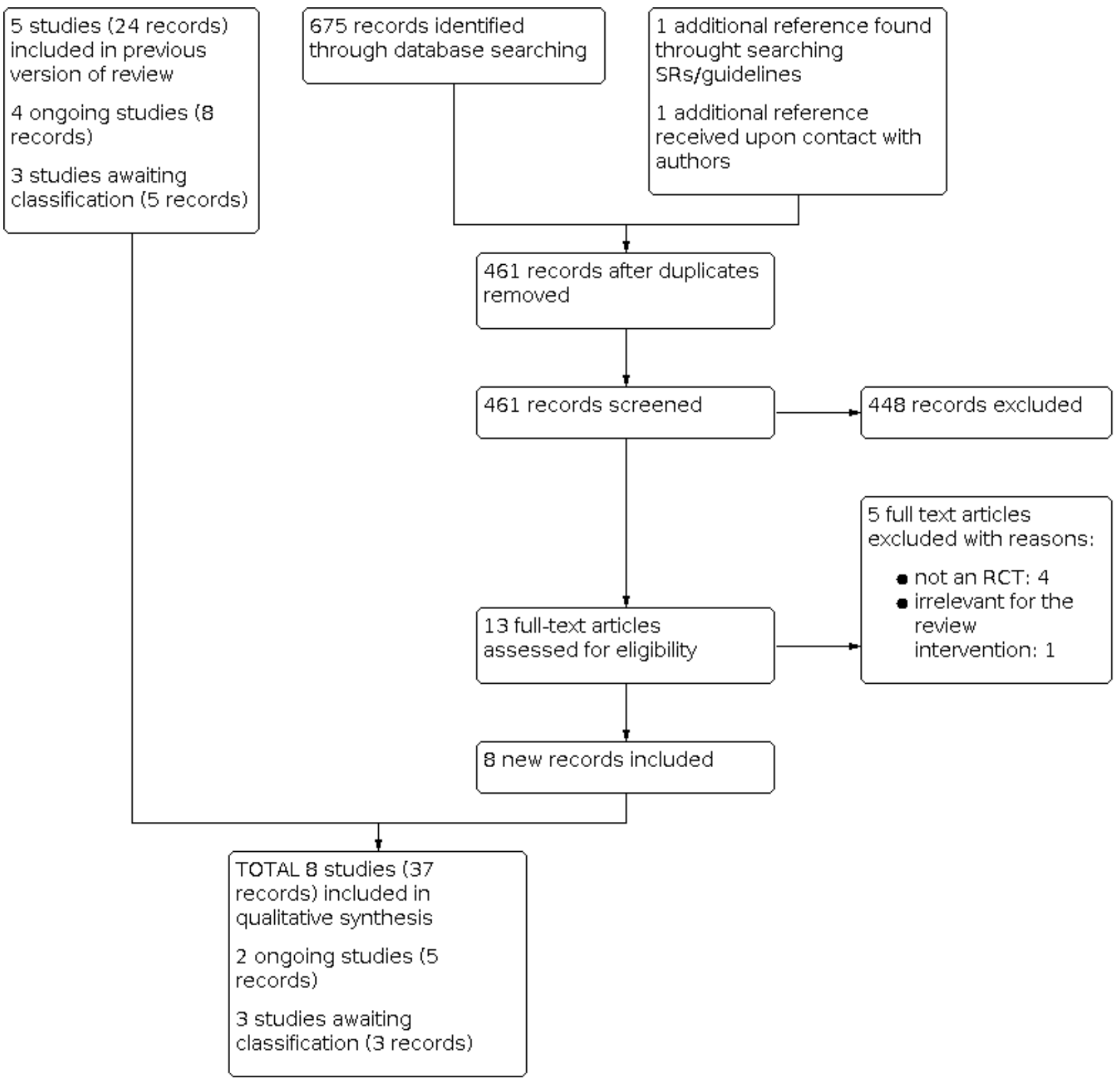

Eight new references were included in the review. Furthermore, we received additional information regarding the Kondratyeva 2010, TRAPS 2016, and Ordi-Ros 2019 studies, which resulted in the inclusion of these studies. We have not been able to obtain additional information on two of the awaiting assessment studies (Okuma 2014; Yamazaki 2007). As a result, in this update we included a total of eight studies reported in 37 records (Crowther 2003; Kondratyeva 2010; Okuma 2010; Ordi-Ros 2019; RAPS 2016; TRAPS 2016; WAPS 2005; Yamazaki 2009), two studies (five records) were listed as ongoing studies (ASTRO-APS; RISAPS), and three studies (three records) are awaiting classification (JASPRES; Okuma 2014; Yamazaki 2007).

\section{Included studies}

The study authors described the eight included studies as RCTs. Three were non-inferiority trials (Ordi-Ros 2019; RAPS 2016; TRAPS 2016), two were described as double blind (Crowther 2003; Okuma 2014), five were open label, but three had blinded endpoint adjudication for all outcomes (Ordi-Ros 2019; TRAPS 2016; WAPS 2005) or for safety events (RAPS 2016), and one study was published only in conference abstracts (Yamazaki 2009). We present detailed information on each study in Characteristics of included studies. 


\section{Participants}

All of the studies included people with diagnosed with APS. In total, 811 participants were randomized and 805 participants were analyzed for the outcomes relevant for this review.

The mean age of the participants in the study groups was 36.4 to 50 years; their median age was from 47 to 51 years. The criteria for inclusion of studies differed: Okuma 2010 specified and cited criteria for participants' diagnosis; Crowther 2003, Kondratyeva 2010 (information from authors), RAPS 2016, TRAPS 2016, and OrdiRos 2019 followed the criteria published in Miyakis 2006; WAPS 2005 used criteria published in Wilson 1999; and Yamazaki 2009 did not report specific inclusion criteria.

Yamazaki 2009 and Okuma 2010 included only people with previous stroke, while in RAPS 2016 a previous arterial event was an exclusion criterion, as was recurrent venous thromboembolism (VTE) while on warfarin with INR in the therapeutic range 2.0 to 3.0. Crowther 2003, Kondratyeva 2010, WAPS 2005, TRAPS 2016 , and Ordi-Ros 2019 included people with both previous arterial and VTE. However in those five studies the majority of participants had previous venous events (from $64 \%$ to $75 \%$ ).

Six studies reported the prevalence of SLE in study participants, which ranged from 13\% to 36\% (Crowther 2003; Okuma 2010; OrdiRos 2019; RAPS 2016; TRAPS 2016; WAPS 2005).

Five studies reported details about antibodies present (Crowther 2003; Ordi-Ros 2019; RAPS 2016; TRAPS 2016; WAPS 2005). Lupus anticoagulant was the only type of antibody detected for $43 \%$ of participants in Crowther 2003, 26\% in WAPS 2005, 46\% in RAPS 2016, and 35\% in Ordi-Ros 2019. Anticardiolipin antibodies were the sole type of antibody for $39 \%$ of participants in Crowther 2003, 18\% in WAPS 2005, and for 3\% of participants in RAPS 2016. Both types of antibodies were present for $18 \%$ of participants in Crowther 2003, and 56\% of the participants in WAPS 2005. RAPS 2016 also reported the percentage of participants with beta $_{2}$ glycoprotein I antibodies only (4\%) and more than one type of antibodies, both without (30\%) and including triple positivity (16\%). TRAPS 2016 included only participants with triple positivity for anticardiolipin, beta 2 glycoprotein I and lupus anticoagulant antibodies, while Ordi-Ros 2019 reported $61 \%$ of participants with IgG anticardiolipin and beta ${ }_{2}$ glycoprotein I antibodies, while $67 \%$ of participants with $\operatorname{lgG} / \operatorname{lgM}$ anticardiolipin antibodies, $64 \%$ with IgG/IgM beta 2 glycoprotein I antibodies and $34 \%$ with IgG/IgM antiphosphatidylserine/prothrombin antibodies.

Three studies reported on cardiovascular risk factors: Okuma 2010 reported hypertension (59.6\%), diabetes (20.2\%), hyperlipidemia (20.2\%), and atrial fibrillation (10.1\%); TRAPS 2016 reported smoking (50\%), hypertension (31\%), diabetes (3\%), dyslipidemia $(23 \%)$, and other conditions with increased tendency for coagulation (15\%); and Ordi-Ros 2019 reported smoking (35\%), dyslipidemia (39\%), diabetes mellitus (6\%), and hypertension (41\%).

\section{Location}

Yamazaki 2009 and Okuma 2010 took place in Japan, Crowther 2003 in Canada, Kondratyeva 2010 in Russia, Ordi-Ros 2019 in Spain, TRAPS 2016 in Italy, RAPS 2016 in the UK, and WAPS 2005 in Italy, Norway, Poland, Argentina, Czech Republic, and Slovak Republic.

\section{Setting}

Crowther 2003, WAPS 2005, Kondratyeva 2010, RAPS 2016, and TRAPS 2016 took place in specialist centres or clinics, Okuma 2010 in neurology departments of university hospitals, Ordi-Ros 2019 at university hospitals, and Yamazaki 2009 did not specify the setting.

\section{Interventions}

Three studies compared NOACs with standard-dose warfarin treatment (Ordi-Ros 2019; RAPS 2016; TRAPS 2016). RAPS 2016 compared a standard-dose warfarin treatment (mean INR 2.5) versus a NOAC: rivaroxaban $20 \mathrm{mg} / \mathrm{d}$. The mean INR in the warfarin group was 2.7 , and the mean time in therapeutic range at day 180 was $55 \%$. TRAPS 2016 compared rivaroxaban 20 or $15 \mathrm{mg} / \mathrm{d}$ (depending on renal function) with standard adjusteddose warfarin with a target INR between 2 and 3. Ordi-Ros 2019 compared rivaroxaban 20 or $15 \mathrm{mg} / \mathrm{d}$ (depending on renal function) with standard dose-adjusted VKA with INR 2 to 3 or 3.1 to 4 in case of a history of recurrent thrombosis. In the VKA group INR values were within the therapeutic range for a mean of $56 \%$ (median 58\% [IQR 46 to 70$])$.

Two studies compared treatment with two doses of warfarin: Crowther 2003 assessed high-dose warfarin with a target INR of 3.1 to 4.0 and an average value of 3.3 versus a standard dose with a target INR of 2.0 to 3.0 and an average value of 2.3 , and WAPS 2005 evaluated warfarin with a target of 3.5 and mean of 3.2 (range 3.0 to 4.5 ) versus standard antithrombotic therapy. Standard antithrombotic therapy included warfarin at a target 2.5 (range 2.0 to 3.0; mean 2.5) in participants with previous VTE, cardioembolic cerebral or peripheral ischemias, atrial fibrillation or rheumatic valve disease (95\% of participants), or aspirin 100 $\mathrm{mg} / \mathrm{d}$ in participants with non-embolic arterial thrombosis $(5 \%$ of participants). In addition, in Crowther 2003, 14\% of participants in the high-dose group and 10\% participants in the standard-dose group received aspirin, while in WAPS $20057 \%$ of participants in the high-dose group and $5 \%$ of participants in the standard-dose group received anticoagulation and aspirin according to the criteria of the treating physician. In Crowther 2003, participants in the highdose group were within the target INR for $40 \%$ of the time and below it for $43 \%$ of the time (but $86 \%$ of the time between 2.0 and 3.1 ), while in the standard-dose groups those values were $71 \%$ and $19 \%$, respectively.

Kondratyeva 2010 compared combined treatment with warfarin at a dosing regimen adjusted to a target INR of 2.0 to 3.0 and low-dose aspirin $(100 \mathrm{mg} / \mathrm{d})$ with treatment with warfarin alone.

Okuma 2010 and Yamazaki 2009 compared a single antiplatelet agent with combinations of antiplatelet and anticoagulant agents (VKA) or dual antiplatelet therapy. They included a comparison of aspirin $100 \mathrm{mg} / \mathrm{d}$ with a combination of aspirin and anticoagulant agents (a non-specified vitamin $\mathrm{K}$ antagonist) with a target INR of 2.0 to 3.0 (Okuma 2010), or a three-arm comparison of aspirin 100 $\mathrm{mg} / \mathrm{d}$, aspirin $100 \mathrm{mg} / \mathrm{d}$ plus cilostazol $200 \mathrm{mg} / \mathrm{d}$, and aspirin 100 $\mathrm{mg} / \mathrm{d}$ plus warfarin (with a target INR of 2.0 to 2.5 ) (Yamazaki 2009). The mean INR in the combined treatment group in Okuma 2010 was 2.4, while Yamazaki 2009 did not report these data.

The duration of intervention varied among the studies and ranged from 180 days to a median of 58.4 months (IQR 0.9 to 38.9). Only one study reported an additional 30 days follow-up without intervention (RAPS 2016). In Yamazaki 2009, one of the arms was 
stopped for "humanitarian" considerations (strokes revealed on MRI in three participants taking aspirin only). The TRAPS 2016 study was terminated prematurely due to excess thrombotic events in the rivaroxaban group.

\section{Outcomes}

The primary outcome in Yamazaki 2009 and Okuma 2010 was recurrent stroke. In Crowther 2003, recurrent thrombosis was the primary outcome. WAPS 2005 reported two co-primary outcomes: vascular death or major thrombosis (arterial or venous) and vascular death or major thrombosis or major hemorrhage. The primary outcome in RAPS 2016 was a surrogate outcome: percent change in endogenous thrombin potential from randomization to day 42 of study, plus reported thromboembolism (VTE or any other thrombotic events) up to day 210 as a secondary outcome. Kondratyeva 2010 studied frequency of recurrent thromboses and TIA. The primary outcome in Ordi-Ros 2019 was new thrombotic event, while TRAPS 2016 specified composite primary outcome including thromboembolic events, major bleeding, and vascular death.

In five trials, safety outcomes included major, minor, or any bleeding (Crowther 2003; Okuma 2010; Ordi-Ros 2019; RAPS 2016; WAPS 2005), one study reported only on major bleeding (TRAPS
2016), and one study on major and minor bleeding (Kondratyeva 2010).

Study authors did not specify the secondary outcomes in Crowther 2003, Kondratyeva 2010, Yamazaki 2009 and Okuma 2010. OrdiRos 2019 specified as secondary outcomes the time to thrombosis, type of thrombotic event, death from cardiovascular causes, and changes in selected biomarkers' levels. The secondary outcomes for efficacy in RAPS 2016 included other coagulation measures and quality of life (measured using the EQ-5D-5L questionnaire). TRAPS 2016 specified as secondary outcomes type of thrombotic events and mortality from all causes. The secondary endpoints in WAPS 2005 included combinations of different thrombotic events. Two trials specifically reported adverse events as outcomes (RAPS 2016; WAPS 2005).

\section{Excluded studies}

We excluded one study because it was irrelevant for the review population; see Characteristics of excluded studies.

\section{Risk of bias in included studies}

We presented details for each study in the Characteristics of included studies table. Figure 3 shows the overall risk of bias in each domain for studies in this review; Figure 4 shows risk of bias by trial.

Figure 3. Risk of bias graph: review authors' judgements about each risk of bias item presented as percentages across all included studies. Duplicate blinding domains are due to separate assessments for objective or objectively verified outcomes (Obj.) and subjective and self-reported outcomes (Subj.). Blank sections are left because those studies did not assess subjective outcomes or patient self-reported outcomes not objectively verified.

Random sequence generation (selection bias) Allocation concealment (selection bias) Blinding of participants and personnel (performance bias): Obj. Blinding of participants and personnel (performance bias): Subj. Blinding of outcome assessment (detection bias): Obj. Blinding of outcome assessment (detection bias): Subj. Incomplete outcome data (attrition bias): All outcomes Selective reporting (reporting bias)

Other bias
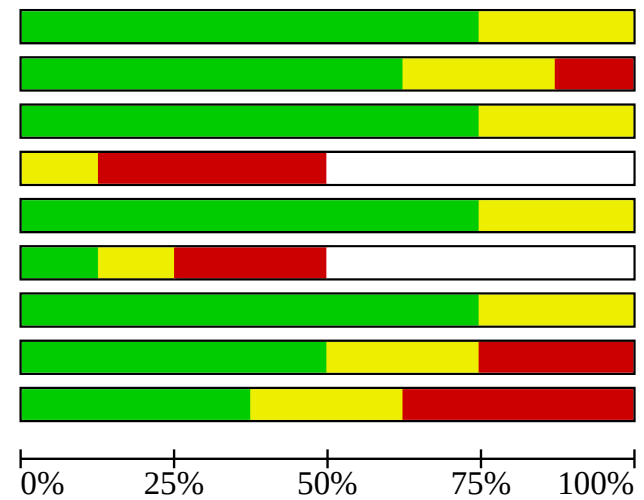
Figure 4. Risk of bias summary: review authors' judgements about each risk of bias item for each included study. Duplicate blinding domains are due to separate assessments for objective or objectively verified outcomes (Obj.) and subjective and self-reported outcomes (Subj.). Blank sections are left because those studies did not assess subjective outcomes or patient self-reported outcomes not objectively verified.

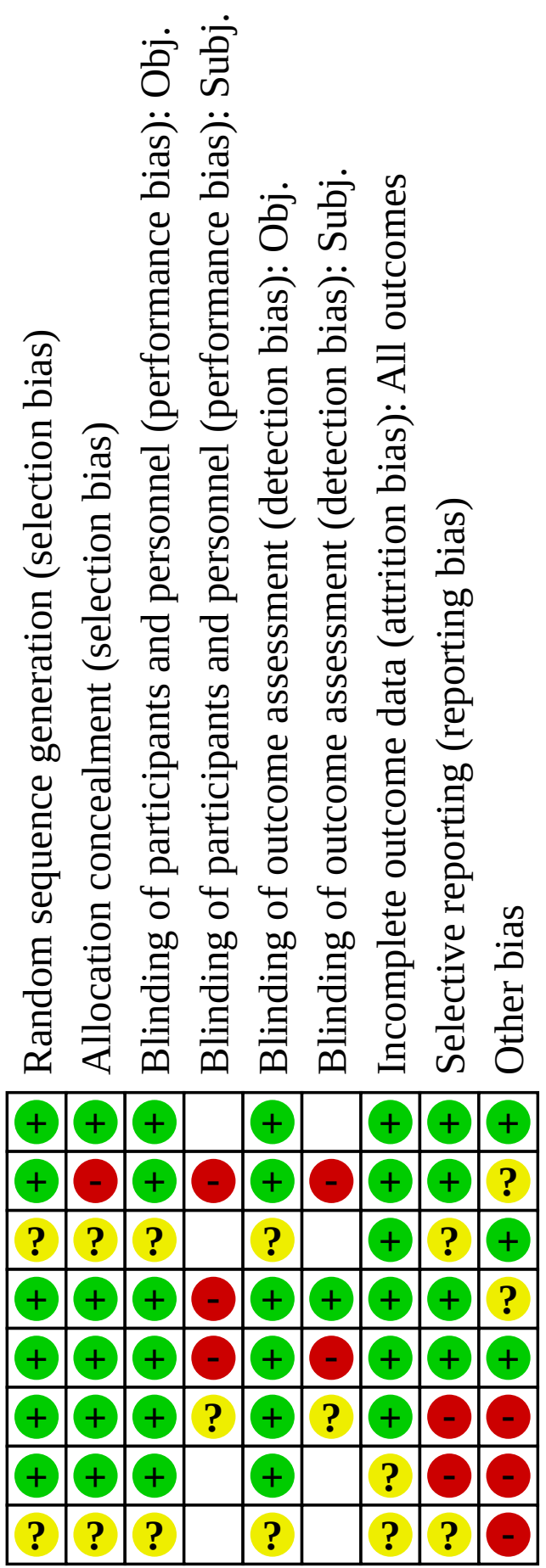


Seven of the included studies were published as full articles. One consisted of several conference abstracts, so data extracted from this study were very limited (Yamazaki 2009).

Only one study was at low risk of bias in all domains (Crowther 2003). One study was at low risk of bias in all domains for objective outcomes, but not for subjective ones (RAPS 2016).

\section{Allocation}

In two studies, the risk of bias in random sequence generation and allocation concealment domains was unclear, as no detailed information was provided (Okuma 2010; Yamazaki 2009).

Kondratyeva 2010 used coin tossing for treatment assignment without allocation concealment; we therefore judged it to be low risk of bias for sequence generation and at high risk of bias for allocation concealment.

The five other studies presented clear information, and we judged them to be at low risk of bias in these domains (Crowther 2003; Ordi-Ros 2019; RAPS 2016; TRAPS 2016; WAPS 2005). Studies used central randomization or sequentially-numbered sealed envelopes: sequence generation was ensured by means of a random numbers table (Crowther 2003), computer-generated random number sequences in blocks of 10 (Ordi-Ros 2019), random permuted blocks of various length with stratification by centre and patient type (RAPS 2016), with the use of web-based management system with random blocks and stratified (TRAPS 2016), and a program based on the biased-coin algorithm (WAPS 2005).

\section{Blinding}

We assessed blinding of participants, personnel, and outcome assessors for two groups of outcomes: objective outcomes, for example stroke, bleeding, and mortality; and subjective outcomes, such as quality of life or self-reported outcomes (adverse events, minor bleeding).

Yamazaki 2009 did not provide any information about blinding, so the risk of bias in this study was unclear. Okuma 2010, although described as double-blinded, did not provide clear information about blinding; it also did not provide clear information about outcome definition or verification, so we judged the risk of bias as unclear.

Crowther 2003 explicitly stated that the trial was double-blinded and that the participants, treating physicians, auxiliary personnel, and a panel of outcomes assessors were all unaware of the treatment assignments; we considered the risk of bias in this study to be low. WAPS 2005, Kondratyeva 2010, RAPS 2016, and Ordi-Ros 2019 did not blind the participants or personnel. In our judgement, due to the objective definition or verification of outcomes in those studies, the lack of blinding likely did not influence objective outcomes, so we judged them to be at low risk of bias for those outcomes, but the lack of blinding could influence subjective outcomes, such as quality of life in RAPS 2016 or only self-reported outcomes (Kondratyeva 2010; Ordi-Ros 2019), so we judged it to be at a high risk of bias for subjective or not objectively verified outcomes. Ordi-Ros 2019 also reported the independent committee which reviewed all adverse events (also those not specified as objectively verified) therefore we judged blinding of outcome assessors to be at low risk of bias for this study.
WAPS 2005 explicitly stated that outcome assessors were blinded, so we judged the risk of bias to be low. TRAPS 2016 did not blind participants and personnel, but reported blinded adjudication for primary outcomes and its composites, so it was judged to be low risk of bias for those outcomes but it did not report information about outcome assessors for adverse events so it was judged to be unclear for subjective outcomes.

\section{Incomplete outcome data}

We assessed Crowther 2003, Kondratyeva 2010, Okuma 2010, RAPS 2016, TRAPS 2016, and Ordi-Ros 2019 to be at low risk of bias for incomplete outcome data. Reasons included little or no missing data, trials applied the intention-to-treat (ITT) principle, missing data were balanced between the groups, or the reasons for missing data were reported and were unlikely to be related to study outcomes. Although WAPS 2005 reported results for all randomized participants, it did not follow up 6/109 participants (information confirmed with the study authors; it is not clear from which group), and although the number of participants in the analysis equaled the number of participants randomized, it is not clear how the participants who were not followed up were included in the analysis. In Yamazaki 2009, there was insufficient information available, so we judged the risk of bias as unclear.

\section{Selective reporting}

We assessed the risk of bias for selective reporting in Crowther 2003, Kondratyeva 2010, RAPS 2016, and Ordi-Ros 2019 as low. RAPS 2016 provided a protocol and reported outcomes as specified in the protocol. We did not identify the protocol for Crowther 2003 or for Kondratyeva 2010 but judging from the texts of the articles it was clear that the published reports include all expected outcomes, including those that were pre-specified in the methods section.

TRAPS 2016 had published a protocol in which it also indicated minor bleeding and adverse events but in the short report from the study it did not report those findings. As a result, we judged this trial to be at high risk of bias for selective reporting.

We obtained a protocol for WAPS 2005, but there were several discrepancies between the outcomes listed there and in the study publication, so we judged the risk of bias to be high.

We did not identify the protocol for Okuma 2010, and the primary outcomes were partially reported as indicated in the methods section; but the numbers of events were not reported, so we judged the risk of bias to be unclear.

We did not identify the protocol for Yamazaki 2009, and the information provided was insufficient to judge the risk of bias.

\section{Other potential sources of bias}

We did not identify any source of other potential bias in three studies (Crowther 2003; Okuma 2010; RAPS 2016). WAPS 2005 was seriously underpowered: the planned sample size was 500 participants per arm, while the number of participants recruited to the study was 109 in total and the study was terminated early due to poor recruitment. With respect to the early termination of the trial, we assessed risk of bias as high for this domain. TRAPS 2016 planned to enroll 536 participants; however, the trial was terminated early after including 120 participants. Therefore, we judged this study to be at high risk of bias for this domain. In 
Yamazaki 2009 all three groups were planned to be followed up for three years; however, in the group treated with aspirin alone the intervention was discontinued after a year for "humanitarian" reasons, thus we judged this study to be at high risk of bias for this domain. Kondratyeva 2010 and Ordi-Ros 2019 did not provide sufficient information to judge any other potential sources of bias.

\section{Effects of interventions}

See: Summary of findings 1 NOAC (rivaroxaban) versus standarddose VKA; Summary of findings 2 High-dose VKA versus standarddose VKA; Summary of findings 3 Standard-dose VKA plus single antiplatelet agent versus standard-dose VKA; Summary of findings 4. Standard-dose VKA plus single antiplatelet agent versus single antiplatelet agent; Summary of findings 5 Standard-dose VKA plus single antiplatelet agent versus dual antiplatelet agent; Summary of findings 6 Dual antiplatelet agent versus single antiplatelet agent

The studies contributed data regarding six separate comparisons:

- NOAC (rivaroxaban) versus standard-dose VKA;

- high-dose VKA versus standard-dose VKA;

- standard-dose VKA plus single antiplatelet agent versus standard-dose VKA;

- standard-dose VKA plus single antiplatelet agent versus single antiplatelet agent;

- standard-dose VKA plus single antiplatelet agent versus dual antiplatelet agent;

- dual antiplatelet agent versus single antiplatelet agent

We reported the results for each outcome within these comparisons, and provided separate 'Summary of findings' tables (Summary of findings 1; Summary of findings 2; Summary of findings 3; Summary of findings 4; Summary of findings 5 ; Summary of findings 6 ).

The comparisons presented in this review are based on the assumption (and not on unconfounded randomized evidence) that usual treatment with VKA (INR 2.0-3.0) is effective in reducing the recurrence of thromboembolic events in patients with APS. For the last comparison (dual antiplatelet versus single antiplatelet) the assumption is, that a single antiplatelet is effective in reducing recurrence of thromboembolic events in patients with APS.

With respect to missing data, RAPS 2016 excluded participants' data from the analysis only in the control group (one participant for the analysis of thrombotic events and four participants for the analysis of bleeding events) but not in the experimental group, so we tested worst-case (assuming that all participants receiving warfarin who had missing data had the best possible outcome) and best-case (assuming that all participants receiving warfarin who had missing data had the worst possible outcome) scenarios for the missing data of participants in the control group. WAPS 2005 did not provide information about the treatment arm of participants who were not followed up, therefore we could not attempt any sensitivity analysis.

Due to low number of studies for each comparison and outcome, we did not produce funnel plots.

We did not detect significant heterogeneity in any of the comparisons; however, we report results separately for different comparisons separately for anticoagulants, antiplatelets, and different sets of their combinations. We did not attempt any subgroup analyses because of the limited number of studies included in the review.

In the 'Summary of findings' tables, due to the limitations in the number of outcomes reported, we only included stroke and death for 'each type of thromboembolic event analyzed separately.'

\section{NOAC (rivaroxaban) versus standard-dose VKA}

\subsection{Any thromboembolic event}

Three studies were included in the analysis (Ordi-Ros 2019; RAPS 2016; TRAPS 2016); however, RAPS 2016 did not report any events of recurrent VTE or other thrombotic event at 210 days of follow-up; it was also not powered to detect differences in the occurrence of clinical events. TRAPS 2016 did not report occurrence of any events in the standard dose VKA (warfarin) group. Overall total number of events was 20 in the NOAC (rivaroxaban) group and six in the standard-dose VKA group. Meta-analysis of the results provided an RR of 4.08 (95\% Cl 0.48 to $34.79 ; 425$ participants; three studies; $\left.\right|^{2}=$ 57\%; moderate-certainty evidence; Analysis 1.1). The heterogeneity observed in this comparison may be partially explained by the difference in the population, i.e. in the TRAPS 2016 study only patients with high risk (triple-positivity), while in Ordi-Ros 2019 such high-risk patients comprised $60 \%$ of the whole population.

The sensitivity analysis did not change the statistical significance of the results (Analysis 7.1; Analysis 7.2).

\subsection{Major bleeding}

Three studies were included in the analysis (Ordi-Ros 2019; RAPS 2016; TRAPS 2016); however, RAPS 2016 did not report any events of major bleeding at 210 days of follow-up; it was also not powered to detect differences in the occurrence of clinical events. Overall total number of events was 10 in the NOAC (rivaroxaban) group and nine in the standard-dose VKA group. Meta-analysis of the results provided an RR of 1.10 ( $95 \% \mathrm{Cl} 0.45$ to 2.68 ; 425 participants; three studies; $1^{2}=0 \%$; moderate-certainty evidence; Analysis 1.2). Statistical significance of the results did not change when we pooled the log HR, calculated on the basis of data reported in the studies (Analysis 1.3).

The sensitivity analysis did not change the statistical significance of the results (Analysis 7.3; Analysis 7.4).

\subsection{All-cause mortality}

Three studies were included in this analysis (Ordi-Ros 2019; RAPS 2016; TRAPS 2016). Total number of deaths was six in the NOAC (rivaroxaban) group and four in the standard-dose VKA group. RAPS 2016 reported one death due to non-Hodgkin's lymphoma in a participant taking warfarin during 210 days of follow-up, TRAPS 2016 reported one death due to cardiovascular causes in the VKA group, while Ordi-Ros 2019 reported eight deaths due to cancer, septic shock, pulmonary hemorrhage, pulmonary hypertension, and cardiac failure. Meta-analysis of the results provided an RR of 1.45 (95\% Cl 0.44 to 4.78 ; 425 participants; three studies; $\left.\right|^{2}=0 \%$; moderate-certainty evidence; Analysis 1.4).

The sensitivity analysis did not change the statistical significance of the results (Analysis 7.5; Analysis 7.6). 


\subsection{Stroke}

Three studies were included in this analysis (Ordi-Ros 2019; RAPS 2016; TRAPS 2016). The total number of events was 14 in the NOAC (rivaroxaban) group and no cases in the standard dose VKA group. RAPS 2016 did not report any events in the NOAC (rivaroxaban) or the warfarin standard dose groups at 210 days of follow-up, but the trial was not powered to detect differences in the occurrence of clinical events. TRAPS 2016 reported four participants with stroke in the NOAC (rivaroxaban) group, while Ordi-Ros 2019 reported 10 participants with stroke in the NOAC (rivaroxaban) group. Metaanalysis of the results indicate an increased risk of stroke with NOAC (rivaroxaban) treatment with an RR of $14.13(95 \% \mathrm{Cl} 1.87$ to 106.81; 425 participants; three studies; $\left.\right|^{2}=0 \%$; moderate-certainty evidence; Analysis 1.5).

\subsection{Transient ischemic attack}

Three studies were included in this analysis (Ordi-Ros 2019; RAPS 2016; TRAPS 2016). None of them reported any TIA events during follow-up (Analysis 1.6).

\subsection{Venous thromboembolism (VTE)}

Three studies were included in this analysis (Ordi-Ros 2019; RAPS 2016; TRAPS 2016). RAPS 2016 did not report any events at 210 days of follow-up, but it was not powered to detect differences in the occurrence of clinical events. TRAPS 2016 reported one participant with VTE in the NOAC (rivaroxaban) group, and Ordi-Ros 2019 reported two cases of VTE in the NOAC (rivaroxaban) group and three in the standard dose VKA group. Meta-analysis of the results provided an RR of 0.96 ( $95 \% \mathrm{Cl} 0.20$ to 4.49; 425 participants; three studies; $\left.\right|^{2}=0 \%$; Analysis 1.7 ).

\subsection{Myocardial infarction (MI)}

Three studies were included in this analysis (Ordi-Ros 2019; RAPS 2016; TRAPS 2016). Similar to other endpoints, RAPS 2016 did not report any events at 210 days of follow-up. TRAPS 2016 reported three participants with $\mathrm{MI}$ in the NOAC (rivaroxaban) group, and Ordi-Ros 2019 reported no cases of MI in both groups.

Meta-analysis of the results provided an RR of $7.23(95 \% \mathrm{Cl} 0.38$ to 137.08; 425 participants; three studies; $I^{2}=0 \%$; Analysis 1.8 ).

\subsection{Other thrombotic events}

Three studies were included in this analysis (Ordi-Ros 2019; RAPS 2016; TRAPS 2016). RAPS 2016 reported no cases of microvascular thrombosis at 210 days of follow-up (Analysis 1.9). TRAPS 2016 reported no cases of other arterial thrombotic events, and OrdiRos 2019 reported two arterial events other than MI and stroke in the NOAC (rivaroxaban) group and three in the standard dose VKA group (Analysis 1.9). The RR for the other arterial thrombotic events, based on Ordi-Ros 2019 and no events in TRAPS 2016, was 0.67 (95\% Cl 0.11 to $3.90 ; 310$ participants; two studies; $\left.\right|^{2}=$ not applicable; Analysis 1.9)

\subsection{Quality of life measured with a validated questionnaire}

Only one study reported on this outcome (RAPS 2016). The study reported the mean results of the EQ-5D-5L questionnaire at day 180 in terms of health utility. The calculated MD was $0.04(95 \% \mathrm{Cl}-0.02$ to 0.10 on a scale from 0 to 1 ; 111 participants; one study; lowcertainty evidence; Analysis 1.10).
Regarding the Health State Visual Analogue Scale, the results showed a statistically significant difference in favor of NOAC (rivaroxaban) with MD $7 \mathrm{~mm}$ (95\% Cl 2.01 to 11.99 ; 112 participants; one study; low-certainty evidence; Analysis 1.10).

\subsection{Any bleeding that does not meet the criteria for major bleeding}

Two studies were included in this analysis (Ordi-Ros 2019; RAPS 2016). RAPS 2016 reported three participants with clinically relevant non-major bleeding in the NOAC (rivaroxaban) group and two participants in the standard dose VKA group. Ordi-Ros 2019 reported nine participants with clinically relevant non-major bleeding in the NOAC (rivaroxaban) group and five in the standard dose VKA group. Meta-analysis of the results provided an RR of 1.70 (95\% Cl 0.69 to 4.19; 302 participants; two studies; $\left.\right|^{2}=0 \%$; moderate-certainty evidence; Analysis 1.11).

RAPS 2016 reported 10 participants with minor bleeding in the NOAC (rivaroxaban) group and eight participants in the standard dose VKA group. Ordi-Ros 2019 reported 16 participants with minor bleeding in the NOAC (rivaroxaban) group and 14 in the standard dose VKA group. Meta-analysis of the results provided an RR of 1.17, $95 \% \mathrm{Cl} 0.69$ to $1.96 ; 302$ participants; two studies; $1^{2}=0 \%$; Analysis $1.12)$.

As four participants from the warfarin group were excluded from the analysis in the RAPS 2016 study, we performed a sensitivity analysis. It did not change the statistical significance of the results (Analysis 7.7; Analysis 7.8; Analysis 7.9; Analysis 7.10).

\subsection{Adverse events other than bleeding}

Ordi-Ros 2019 reported similar numbers of participants with any adverse events during treatment $(62.1 \%$ versus $58.9 \%$; RR 1.05 , $95 \% \mathrm{Cl} 0.84$ to $1.3 ; 190$ participants; calculated using RevMan calculator). Eleven participants in the NOAC (rivaroxaban) group and six participants in the standard dose VKA group experienced adverse events which led to withdrawal from the study. There were eight cases of serious adverse events in each group, which occurred between the date when first dose was administered up to 30 days after last visit. In the NOAC (rivaroxaban) group they included events leading to death, such as malignant conditions (three cases), urinary septic shock and pulmonary hemorrhage due to capillaritis (one case each), and not leading to death, such as flare of SLE (two cases) and pneumonia (one case). In the VKA group serious adverse events included one case of malignant condition leading to death, and single cases of cardiac failure, pulmonary hypertension, endocarditis, soft tissue infection, SLE flare, cholecystitis, and intestinal perforation.

RAPS 2016 reported the occurrence of serious adverse events in four participants receiving NOAC (rivaroxaban) and four participants receiving standard dose VKA (warfarin). In the NOAC (rivaroxaban) group, investigators judged two of those events to be unrelated to the study drug: one was a previous intracranial hemorrhage, incidentally detected on brain imaging without any new or recurrent symptoms, and the other was a grade 1 event (grade 2 abdominal pain, vomiting, arthralgia, and myalgia). Additionally, two events were judged unlikely to be related to the study drug (grade 4 intestinal perforation; grade 2 suspected deep vein thrombosis on the basis of a Doppler ultrasound judged to be related to previous femoral vein deep vein thrombosis and without any new thrombosis). In the standard dose VKA (warfarin) group, 
investigators judged three events to be unrelated to the study drug (grade 3 asthma exacerbation; grade 4 sepsis; high-grade nonHodgkin's lymphoma stage IV B, which resulted in death), and they classified one event as a grade 3 serious adverse reaction, probably related to standard dose VKA (warfarin) treatment (hemorrhoidal hemorrhage).

TRAPS 2016 did not report on adverse events other than bleeding.

\section{High dose VKA versus standard dose VKA}

\subsection{Any thromboembolic event}

Two studies compared the effects of high and standard doses of VKA (warfarin): Crowther 2003 assessed the recurrence of any thrombosis, and WAPS 2005 vascular death or major thrombosis. Together, they reported a total of 11 versus five events during a mean of 2.7 years (SD not reported) and a mean of 3.4 years (SD 1.2) of follow-up respectively. Meta-analysis of the results provided an RR of 2.22 (95\% Cl 0.79 to $6.23 ; 223$ participants; two studies; $\left.\right|^{2}=$ $0 \%$; low-certainty evidence; Analysis 2.1). Statistical significance of the results did not change when we pooled the log HR, calculated on the basis of data reported in the studies (Analysis 2.2).

Crowther 2003 reported INR values in the two participants with thrombotic events in the standard dose group (INR 1.6 and INR 2.8), while the INR values were $3.1,1.0,0.9,1.9$, and 3.9 in five out of six participants with events in the high-dose group (one participant discontinued treatment).

\subsection{Major bleeding}

Two studies comparing high and standard dose VKA (warfarin) examined major bleeding (Crowther 2003; WAPS 2005), reporting five versus seven cases in total during a mean of 2.7 years (SD not reported) and a mean of 3.4 years (SD 1.2) of follow-up, respectively. Meta-analysis of the results provided an RR of $0.74(95 \% \mathrm{Cl} 0.24$ to 2.25 ; 223 participants; two studies; $1^{2}=0 \%$; low-certainty evidence; Analysis 2.3). Statistical significance of the results did not change when we pooled the log HR, calculated on the basis of data reported in the studies (Analysis 2.4).

\subsection{All-cause mortality}

Two studies comparing high and standard dose VKA (warfarin) reported either no death during a mean of 2.7 years (SD not reported) of follow-up (Crowther 2003), or three versus two deaths during a mean of 3.4 years (SD 1.2) years of follow-up (WAPS 2005). Meta-analysis of the results provided an RR of $1.53(95 \% \mathrm{Cl} 0.27$ to 8.79; 223 participants; two studies; $12=0 \%$; low-certainty evidence; Analysis 2.5).

\subsection{Stroke}

Two studies assessed the effect of high-dose versus standard-dose VKA (warfarin) (Crowther 2003; WAPS 2005). The number of events was low (three versus two events) during a mean of 2.7 years (SD not reported) and a mean of 3.4 years (SD 1.2) of follow-up, respectively. Meta-analysis of the results provided an RR of $1.37(95 \% \mathrm{Cl} 0.26$ to 7.12; 223 participants; two studies; $12=0 \%$; low-certainty evidence; Analysis 2.6).

\subsection{Transient ischemic attack}

Crowther 2003 and WAPS 2005 both used the TIA definition based on the time of symptoms occurrence. Crowther 2003 reported no
TIA events over a mean of 2.7 years (SD not reported) of follow-up, while in WAPS 2005 the number of events was low in both the highand standard dose warfarin groups (two versus one event) during a mean of 3.4 years (SD 1.2) of follow-up. Meta-analysis of the results provided an RR of 2.04 ( $95 \% \mathrm{Cl} 0.19$ to $21.81 ; 223$ participants; two studies; $1^{2}=0 \%$; Analysis 2.7).

\subsection{Venous thromboembolism (VTE)}

In two studies comparing the effect of high-dose versus standarddose VKA (warfarin), the number of events was low (six versus one event) during a mean of 2.7 years (SD not reported) and a mean of 3.4 years (SD 1.2) of follow-up, respectively (Crowther 2003; WAPS 2005). Meta-analysis of the results provided an RR of $4.44(95 \% \mathrm{Cl}$ 0.77 to 25.72 ; 223 participants; two studies; $\left.\right|^{2}=0 \%$; Analysis 2.8 ).

\subsection{Myocardial infarction}

From two studies comparing high-dose warfarin with standarddose VKA (warfarin), WAPS 2005 did not report any event at a mean of 3.4 years (SD 1.2) of follow-up, while Crowther 2003 reported a single event in each of the treatment groups during a mean of 2.7 years (SD not reported) of follow-up. Meta-analysis of the results provided an RR of 1.04 ( $95 \% \mathrm{Cl} 0.07$ to $16.16 ; 223$ participants; two studies; $\left.\right|^{2}=0 \%$; Analysis 2.9).

\subsection{Other thrombotic events}

WAPS 2005 reported a single case of superficial vein thrombosis in the high-dose group over a mean of 3.4 years (SD 1.2) of followup, resulting in the calculated RR of 3.05 ( $95 \% \mathrm{Cl} 0.13$ to $73.37 ; 109$ participants; one study; Analysis 2.10).

\subsection{Any bleeding that does not meet the criteria for major bleeding}

In WAPS 2005 the occurrence of minor bleeding was more frequent in the high-dose warfarin group compared with the standard-dose warfarin group during a mean of 3.4 years (SD 1.2) of follow-up (RR $2.55,95 \% \mathrm{Cl} 1.07$ to 6.07 ; 109 participants; one study; Analysis 2.11). The authors of the WAPS 2005 study also reported minor bleeding as a HR, showing a higher rate of those events with a higher dose (HR 2.92, 95\% Cl 1.13 to 7.52; 109 participants; one study).

Two studies comparing high-dose and standard-dose VKA (warfarin) reported on any bleeding during a mean of 2.7 years (SD not reported) and a mean of 3.4 years (SD 1.2) of followup, respectively (Crowther 2003; WAPS 2005). Meta-analysis of the results provided an RR of $1.56(95 \% \mathrm{Cl} 0.93$ to $2.62 ; 223$ participants; two studies; $12=0 \%$; low-certainty evidence; Analysis 2.12). However, when we pooled log HRs, calculated using the data reported in the published studies, the difference became significant, indicating a higher risk of any bleeding in the high-dose warfarin group (HR 2.03, 95\% Cl 1.12 to 3.68; Analysis 2.13).

\subsection{Adverse events other than bleeding}

WAPS 2005 reported on any adverse events leading to treatment withdrawal and reported two withdrawals associated with reported events, such as essential thrombocythemia in one participant and headache in one participant, but the study authors did not indicate the group in which those participants were included.

Antiplatelet and anticoagulant agents for secondary prevention of stroke and other thromboembolic events in people with 
3 Standard dose VKA plus single antiplatelet agent versus standard dose VKA

\subsection{Any thromboembolic event}

Kondratyeva 2010 reported on recurrent thrombosis and recurrent TIA and the combined numbers of participants with those events were 13 in the standard-dose VKA plus antiplatelet agent group and nine in the standard-dose VKA group, resulting in an RR of 2.14 (95\% $\mathrm{Cl} 1.04$ to 4.43; 82 participants; one study; low-certainty evidence; Analysis 3.1).

\subsection{Major bleeding}

Kondratyeva 2010 reported on major bleeding with five cases in the combined treatment group and one case in the standard dose VKA treatment group, resulting in an RR of $7.42(95 \% \mathrm{Cl} 0.91$ to $60.70 ; 82$ participants; one study; low-certainty evidence; Analysis 3.2).

\subsection{All-cause mortality}

Kondratyeva 2010 reported one death in the standard-dose VKAonly group during a median of 51.6 months follow-up for this group, resulting in an RR of 0.49 (95\% $\mathrm{Cl} 0.02$ to 11.68 ; 82 participants; one study; very low-certainty evidence; Analysis 3.3).

\subsection{Stroke}

Kondratyeva 2010 reported three participants with stroke in the combined-treatment group and one participant with stroke in the standard-dose VKA-only group, resulting in an RR of $4.45(95 \% \mathrm{Cl}$ 0.48 to 41.00 ; 82 participants, one study; low-certainty evidence; Analysis 3.4).

\subsection{Transient ischemic attack}

Kondratyeva 2010 reported five participants with TIA in the combined-treatment group and three participants with TIA in the standard-dose VKA-only treatment group, resulting in an RR of 2.47, $95 \% \mathrm{Cl} 0.63$ to 9.66; 82 participants; one study; Analysis 3.5 ).

\subsection{Venous thromboembolism (VTE)}

Kondratyeva 2010 reported one participant with VTE in the combined-treatment group and three participants with VTE in the standard-dose VKA group, resulting in an RR of $0.49(95 \% \mathrm{Cl} 0.05$ to 4.56; 82 participants; one study; Analysis 3.6).

\subsection{Myocardial infarction}

Kondratyeva 2010 reported one participant with myocardial infarction in the combined-treatment group and two participants in the standard-dose VKA-only treatment group, resulting in an RR of 0.74 ( $95 \% \mathrm{Cl} 0.07$ to 7.86 ; 82 participants; one study; Analysis 3.7).

\subsection{Other thrombotic events}

Kondratyeva 2010 reported on three participants with other thrombotic events (hearing loss and retinal artery thrombosis) in the combined-treatment group and no other thrombotic events in the standard-dose VKA-only treatment group, resulting in an RR of 10.29 (95\% Cl 0.55 to 192.97; 82 participants; 1 study; Analysis 3.8).

\subsection{Any bleeding that does not meet the criteria for major bleeding}

Kondratyeva 2010 reported on 20 participants with minor bleeding in the combined-treatment group and 23 in the VKA-only group, resulting in an RR of 1.29 (95\% Cl 0.86 to $1.94 ; 82$ participants; one study; low-certainty evidence; Analysis 3.9).

\section{Standard dose VKA plus single antiplatelet agent versus single antiplatelet agent}

The studies included in this comparison did not report on any thromboembolic events (only on stroke), all-cause death, TIA, VTE, $\mathrm{MI}$, other thrombotic events, quality of life, adverse events other than bleeding.

\subsection{Major bleeding}

Yamazaki 2009 did not provide any information regarding occurrence of major bleeding in participants included in the study, while Okuma 2010 reported a single case of minor cerebral hemorrhage (which we defined as major bleeding) in the single antiplatelet group at a mean of 3.9 years (SD 2.0) of follow-up, which resulted in $\mathrm{RR}$ of 0.40 (95\% $\mathrm{Cl} 0.02$ to $8.78 ; 20$ participants; one study; very low-certainty evidence; Analysis 4.1).

\subsection{Stroke}

Although two studies comparing standard dose VKA plus single antiplatelet agents versus single antiplatelet drug (aspirin) reported stroke as an outcome (Okuma 2010; Yamazaki 2009), only one small study (reported only in conference abstracts) provided results that could be shown on a forest plot (Yamazaki 2009). It did not show significant differences between the treatment groups at one-year follow-up, but the aspirin group was discontinued for "humanitarian" reasons, as all three events took place in this group, while no events occurred in the combined treatment group (RR $0.14,95 \% \mathrm{Cl} 0.01$ to $2.60 ; 40$ participants; one study; very lowcertainty evidence; Analysis 4.2). Okuma 2010 reported significant differences in the cumulative incidence of stroke in 3.9 years mean follow-up (SD 2.0) in favor of the combination group (log-rank test, $P=0.026$ ) but did not report the numbers of participants with an event or HR.

\subsection{Any bleeding that does not meet the criteria for major bleeding}

Yamazaki 2009 did not provide any information regarding the occurrence of any bleeding in participants included in the study, while Okuma 2010 reported a single case of subcutaneous hemorrhage (which we defined as minor bleeding) in the combined treatment group resulting in an RR of $3.60(95 \% \mathrm{Cl} 0.16$ to 79.01; 20 participants; one study; Analysis 4.3), and no cases of gastrointestinal bleeding (no definition or classification provided; very low-certainty evidence) (Analysis 4.4).

\section{Standard dose VKA plus single antiplatelet agent versus dual antiplatelet agent}

The study included in this comparison did not report on any thromboembolic events (only on stroke), major bleeding, all-cause death, TIA, VTE, MI, other thrombotic events, quality of life, any bleeding that does not meet the criteria for major bleeding, or adverse events other than bleeding.

\subsection{Stroke}

Only Yamazaki 2009 (reported in conference abstracts) provided results at three-year follow-up, but there were only two events in the VKA plus antiplatelet group and no events in the dual 
antiplatelet group, resulting in an RR of $5.0(95 \% \mathrm{Cl} 0.26$ to $98.00 ; 40$ participants; one study; very low-certainty evidence; Analysis 5.1).

\section{Dual antiplatelet agent versus single antiplatelet agent}

The study included in this comparison did not report on any thromboembolic events (only on stroke), major bleeding, all-cause death, TIA, VTE, MI, other thrombotic events, quality of life, any bleeding that does not meet the criteria for major bleeding, or adverse events other than bleeding.

\subsection{Stroke}

Only Yamazaki 2009 (reported in conference abstracts) provided results at one-year follow-up. However, the aspirin group was discontinued for "humanitarian" reasons, as all three events occurred in this group, while there were no events in the combined treatment group (RR 0.14, 95\% $\mathrm{Cl} 0.01$ to 2.60; 40 participants; one study; very low-certainty evidence; Analysis 6.1).

\section{DISCUSSION}

\section{Summary of main results}

Of six comparisons reported in this review, three showed some evidence of difference between treatments for several outcomes.

We identified three studies comparing NOAC (rivaroxaban) with standard-dose VKA, which did not show differences between those groups for any thromboembolic event, major and clinically relevant non-major bleeding, and death (moderate-certainty evidence). We found increased risk of stroke with NOAC compared with standard dose VKA (moderate-certainty evidence).

We identified two studies comparing high-dose VKA with standarddose VKA in the secondary prevention of recurrent thrombosis in people with APS. We found that the differences in the rates of thrombotic events or major bleeding between the treatment groups were not statistically significant, but there was some evidence of an increased risk of minor and any bleeding in the highdose group (low-certainty evidence). However, one of those studies was underpowered, and in the other one the rate of thrombosis was lower than expected.

We identified only one small study, at high risk of bias because of lack of allocation concealment, comparing standard-dose VKA plus single antiplatelet agents versus standard-dose VKA. It showed increased risk of any thromboembolic event with combined treatment (low-certainty evidence), and no differences for other outcomes (very low- to low-certainty evidence).

For three comparisons (standard-dose VKA plus single antiplatelet agent versus single antiplatelet agent and versus dual antiplatelet agent and dual antiplatelet agent versus single antiplatelet agent) small, poorly-reported studies at high risk of bias did not provide any conclusive evidence regarding benefits or harms of those drugs in the secondary prevention of stroke in people with APS.

\section{Overall completeness and applicability of evidence}

The studies included in the review were small with very small numbers of events. For the comparison of NOAC with standarddose VKA, the number of events for primary outcomes (thrombotic events and major bleeding) was low and one of the included studies did not report any events. The number of thrombotic events and major bleedings reported for high-dose VKA compared with standard-dose VKA was also low, as was the number of events in a single study comparing standard-dose VKA and antiplatelet therapy with standard-dose VKA. The evidence for antiplatelet agents or a combination of antiplatelet and anticoagulant agents was even poorer, due to very small studies or very limited reporting, or both.

The completeness of data, therefore, is a concern in this review with regard to the effects of antiplatelets or anticoagulants, or both, as several studies did not report the data required for meta-analysis in the assessment of either benefit or harm, and one study was terminated without results published. In addition, we have not been able to finally assess two additional studies due to unclear reporting of methods or data.

All eight included studies reported including people with APS, but qualifying clinical events differed between the studies. Five studies included people with both previous arterial and venous events, two studies included only people with previous stroke, while one study comparing NOAC to the standard-dose VKA included only people with previous venous thromboembolism while taking no or sub-therapeutic doses of anticoagulation treatment and without VTE while on warfarin at INR 2.0 to 3.0 , so its results may not be applicable to people with a previous arterial event related to APS and with the recurrent event despite standard anticoagulation. Although five studies (two comparing high-dose VKA with standarddose VKA, two comparing NOAC with standard-dose VKA and one comparing standard-dose VKA and antiplatelet with standard-dose VKA) included people with both arterial and venous thrombosis, most participants had prior venous thrombosis (64\% to $75 \%$ ). Therefore, those results may not be fully applicable to people with previous arterial thrombosis.

Similarly, two studies comparing the use of anticoagulant plus antiplatelet agents versus a single antiplatelet agent or dual antiplatelet therapy included only people with previous stroke; results may therefore not be applicable to people with previous venous thromboembolism.

The proportions of participants with each type of antibody, and participants positive for two or three types of antibodies, differed among studies. In addition, one study included only people who were positive for three types of antibodies (high-risk patients). This would seem to increase the generalizability of evidence in this review, but due to the small number of studies we could not explore the influence of those factors on the effects of our review's eligible interventions. Therefore, properly designed RCTs, including homogenous thrombotic APS patient populations, are needed. People with various constellations of antibodies may have different outcomes and different responses to treatment.

Additional ongoing studies of NOACs compared with standard anticoagulants, in particular the ASTRO-APS trial (ASTRO-APS), may add to the body of evidence and help to provide higher-certainty evidence on the benefits and harms of using NOACs in secondary prevention of thrombotic events in people with thrombotic APS.

\section{Quality of the evidence}

We analyzed data from eight trials involving 811 participants with APS. All eight trials took place over the previous 20 years. We judged only one study to be at low risk of bias in all domains (Crowther 2003), while another was at low risk of bias in all domains for one 
group of outcomes (RAPS 2016). We judged the other six studies to be at low, high, or unclear risk of selection bias; at low, high, or unclear risk for performance bias; at low, high, or unclear risk for detection bias; low or unclear for attrition bias, and/or reporting bias; or at low, high, or unclear risk of other bias. We did not detect important heterogeneity among the results of the studies. However, the studies differed in duration of follow-up. For the NOAC versus standard-dose VKA comparison, the follow-up ranged between 210 days (RAPS 2016), to 35.4 months (Ordi-Ros 2019), while for high-dose VKA versus standard-dose VKA it ranged from 2.7 years (Crowther 2003), to 3.6 years (WAPS 2005). From a clinical perspective, such differences are acceptable as recurrent events usually occur early in the observation. The RAPS 2016 study differed from the other two studies in the NOAC versus standard-dose VKA comparison in terms of the follow-up, but also due to a surrogate end-point and no clinical events during the follow-up.

All of the analyses provided imprecise results with wide confidence intervals. We could not assess publication bias due to the small number of studies.

We judged the certainty of evidence to be low to moderate for the outcomes in the comparison of NOACs versus standard-dose VKA; low for the outcomes in the comparison of high-dose VKA and standard-dose VKA; low to very low for the comparison of standard-dose VKA plus antiplatelets with standard-dose VKA. For all outcomes in other comparisons, we considered the evidence certainty to be very low. We downgraded the evidence mostly for risk of bias, imprecision, or both.

\section{Potential biases in the review process}

In our comprehensive searches, supplemented by seeking additional information from experts, unpublished sources, and manufacturers, we attempted to identify all RCTs of potential relevance to the review. We did not apply any limitations to our searches, and for studies published in a language in which none of the review authors was fluent, we sought help with translation. In fact, when we compared the number of studies identified in other recent reviews, meta-analyses, or practice guidelines, our review identified more studies (published, ongoing, awaiting classification) than previous published reviews (Alegria 2010; Da Silva 2015; Danowski 2013; Dufrost 2016; Erkan 2014; Keeling 2012; Kim 2016; Ruiz-Irastorza 2011).

Due to the very small number of included studies in each comparison, we did not produce funnel plots.

\section{Agreements and disagreements with other studies or reviews}

We identified several recent reviews that covered topics similar to our review. Da Silva 2015 compared high- and moderate-intensity warfarin treatment on the basis of two studies that were also included in our review (Crowther 2003; WAPS 2005), concluding that moderate-intensity anticoagulation is more suitable for people with APS. However, they based their conclusion on the findings of higher rates of thrombotic events in the high-intensity group, which was probably an error, as the number of events on the forest plot does not match the number of events reported by the study; and on a higher risk of minor bleeding on the basis of pooled results for both studies. In fact Crowther 2003 did not report minor bleeding events.
Dufrost 2016 examined effects of NOACs in APS and, in addition to RAPS 2016 (which we also identified), this review included case reports and case series. They concluded that NOACs should be used with caution in people with APS and called for additional RCTs with clinical primary endpoints. Authors of patient-level data meta-analysis based on all types of studies (including case series, case-reports, and post-hoc subgroup analyses of RCTs) observed more thrombotic events in APS patients taking NOACs, especially in those with more criteria for definite APS, with previous arterial thrombosis, and with triple antibody positivity. This might suggest that a high-risk aPL antibody profile is associated with lower effectiveness of NOACs (Dufrost 2018). We should, however, await the result of still-ongoing RCTs comparing NOACs to standard care in APS to be able to draw conclusions applying to the whole group of these drugs.

Kim 2016 focused on the intensity of warfarin anticoagulation in people with APS and included several retrospective studies and two RCTs that we also included. That review concluded that more evidence is required with larger sample sizes and better adherence to treatment. We agree that weak adherence to treatment, expressed as INR outside the therapeutic range, is a significant flaw of studies evaluating effects of VKA treatment in APS.

Tektonidou 2019b published systematic reviews which supported EULAR recommendations on the management of adults with APS. They reviewed evidence up to January 2018 on both primary and secondary prevention and included a total of 188 studies of various designs. For comparisons of high-dose VKA versus standard-dose VKA, they included RCTs also identified in our review. while for the analysis of the effect of NOAC they included both RCTs published up to 2018 , which we also identified. They concluded that there is a need for properly designed studies with homogenous APS populations including patients with single- or double-positive APS.

\section{AUTHORS' CONCLUSIONS}

\section{Implications for practice}

The evidence identified in this systematic review indicates that NOAC compared with standard-dose VKA probably increases risk of stroke and likely does not alter the risk of other adverse outcomes, such as any thromboembolic events, major bleeding, mortality, clinically-relevant non-major bleeding (moderatecertainty evidence). The evidence suggests that NOAC compared with standard-dose VKA may result in little to no difference in quality of life (low-certainty evidence).

The evidence suggests that using high-dose versus standard-dose VKA may not alter the risk of any thromboembolic events, major bleeding, death, and stroke and may increase the risk of any bleeding (low-certainty evidence).

The evidence suggests that standard-dose VKA combined with antiplatelet agent compared with standard-dose VKA only may increase the risk of any thromboembolic event and may not alter the risk of major bleeding, stroke, and minor bleeding (lowcertainty evidence). The evidence is very uncertain about the effect of combined VKA and antiplatelet on the risk of death (very lowcertainty evidence). 
The evidence is very uncertain about the benefit or harm of using standard-dose VKA plus antiplatelet versus single or dual antiplatelet therapy, or dual versus single antiplatelet therapy, for the secondary prevention of recurrent thrombosis in people with APS (very low-certainty evidence).

\section{Implications for research}

Future research should be adequately powered and ensure proper adherence to treatment to assess the effects of the intervention on clinically-important outcomes in people with APS, enabling meaningful conclusions regarding the effects of antiplatelet and anticoagulant agents and their intensity. The thrombotic risk in APS depends on many factors, including the type and number of positive aPL antibodies. Therefore, there is a need for studies with adequate patient stratification as the response to treatment might be different in people with high- and low-risk antibody profile or people with previous arterial or venous thrombotic events. There is a special need to evaluate the efficacy and safety of other NOAC agents (i.e. dabigatran, apixaban, edoxaban) versus standard care for treating APS.

\section{ACK N O WLEDGEMENTS}

We thank Magdalena Koperny and Joshua Cheyne for their review and contributions to the search strategy and acquisition of fulltext articles, Dr Karsten Juhl Jørgensen for comments on the draft protocol, Anna Witkowska for help with managing the references and obtaining full-text articles, staff of the Medical Library of Jagiellonian University Medical College for help with obtaining fulltext articles, trainee reviewers who helped with title and abstract screening or full-text screening: Dawid Storman, Monika Storman, Anastazja Szlauer, and Joanna Zajac. We would like to thank Prof Liliya Eugenevna Ziganshina for help with the data extraction and risk-of-bias assessment for the study published in Russian and Prof Tatiana Reshetnyak for providing additional information about the study.

We thank Prof Guido Finazzi for help with resolving issues concerning the WAPS 2005 study, Dr Josefina Cortés Hernández for help regarding the Ordi-Ros 2019 study, Prof Pier L Meroni, Prof Munther A Khamashta, and Prof Phillippe de Moerloose, Prof Vittorio Pengo, and representatives of Bayer and Bristol-MeyersSquibb/Pfizer for help with checking for additional studies. 


\section{R E F E R E N C E S}

\section{References to studies included in this review}

\section{Crowther 2003 \{published data only\}}

Anonymous. Erratum. A comparison of two intensities of warfarin for the prevention of recurrent thrombosis in patients with the antiphospholipid antibody syndrome. New England Journal of Medicine 2003;349(26):2577.

Crowther MA, Ginsberg JS, Gent M, Julian J, Costantini L, Kovacs $\mathrm{M}$, et al. A randomized trial of two intensities of warfarin (international normalized ratio of 2.0 to 3.0 versus 3.1 to 4.0 ) for the prevention of recurrent thrombosis in patients with antiphospholipid antibodies. Blood 2002;100(2):148a.

* Crowther MA, Ginsberg JS, Julian J, Denburg J, Hirsh J, Douketis J, et al. A comparison of two intensities of warfarin for the prevention of recurrent thrombosis in patients with the antiphospholipid antibody syndrome. New England Journal of Medicine 2003;349(12):1133-8.

\section{Kondratyeva 2010 \{published and unpublished data\}}

Kondratyeva L, Reshetnyak T. The use of warfarin and low-dose aspirin in patients with antiphospholipid syndrome. Lupus 2010;19(4):544-5.

* Kondratyeva LV, Patrusheva NL, Patrushev L, Aleksandrova EN, Kovalenkro TF, Ostryakova EV, et al. Recurrences of thromboses and hemorrhagic complications in patients with antiphospholipid syndrome during therapy with warfarin plus aspirin [РЕЦИДИВЫ ТРОМБОЗОВ И ГЕМОРРАГИЧЕСКИХ ОСЛОЖНЕНИЙ У БОЛЪНЫХ С АНТИФОСФОЛИПИДНЫМ СИНДРОМОМ НА ФОНЕ ТЕРАПИИ ВАРФАРИНОМ И АСПИРИНОМ]. Terapevticheskii Arkhiv 2010;82(5):33-9.

Reshetnyak TM, Kondratyeva LV, Patrusheva NL, Patrushev LI. Warfarin in the treatment of antiphospholipid syndrome [ВАРФАРИН ПРИ ЛЕЧЕНИИ АНТИФОСФОЛИПИДНОГО СИНДРОМА]. Terapevticheskii Arkhiv 2007;79(5):47-54.

\section{Okuma 2010 \{published data only\}}

* Okuma H, Kitagawa Y, Yasuda T, Tokuoka K, Takagi S. Comparison between single antiplatelet therapy and combination of anti-platelet and anticoagulation therapy for secondary prevention in ischemic stroke patients with antiphospholipid syndrome. International Journal of Medical Sciences 2010;7(1):15-8.

Okuma H, lijima K, Goto Y, Yasuda T, Tokuoka K, Kitagawa Y. Prevention in ischemic stroke with antiphospholipid syndrome. In: Proceedings of the International Stroke Conference 2013. 6-8 February 2013; Honolulu, Hawaii, USA. 2013.

\section{Ordi-Ros 2019 \{published data only\}}

2010-019764-36. Rivaroxaban vs acenocumarol in secondary prophylaxis of antiphospholipid syndrome: multicenter, randomized, prospective study. [Rivaroxaban versus acenocumarol en la profilaxis secundaria del síndrome antifosfolípido: un estudio multicéntrico, prospectivo y randomizado]. www.clinicaltrialsregister.eu/ctr-search/ trial/2010-019764-36/ES (first received 2 November 2010).
Cortes-Hernandez J, Saez-Comet L, Mestre AR, Salomo AC, Pedragosa JC, Ortiz-Santamaria V, et al. Rivaroxaban versus warfarin as secondary thromboprophylaxis in patients with antiphospholipid syndrome: a randomized, multicenter, openlabel, clinical trial. Arthritis and Rheumatology 2018;70 Suppl 10:183.

Cortes-Hernandez J, Saez-Comet L, Perez-Conesa M, Riera Mestre A, Castro-Salomo A, Parra S, et al. Rivaroxaban versus warfarin as secondary thromboprophylaxis in patients with antiphospholipid syndrome protocol: a randomized, multicentre, open-label, clinical trial. Annals of the Rheumatic Diseases 2017;76 Suppl 2:1208.

NCT02926170. Rivaroxaban versus acenocumarol for secondary thromboprophylaxis in patients with antiphospholipid syndrome: a randomized, prospective, phase III study. Analysis of stratification prognostic factors. clinicaltrials.gov/show/ NCT02926170 (first received: 3 October 2016).

* Ordi-Ros J, Sáez-Comet L, Pérez-Conesa M, Vidal X, Riera-Mestre A, Castro-Salomó A, et al. Rivaroxaban versus vitamin $\mathrm{K}$ antagonist in antiphospholipid syndrome: a randomized noninferiority trial. Annals of Internal Medicine 2019;171(10):685-94.

\section{RAPS 2016 \{published and unpublished data\}}

2012-002345-38. A prospective randomised controlled phase II/ III clinical trial of rivaroxaban versus warfarin in patients with thrombotic antiphospholipid syndrome, with or without SLE. www.clinicaltrialsregister.eu/ctr-search/trial/2012-002345-38/ GB/ (first entered in the EudraCT database: 08 October 2012).

Arachchillage DJ, Mackie I, Efthymiou M, Chitolie A, Hunt B, Isenberg D, et al. Rivaroxaban limits complement activation in patients with thrombotic antiphospholipid syndrome. Journal of Thrombosis and Haemostasis 2015;13(S2):281.

Arachchillage DJ, Mackie IJ, Efthymiou M, Chitolie A, Hunt BJ, Isenberg D, et al. Rivaroxaban limits complement activation compared to warfarin in antiphospholipid syndrome patients with venous thromboembolism. Blood 2015;126(23):2328.

Arachchillage DJ, Mackie IJ, Efthymiou M, Chitolie A, Hunt BJ, et al. Rivaroxaban limits complement activation compared with warfarin in antiphospholipid syndrome patients with venous thromboembolism. Journal of Thrombosis and Haemostasis 2016;14(11):2177-86.

Cohen H, Dore C, Clawson S, Hunt BJ, Khamashta M, Machin SJ, et al. A prospective randomised controlled phase II/III clinical trial of rivaroxaban vs. warfarin in patients with thrombotic antiphospholipid syndrome, with or without SLE. Journal of Thrombosis and Haemostasis 2013;11(s2):860.

Cohen H, Dore CJ, Clawson S, Hunt BJ, Isenberg D, Khamashta M, et al. Rivaroxaban in antiphospholipid syndrome (RAPS) protocol: a prospective, randomized controlled phase II/III clinical trial of rivaroxaban versus warfarin in patients with thrombotic antiphospholipid syndrome, with or without SLE. Lupus 2015;24(10):1087-94. 
Cohen H, Hunt B, Efythymiou M, Arachchillage D, Ruiz M, Clawson S, et al. A prospective randomised controlled phase II/III clinical trial of rivaroxaban vs. warfarin in patients with thrombotic antiphospholipid syndrome, with or without SLE. Journal of Thrombosis and Haemostasis 2015;13(S2):84.

* Cohen H, Hunt B, Efythymiou M, Arachchillage D, Ruiz M, Clawson S, et al. Rivaroxaban versus warfarin to treat patients with thrombotic antiphospholipid syndrome, with or without systemic lupus erythematosus (RAPS): a randomised, controlled, open-label, phase $2 / 3$, non-inferiority trial. Lancet Haematology 2016;3(9):e426-36.

ISRCTN68222801. A prospective randomised controlled phase II/III clinical trial of rivaroxaban versus warfarin in patients with thrombotic antiphospholipid syndrome, with or without systemic lupus erythematosus (RAPS). https://www.isrctn.com/ ISRCTN68222801 (date applied: 17/10/2012).

\section{TRAPS 2016 \{published data only\}}

2013-004575-13. A prospective, randomized clinical trial comparing rivaroxaban vs warfarin in high risk patients with Antiphospholipid Syndrome (TRAPS) [Studio clinico prospettico, randomizzato di confronto tra Rivaroxaban vs Warfarin in pazienti ad alto rischio con sindrome da anticorpi antifosfolipidi (TRAPS)]. www.clinicaltrialsregister.eu/ctrsearch/trial/2013-004575-13/IT (first entered in the EudraCT database: 19 September 2014)

NCT02157272. A prospective, randomized clinical trial comparing rivaroxaban vs warfarin in high risk patients with antiphospholipid syndrome. clinicaltrials.gov/show/ NCT02157272 (first received: 31 May 2014).

* Pengo V, Banzato A, Bison E, Zoppellaro G, Padayattil Jose S, Denas $G$. Efficacy and safety of rivaroxaban vs warfarin in high-risk patients with antiphospholipid syndrome: rationale and design of the Trial on Rivaroxaban in AntiPhospholipid Syndrome (TRAPS) trial. Lupus 2016;25(3):301-6.

Pengo V, Denas G, Zoppellaro G, Jose SP, Hoxha A, Ruffatti A, et al. Rivaroxaban vs warfarin in high-risk patients with antiphospholipid syndrome. Blood 2018;132(13):1365-71.

\section{WAPS 2005 \{published and unpublished data\}}

Finazzi G, Barbui T. Feasibility of a randomized clinical trial for the prevention of recurrent thrombosis in the antiphospholipid syndrome. Annales de Medecine Interne 1996;147(Suppl 1):38-41.

Finazzi G, Barbui T. Proposal of an international clinical trial of high-dose warfarin in the antiphospholipid syndrome (APS). British Journal of Haematology 1996;93(S2):315.

* Finazzi G, Brancaccio V, Schinco P, Wisloff F, Musial J, Baudo F, et al. A randomized clinical trial of high-intensity warfarin vs. conventional antithrombotic therapy for the prevention of recurrent thrombosis in patients with the antiphospholipid syndrome (WAPS). Journal of Thrombosis and Haemostasis 2005;3(5):848-53.

Finazzi G, Marchioli R, Barbui T. A randomized clinical trial of oral anticoagulant therapy in patients with the antiphospholipid syndrome: the WAPS study [abstract]. Journal of Thrombosis and Haemostasis 2003;1(Suppl 1):OC365.

Finazzi G, Marchioli R, Barbui T. A randomized clinical trial of two intensities of oral anticoagulant therapy in patients with the antiphospholipid syndrome: final results of the WAPS. Blood 2003;102(11 Part 1):16a.

Finazzi G, Marchioli R, Barbui T. The WAPS trial (warfarin in the antiphospholipid syndrome): study population and preliminary results. Hematology Journal 2002;1 Suppl 1:350.

Finazzi G, Marchioli R. The WAPS (warfarin in antiphospholipid syndrome) study. Update and preliminary results. Hematology Journal 2000;1 Suppl 1:135.

Triplett D (as supplied by Cochrane Stroke Group 17 January 2017). Warfarin for the prevention of recurrent thrombosis in the AntiPhospholipid Syndrome. Data on file.

\section{Yamazaki 2009 \{published data only\}}

* Yamazaki M, Kadohira Y, Maekawa M, Hayashi T, Morishita E, Asakura $\mathrm{H}$, et al. Combined antiplatelet agents might help prevent arterial thromboses in antiphospholipid syndrome. Journal of Thrombosis and Haemostasis 2009;7m Suppl 2:720-1.

Yamazaki M, Kadohira Y, Maekawa M, Hayashi T, Morishita E, Asakura $\mathrm{H}$, et al. Plasma levels of platelet-derived microparticles (PDMP) might comprise a useful indicator for antiplatelet therapy in antiphospholipid syndrome (APS). Thrombosis and Haemostasis 2009;7 Suppl 2:951.

Yamazaki M. Plasma levels of platelet-derived microparticle (PDMP) may be an useful indicator of antiplatelet therapy in antiphospholipid syndrome (APS). Lupus 2010;19(4):541.

\section{References to studies excluded from this review}

Cuadrado 2009 \{published data only\}

* Cuadrado MJ, Bertolaccini ML, Seed P, Tektonidou M, Aguirre A, Mico L, et al. Primary prevention of thrombosis in antiphospholipid antibodies positive patients: a prospective, multicenter, randomised, open trial comparing low dose aspirin with low dose aspirin plus low intensity oral anticoagulation. Arthritis \& Rheumatology 2009;60(s10):1285.

Cuadrado MJ, Bertolaccini ML, Seed PT, Tektonidou MG, Aguirre A, Mico L, et al. Low-dose aspirin vs low-dose aspirin plus low-intensity warfarin in thromboprophylaxis: a prospective, multicentre, randomized, open, controlled trial in patients positive for antiphospholipid antibodies (ALIWAPAS). Rheumatology 2014;53(2):275-84.

\section{References to studies awaiting assessment}

JASPRES \{published data only\}

C000000342. Japan Antiphospholipid syndrome-Stroke Prevention Study. upload.umin.ac.jp/cgi-open-bin/ctr_e/ ctr_view.cgi?recptno=R000000395 (first received: 01 March 2006). 
Okuma 2014 \{published data only\}

Okuma H, Kodera Y, Yasuda T, Tokuoka K, Kitagawa Y. Preventive stroke strategies antiphospholipid syndrome in ischemic stroke patients. International Journal of Stroke 2014;9(S3):282.

\section{Yamazaki 2007 \{published data only\}}

Yamazaki M, Kadohira Y, Morishita E, Asakura H, Nakao S. Combined antithrombotic therapy but not single-agent therapy with low-dose aspirin deteriorated platelet activation in patients with antiphospholipid syndrome (APS). Blood 2007;110(11):4002.

\section{References to ongoing studies}

\section{ASTRO-APS \{published data only\}}

NCT02295475. Apixaban for the secondary prevention of thromboembolism: a prospective randomized outcome pilot study among patients with the AntiphosPholipid Syndrome. clinicaltrials.gov/show/NCT02295475 (first received: 18 November 2014).

* Woller SC, Stevens SM, Kaplan DA, Branch DW, Aston VT, Wilson EL, et al. Apixaban for the secondary prevention of thrombosis among patients with Antiphospholipid Syndrome: study rationale and design (ASTRO-APS). Clinical and Applied Thrombosis/Hemostasis 2016;22(3):239-47.

Woller SC, Stevens SM, Kaplan DA, Rondina MT. Protocol modification of apixaban for the secondary prevention of thrombosis among patients with antiphospholipid syndrome study. Clinical and Applied Thrombosis/Hemostasis 2018;24:192.

\section{RISAPS \{published data only\}}

ISRCTN10280992. Rivaroxaban for stroke patients with antiphospholipid syndrome. isrctn.com/ISRCTN10280992 (date assigned: 25 April 2019).

* NCT03684564. Rlvaroxaban for Stroke Patients With AntiPhospholipid Syndrome (RISAPS). clinicaltrials.gov/ct2/ show/nct03684564 (first posted: 25 September 2018).

\section{Additional references}

\section{Advisory Council 1975}

Advisory Council for the National Institute of Neurological and Communicative Disorders and Stroke II . Classification and outline of cerebrovascular diseases II. Stroke 1975;6(5):564-616.

\section{Ageno 2012}

Ageno W, Gallus AS, Wittkowsky A, Crowther M, Hylek EM, Palareti G, American College of Chest Physicians. Oral anticoagulant therapy, antithrombotic therapy and prevention of thrombosis, 9th edition. American College of Chest Physicians Evidence-Based Clinical Practice Guidelines. Chest 2012;141(2 Suppl):e44S-e88S.

\section{Alegria 2010}

Alegria MA, Arauz A, Roa LF, Marquez J, Calleja J, Leyva A, et al. Antiocoagulation for the secondary prevention of ischemic stroke [Anticoagulación en la prevención secundaria de la enfermedad vascular cerebral]. Revista de Investigación Clínica 2010;62(2):141-51.

\section{Andreoli 2013}

Andreoli L, Chighizola CB, Banzato A, Pons-Estel GJ, Ramire de Jesus G, Erkan DSO. Estimated frequency of antiphospholipid antibodies in patients with pregnancy morbidity, stroke, myocardial infarction, and deep vein thrombosis: a critical review of the literature. Arthritis Care \& Research 2013;65(11):1869-73.

\section{APASS Investigators 2004}

APASS Investigators. Antiphospholipid antibodies and subsequent thrombo-occlusive events in patients with ischemic stroke. JAMA 2004;291(5):576-84.

\section{Bala 2018}

Bala MM, Paszek EM, Lesniak W, Wloch-Kopec D, Jasinska K, Undas A. Antiplatelet and anticoagulant agents for primary prevention of thrombosis in individuals with antiphospholipid antibodies. Cochrane Database of Systematic Reviews 2018, Issue 7. Art. No: CD012534. [DOI: 10.1002/14651858.CD012534]

\section{Bushnell 2000}

Bushnell CD, Goldstein LB. Diagnostic testing for coagulopathies in patients with ischemic stroke. Stroke 2000;31(12):3067-78.

\section{Cervera 2002}

Cervera R, Piette JC, Font J, Khamashta MA, Shoenfeld Y, Camps MT, et al. Antiphospholipid syndrome: clinical and immunologic manifestations and patterns of disease expression in a cohort of 1,000 patients. Arthritis and Rheumatism 2002;46(4):1019-27.

\section{Cervera 2009}

Cervera R, Khamashta MA, Shoenfeld Y, Camps MT, Jacobsen S, Kiss $\mathrm{E}$, et al. Morbidity and mortality in the antiphospholipid syndrome during a 5-year period: a multicentre prospective study of 1000 patients. Annals of the Rheumatic Diseases 2009;68(9):1428-32.

\section{Cervera 2010}

Cervera R. Update on the diagnosis, treatment, and prognosis of the catastrophic antiphospholipid syndrome. Current Rheumatology Reports 2010;12(1):70-6.

\section{Chatzikonstantinou 2013}

Chatzikonstantinou A, Wolf ME, Schaefer A, Hennerici MG. Risk prediction of subsequent early stroke in patients with transient ischemic attacks. Cerebrovascular Diseases 2013;36(2):106-9.

\section{Chighizola 2014}

Chighizola CB, Moia M, Meroni PL. New oral anticoagulants in thrombotic antiphospholipid syndrome. Lupus 2014;23(12):1279-82.

\section{Chighizola 2015}

Chighizola CB, Ubiali T, Meroni PL. Treatment of thrombotic antiphospholipid syndrome: the rationale of current management - an insight into future approaches. Journal 
of Immunology Research 2015;2015:ID: 951424. [DOI:

10.1155/2015/951424]

\section{Covidence [Computer program]}

Veritas Health Innovation Covidence. Melbourne, Australia: Veritas Health Innovation, (accessed 25 March 2017). Available at www.covidence.org.

\section{Da Silva 2015}

Da Silva FF, de Carvalho JF. Intensity of anticoagulation in the treatment of thrombosis in the antiphospholipid syndrome: a meta-analysis. Revista Brasileira de Reumatologia (English Edition) 2015;55(2):159-66.

\section{Danowski 2013}

Danowski A, Rego J, Kakehasi AM, Funke A, Carvalho JF, Lima IV, et al. Guidelines for the treatment of antiphospholipid syndrome. Revista Brasileira de Reumatologia (English Edition) 2013;53(2):184-92.

\section{Deeks 2001}

Deeks JJ, Altman DG, Bradburn MJ. Statistical methods for examining heterogeneity and combining results from several studies in meta-analysis. In: Egger M, Davey Smith G, Altman DG editor(s). Systematic Reviews in Health Care. 2nd Edition.. London, UK: BMJ Books, 2001:200.

\section{Der Simonian 1986}

DerSimonian R, Laird N. Meta-analysis in clinical trials. Controlled Clinical Trials 1986;7(3):177-88.

\section{Dufrost 2016}

Dufrost V, Risse J, Zuily S, Wahl D. Direct oral anticoagulants use in antiphospholipid syndrome: are these drugs an effective and safe alternative to warfarin? A systematic review of the literature. Current Rheumatology Reports 2016;18(12):74.

\section{Dufrost 2018}

Dufrost V, Risse J, Reshetnyak T, Satybaldyeva M, Du Y, Yan XX, et al. Increased risk of thrombosis in antiphospholipid syndrome patients treated with direct oral anticoagulants. Results from an international patient-level data meta-analysis. Autoimmunity Reviews 2018;17(10):1011-21.

\section{Easton 2009}

Easton JD, Saver JL, Albers GW, Alberts MJ, Chaturvedi S, Feldmann E, American Heart Association, American Stroke Association Stroke Council, Council on Cardiovascular Surgery and Anesthesia, Council on Cardiovascular Radiology and Intervention, Council on Cardiovascular Nursing, Interdisciplinary Council on Peripheral Vascular Disease. Definition and evaluation of transient ischemic attack: a scientific statement for healthcare professionals from the American Heart Association/American Stroke Association Stroke Council; Council on Cardiovascular Surgery and Anesthesia; Council on Cardiovascular Radiology and Intervention; Council on Cardiovascular Nursing; and the Interdisciplinary Council on Peripheral Vascular Disease. Stroke 2009;40(6):2276-93.

\section{Erkan 2007}

Erkan D, Harrison MJ, Levy R, Peterson M, Petri M, Sammaritano $\mathrm{L}$, et al. A randomized double blind placebo controlled trial in asymptomatic antiphospholipid antibody patients. Arthritis \& Rheumatism 2007;56(7):2382-91.

\section{Erkan 2014}

Erkan D, Aguiar CL, Andrade D, Cohen H, Cuadrado MJ, Danowskie A, et al. 14th International Congress on Antiphospholipid Antibodies: task force report on antiphospholipid syndrome treatment trends. Autoimmunity Reviews 2014;13(6):685-96.

\section{Espinosa 2015}

Espinosa G, Cervera R. Current treatment of antiphospholipid syndrome: lights and shadows. Nature Reviews Rheumatology 2015;11(10):586-96.

\section{Finazzi 2005}

Finazzi G, Marchioli R, Brancaccio V, Schinco P, Wisloff F, Musial J, et al. A randomized clinical trial of high-intensity warfarin vs conventional antithrombotic therapy for the prevention of recurrent thrombosis in patients with the antiphospholipid syndrome (WAPS). Journal of Thrombosis and Haemostasis 2005;3(5):848-53.

\section{Galli 2003}

Galli M, Luciani D, Bertolini GL. Lupus anticoagulants are stronger risk factors for thrombosis than anticardiolipin antibodies in the antiphospholipid syndrome: a systematic review of the literature. Blood 2003;101(5):1827-32.

\section{George 2009}

George D, Erkan D. Antiphospholipid syndrome. Progress in Cardiovascular Diseases 2009;52(2):115-25.

\section{Giannakopoulos 2007}

Giannakopoulos B, Passam F, Rahgozar S, Krilis SA. Current concepts on the pathogenesis of the antiphospholipid syndrome. Blood 2007;109(2):422-30.

\section{Giannakopoulos 2013}

Giannakopoulos B, Krilis S. The pathogenesis of the antiphospholipid syndrome. New England Journal of Medicine 2013;368(11):1033-44.

\section{Gomez-Puerta 2014}

Gómez-Puerta JA, Cervera R. Diagnosis and classification of the antiphospholipid syndrome. Journal of Autoimmunity 2014;48-49:20-5.

\section{GRADEpro [Computer program]}

McMaster University GRADEpro GDT: GRADEpro Guideline Development Tool. McMaster University, 2015 (developed by Evidence Prime, Inc.). Available from gradepro.org.

\section{Greenland 1985}

Greenland S, Robins JM. Estimation of a common effect parameter from sparse follow-up data. Biometrics 1985;41(1):55-68. 


\section{Hamulyak 2020}

Hamulyák EN, Scheres LJJ, Marijnen MC, Goddijn M, Middeldorp S. Aspirin or heparin or both for improving pregnancy outcomes in women with persistent antiphospholipid antibodies and recurrent pregnancy loss. Cochrane Database of Systematic Reviews 2020, Issue 5. Art. No: CD012852. [DOI: 10.1002/14651858.CD012852.pub2]

\section{Hasan 2015}

Hasan SS, Teh KM, Ahmed SI, Chong DW, Ong HC, Naina B. Quality of life (QoL) and International Normalized Ratio (INR) control of patients attending anticoagulation clinics. Public Health 2015;129(7):954-62.

\section{Higgins 2011}

Higgins JPT, Green S (editors). Cochrane Handbook for Systematic Reviews of Interventions Version 5.1.0 (updated March 2011). The Cochrane Collaboration, 2011. Available from www.cochrane-handbook.org.

\section{Hirsh 2001}

Hirsh J, Anand SS, Halperin JL, Fuster V. Mechanism of action and pharmacology of unfractionated heparin. Arteriosclerosis, Thrombosis and Vascular Biology 2001;21(7):1094-6.

\section{Islam 2016}

Islam MA, Alam F, Sasongko TH, Husin A, Abdullah S, Gan SH, et al. Antiplatelet and anticoagulant agents for preventing recurrence of peripheral vascular thrombosis in patients with Antiphospholipid syndrome. Cochrane Database of Systematic Reviews 2016, Issue 5. Art. No: CD012198. [DOI: 10.1002/14651858.CD012198]

\section{Iwaniec 2016}

Iwaniec T, Kaczor MP, Celińska-Löwenhoff M, Polański S, Musiat J. Identification of patients with triple antiphospholipid antibody positivity is platform and method independent. Polskie Archiwum Medycyny Wewnetrznej 2016;126(1-2):19-24.

\section{Keeling 2012}

Keeling D, Mackie I, Moore GW, Greer IA, Greaves M, British Committee for Standards in Haematology. Guidelines on the investigation and management of antiphospholipid syndrome. British Journal of Haematology 2012;157(1):47-58.

\section{Kim 2016}

Kim E, Do T, Peacock K, Takundwa PT. Recommended therapeutic INR range for patients with antiphospholipid syndrome on warfarin anticoagulation: is moderateintensity (INR 2.0-3.0) or high-intensity (INR 3.1-4.0) better for reducing risk of recurrent thromboembolic events? Cureus 2016;8(9):e765.

\section{Konstantinides 2020}

Konstantinides SV, Meyer G, Becattini C, Bueno H, Geersing GJ, Harjola VP, ESC Scientific Document Group. 2019 ESC Guidelines for the diagnosis and management of acute pulmonary embolism developed in collaboration with the European Respiratory Society (ERS). European Heart Journal 2020;41(4):543-603.

\section{Lim 2006}

Lim W, Crowther MA, Eikelboom JW. Management of antiphospholipid antibody syndrome - a systematic review. JAMA 2006;295(9):1050-7.

\section{Mantel 1959}

Mantel N, Haenszel W. Statistical aspects of the analysis of data from retrospective studies of disease. Journal of the National Cancer Institute 1959;22(4):719-48.

\section{Matyja-Bednarczyk 2014}

Matyja-Bednarczyk A, Swadzba J, Iwaniec T, Sanak M, Dziedzina S, Cmiel A, et al. Risk factors for arterial thrombosis in antiphospholipid syndrome. Thrombosis Research 2014;133(2):173-6.

\section{Miyakis 2006}

Miyakis S, Lockshin MD, Atsumi T, Branch DW, Brey RL, Cervera R. International consensus statement on an update of the classification criteria for definite antiphospholipid syndrome (APS). Journal of Thrombosis and Haemostasis 2006;4(2):295-306.

\section{Moher 2009}

Moher D, Liberati A, Tetzlaff J, Altman DG, The PRISMA Group. Preferred reporting items for systematic reviews and metaanalyses: the PRISMA statement. BMJ 2009;339:332-6. [DOI: 10.1136/bmj.b2535]

\section{Nalli 2014}

Nalli C, Andreoli L, Casu C, Tincani A. Management of recurrent thrombosis in antiphospholipid syndrome. Current Rheumatology Reports 2014;16(3):405.

\section{Panichpisal 2012}

Panichpisal K, Rozner E, Levine SR. The management of stroke in antiphospholipid syndrome. Current Rheumatology Reports 2012;14(1):99-106.

\section{Parmar 1998}

Parmar MKB, Torri V, Stewart L. Extracting summary statistics to perform meta-analyses of the published literature for survival endpoints. Statistics in Medicine 1998;17(24):2815-34.

\section{Pengo 2010}

Pengo V, Ruffatti A, Legnani C, Resele P, Barcellona D, Erba N, et al. Clinical course of high-risk patients diagnosed with antiphospholipid syndrome. Journal of Thrombosis and Haemostasis 2010;8(2):237-42.

\section{Pengo 2011}

Pengo V, Ruffatti A, Legnani C, Testa S, Fierro T, Marongiu F, et al. Incidence of a first thromboembolic event in asymptomatic carriers of high-risk antiphospholipid antibody profile: a multicenter prospective study. Blood 2011;118(17):4714-8.

\section{Pengo 2015}

Pengo V, Denas G, Padayattil SJ, Zoppellaro G, Bison E, Banzato A, Hoxha A, Ruffatti A. Diagnosis and therapy of antiphospholipid syndrome. Polskie Archiwum Medycyny Wewnetrznej 2015;125(9):672-7. 


\section{Pengo 2018}

Pengo V, Denas G, Zoppellaro G, Jose SP, Hoxha A, Ruffatti A, et al. Rivaroxaban vs warfarin in high-risk patients with antiphospholipid syndrome. Blood 2018;132:1365-71.

\section{Petri 2000}

Petri M. Epidemiology of the antiphospholipid antibody syndrome. Journal of Autoimmunity 2000;15(2):145-51.

\section{Raschi 2016}

Raschi E, Bianchin M, Ageno W, De Ponti R, De Ponti F. Adverse events associated with the use of direct-acting oral anticoagulants in clinical practice: beyond bleeding complications. Polskie Archiwum Medycyny Wewnetrznej 2016;126(7-8):552-61.

\section{RevMan 2014 [Computer program]}

The Nordic Cochrane Centre, The Cochrane Collaboration Review Manager (RevMan). Version 5.3. Copenhagen: The Nordic Cochrane Centre, The Cochrane Collaboration, 2014.

\section{Ruiz-Irastorza 2010}

Ruiz-Irastorza G, Crowther M, Branch W, Khamashta MA. Antiphospholipid syndrome. Lancet 2010;376(9751):1498-509.

\section{Ruiz-Irastorza 2011}

Ruiz-Irastorza G, Cuadrado MJ, Ruiz-Arruza I, Brey R, Crowther M, Derksen R, et al. Evidence-based recommendations for the prevention and long-term management of thrombosis in antiphospholipid antibody-positive patients: report of a task force at the 13th International Congress on antiphospholipid antibodies. Lupus 2011;20(2):206-18.

\section{Saraiva 2015}

Saraiva Sda S, Custódio IF, Mazetto Bde M, Collela MP, de Paula EV, Appenzeller S, et al. Recurrent thrombosis in antiphospholipid syndrome may be associated with cardiovascular risk factors and inflammatory response. Thrombosis Research 2015;136(6):1174-8.

\section{Schulman 2005}

Schulman S, Kearon C, Subcommittee on Control of Anticoagulation of the Scientific and Standardization Committee of the International Society on Thrombosis and Haemostasis. Definition of major bleeding in clinical investigations of antihemostatic medicinal products in nonsurgical patients. Journal of Thrombosis and Haemostasis 2005;3(4):692-4.

\section{Tektonidou 2019a}

Tektonidou MG, Andreoli L, Limper M, Amoura Z, Cervera R, Costedoat-Chalumeau $\mathrm{N}$, et al. EULAR recommendations for the management of antiphospholipid syndrome in adults. Annals of the Rheumatic Diseases 2019;78(10):1296-304.

\section{Tektonidou 2019b}

Tektonidou MG, Andreoli L, Limper M, Tincani A, Ward MM. Management of thrombotic and obstetric antiphospholipid syndrome: a systematic literature review informing the EULAR recommendations for the management of antiphospholipid syndrome in adults. RMD Open 2019;5(1):e000924. doi: 10.1136/ rmdopen-2019-000924.

\section{Thierney 2007}

Tierney JF, Stewart LA, Ghersi D, Burdett S, Sydes MR. Practical methods for incorporating summary time-to-event data into meta-analysis. Trials 2007;8(1):16.

\section{Warner 2011}

Warner TD, Nylander S, Whatling C. Anti-platelet therapy: cyclooxygenase inhibition and the use of aspirin with particular regard to dual anti-platelet therapy. British Journal of Clinical Pharmacology 2011;72(4):619-33.

\section{Weitz 2016}

Weitz $\mathrm{JI}$, Jaffer IH. Optimizing the safety of treatment for venous thromboembolism in the era of direct oral anticoagulants. Polskie Archiwum Medycyny Wewnetrznej 2016;126(9):688-96.

\section{Wijeyeratne 2011}

Wijeyeratne YD, Heptinstall S. Anti-platelet therapy: ADP receptor antagonists. British Journal of Clinical Pharmacology 2011;72(4):647-57.

\section{Wilson 1999}

Wilson WA, Gharavi AE, Koike T, Lockshin MD, Branch DW, Piette JC, et al. International consensus statement on preliminary classification criteria for definite antiphospholipid syndrome: report of an international workshop. Arthritis and Rheumatism 1999;42(7):1309-11.

\section{References to other published versions of this review Bala 2016}

Bala MM, Celinska-Lowenhoff M, Padjas A, Szot W, Undas A. Antiplatelet and anticoagulant agents for secondary prevention of stroke and other thromboembolic events in people with antiphospholipid syndrome. Cochrane Database of Systematic Reviews 2016, Issue 4. Art. No: CD012169. [DOI: 10.1002/14651858.CD012169]

* Indicates the major publication for the study

\section{CHARACTERISTICS OF STUDIES}

Characteristics of included studies [ordered by study ID]

Crowther 2003

\section{Study characteristics}


Crowther 2003 (Continued)

Methods

Study type: double-blinded parallel RCT

Location: Canada, tertiary care rheumatology and thromboembolism clinics

Number of centres: 13

Time frame of the study: February 1998 to May 2001

Follow-up: mean 2.7 years (SD not provided)

\section{Participants}

Inclusion criteria: people with arterial/venous thrombosis, objectively confirmed; and either a positive test for antiphospholipid antibodies on 2 occasions $\geq 3$ months apart defined as lupus anticoagulant (according to the definition by the International Society on Thrombosis and Haemostasis), a moderate/high titre of IgG anticardiolipin antibody, or both

Exclusion criteria: anticardiolipin antibodies only in IgM class; clinically significant bleeding predisposition (e.g. refractory thrombocytopenia: platelet count $<50,000 / \mathrm{mm}^{3}$ ); an episode of intracranial hemorrhage, stroke, or gastrointestinal bleeding within the previous 3 months; a contraindication to warfarin; a history of recurrent thrombosis during warfarin treatment with a target INR $\geq 2.0$; pregnan$\mathrm{cy} /$ planned pregnancy during the study; a geographic location that would make follow-up impossible

Total number of participants: 114 participants randomized, 114 analyzed: 56 in intervention (highdose) group, 58 in control (standard-dose) group

\section{Characteristics}

Age (mean): high-dose group: 43 years (range 20 to 80); standard-dose group: 41 years (range 21 to 81 )

Sex: high-dose group: 27 (48\%) women; standard-dose group: 41 (72\%) women; baseline difference

Systemic lupus erythematosus: $14 \%$

Previous events: history of venous thrombosis: $75 \%$; thromboembolism within the last 6 months: $32 \%$

Cardiovascular risk factors: NR

Antibodies present: lupus anticoagulant only: 43\%; anticardiolipin antibodies only: 39\%; lupus anticoagulant and anticardiolipin antibodies: $18 \%$

Interventions

\section{Treatment groups}

- high-dose warfarin treatment with targeted INR of 3.1 to 4.0 ; average follow-up 2.6 years

- standard-dose warfarin treatment with targeted INR of 2.0 to 3.0; average follow-up 2.7 years

Average INR values in high-dose group: 3.3; in standard-dose group, 2.3

$\%$ of time when INR

- above the target: high: $17 \%$; standard: $11 \%$

- within the target: high: $40 \%$; standard: $71 \%$

- below the target: high: $43 \%$; standard: $19 \%$

In the high-dose group, INR was between 2.0 and 3.1 for $86 \%$ of time when below the target

Descriptions of treatments and concomitant treatment: 8 participants $(14 \%)$ in high-dose group and 6 participants (10\%) in standard-dose group received aspirin during the study

\section{Outcomes Primary outcomes}

- for treatment efficacy: recurrent trombosis (stroke, TIA, myocardial infarction, peripheral arterial thrombosis, cerebral vein thrombosis, deep vein thrombosis, or pulmonary embolism) - objective diagnostic tests, confirmed by blinded adjudication

Antiplatelet and anticoagulant agents for secondary prevention of stroke and other thromboembolic events in people with 
- for treatment safety: bleeding (major or any) - explicit definition not provided; objective diagnostic tests, confirmed by blinded adjudication

Secondary outcomes: not reported

\begin{tabular}{ll}
\hline Funding source & Canadian Institutes for Health Research; warfarin used in the study was provided by DuPont Pharma \\
\hline Notes & $\begin{array}{l}\text { Originally planned sample size was } 90 \text { participants, with minimum follow-up of } 2 \text { years. After blinded } \\
\text { interim analysis due to lower than expected number of events, the Steering Committee extended enrol- } \\
\text { ment and reduced the duration of follow-up; the last participants included to } 6 \text { months }\end{array}$ \\
\hline
\end{tabular}

\title{
Risk of bias
}

\begin{tabular}{|c|c|c|}
\hline Bias & Authors' judgement & Support for judgement \\
\hline $\begin{array}{l}\text { Random sequence genera- } \\
\text { tion (selection bias) }\end{array}$ & Low risk & Random numbers table: blocks of 2,4 , and 6 \\
\hline $\begin{array}{l}\text { Allocation concealment } \\
\text { (selection bias) }\end{array}$ & Low risk & Telephone calls to study coordinating centre \\
\hline $\begin{array}{l}\text { Blinding of participants } \\
\text { and personnel (perfor- } \\
\text { mance bias) } \\
\text { Obj. }\end{array}$ & Low risk & $\begin{array}{l}\text { Objective or objectively verified outcomes: thrombosis and bleeding: dou- } \\
\text { ble-blind; explicit statement that participants, treating physicians, other study } \\
\text { personnel unaware of the treatment assignments }\end{array}$ \\
\hline $\begin{array}{l}\text { Blinding of outcome as- } \\
\text { sessment (detection bias) } \\
\text { Obj. }\end{array}$ & Low risk & $\begin{array}{l}\text { Objective or objectively verified outcomes: thrombosis and bleeding: explicit } \\
\text { statement that adjudicators were unaware of the treatment assignment }\end{array}$ \\
\hline $\begin{array}{l}\text { Incomplete outcome data } \\
\text { (attrition bias) } \\
\text { All outcomes }\end{array}$ & Low risk & $\begin{array}{l}\text { Discontinuations, withdrawals, and missing data: } \\
\text { Intervention: } 21 / 56 \text { discontinued ( } 11 \text { withdrew consent; } 5 \text { suspected throm- } \\
\text { botic events; } 3 \text { major hemorrhage; } 1 \text { pregnant; } 1 \text { thrombocytopenia); } 4 / 56 \text { cen- } \\
\text { sored } \\
\text { Control: } 13 / 58 \text { discontinued ( } 7 \text { withdrew consent; } 5 \text { suspected thrombotic } \\
\text { event; } 1 \text { major hemorrhage): } 2 / 58 \text { censored } \\
\text { ITT: all participants included in the analysis; reasons for missing data reported, } \\
\text { unlikely to be related to study outcomes }\end{array}$ \\
\hline $\begin{array}{l}\text { Selective reporting (re- } \\
\text { porting bias) }\end{array}$ & Low risk & $\begin{array}{l}\text { No protocol available, but all thrombotic and bleeding outcomes pre-specified } \\
\text { and reported as stated in Methods section of published study }\end{array}$ \\
\hline Other bias & Low risk & None identified \\
\hline
\end{tabular}

Kondratyeva 2010

\section{Study characteristics}

Methods

\author{
Study type: open parallel RCT; no blinding \\ Location: Russia, specialist centre \\ Number of centres: 1 \\ Time frame of the study: 2003 to June 2008
}


Kondratyeva 2010 (Continued)

Follow-up: in combined treatment group 58.4 months (median; IQR 0.9; 38.9), in warfarin group follow-up was 51.6 months (median; IQR 0.9; 48.8)

\section{Participants}

Inclusion criteria: people with diagnosis of APS according to 2006 Miyakis criteria (information from the author)

Exclusion criteria: severe renal or hepatic insufficiency, severe bleeding in the last 3 months, planning pregnancy in the next year; severe thrombocytopenia $<50000$

Total number of participants: 82 randomized and analyzed: 49 into warfarin group and 33 into aspirin and warfarin group (information confirmed with the authors)

Characteristics: for warfarin/combined group

Mean age: $36.4(11.2) / 40.5$ years (SD 11.9)

Women: $73 \% / 76 \%$

Systemic lupus erythematosus: $56.9 \% / 51.5 \%$

Previous events: VTE 65.3\%/78.8\%; arterial thrombosis 48.9\%/54.5\%

\section{Treatment groups}

- warfarin-adjusted dose with a target INR 2.0 to 3.0 and aspirin $100 \mathrm{mg} / \mathrm{d}$

- warfarin-adjusted dose with a target INR 2.0 to 3.0

\section{Descriptions of treatments and concomitant treatment: NR}

\section{Outcomes}

Outcomes: recurrence of thrombosis (instrumental verification for thrombotic events: ultrasound duplex scanning of blood vessels; CT of the lungs; clinical symptoms of acute cerebral ischemia confirmed by CT or MRI of the brain; information from the author)

TIA (diagnosed by neurologist, transcranial dopplerography of cerebral arteries for changes in neurological status for $>6$ hours, CT or MRI in diagnostically unclear cases), hemorrhage major (fatal or life threatening or resulting in lowering of hemoglobin level requiring transfusion or hospitalization), hemorrhage minor (not requiring medical attention, such as nose bleeding, microhematuria, bleeding from the gums, bruising) recorded by the participants in their diaries

Funding source NR

Notes The study was published in 2 parts: in the 2007 publication it reported on a total of 60 participants, of whom 50 were randomized (on the basis of the information from the author), while 10 were assigned to warfarin-only group due to thrombocytopenia or minor bleeding) and in the 2010 publication the study included an additional 32 randomized participants making a total of 82 randomized

In the final publication, no information on APS criteria used for diagnosis

\section{Risk of bias}

\begin{tabular}{lll}
\hline Bias & Authors' judgement & Support for judgement \\
\hline $\begin{array}{l}\text { Random sequence genera- } \\
\text { tion (selection bias) }\end{array}$ & Low risk & $\begin{array}{l}\text { Simple randomization - coin tossing - "heads" for monotherapy and "tails" for } \\
\text { combination group (information from the author) }\end{array}$ \\
\hline $\begin{array}{l}\text { Allocation concealment } \\
\text { (selection bias) }\end{array}$ & High risk & $\begin{array}{l}\text { Doctor who was tossing coin was aware of group assignment (information } \\
\text { from the author) }\end{array}$ \\
\hline $\begin{array}{l}\text { Blinding of participants } \\
\text { and personnel (perfor- } \\
\text { mance bias) }\end{array}$ & Low risk & $\begin{array}{l}\text { Objective or objectively verified outcomes: thrombosis and major bleeding: no } \\
\text { blinding, outcome unlikely to be influenced by the lack of blinding }\end{array}$ \\
\hline
\end{tabular}


Kondratyeva 2010 (Continued)

Obj.

\begin{tabular}{|c|c|c|}
\hline $\begin{array}{l}\text { Blinding of participants } \\
\text { and personnel (perfor- }\end{array}$ & High risk & $\begin{array}{l}\text { Self-reported outcomes: minor bleeding: no blinding, self-reported outcome } \\
\text { likely to be influenced by the lack of blinding }\end{array}$ \\
\hline
\end{tabular}
mance bias)

Subj.

\begin{tabular}{|c|c|c|}
\hline $\begin{array}{l}\text { Blinding of outcome as- } \\
\text { sessment (detection bias) } \\
\text { Obj. }\end{array}$ & Low risk & $\begin{array}{l}\text { Objective or objectively verified outcomes: thrombosis and major bleeding: no } \\
\text { blinding, outcome unlikely to be influenced by the lack of blinding }\end{array}$ \\
\hline $\begin{array}{l}\text { Blinding of outcome as- } \\
\text { sessment (detection bias) } \\
\text { Subj. }\end{array}$ & High risk & $\begin{array}{l}\text { Self-reported outcomes: minor bleeding: no blinding, self-reported outcome, } \\
\text { likely to be influenced by the lack of blinding }\end{array}$ \\
\hline $\begin{array}{l}\text { Incomplete outcome data } \\
\text { (attrition bias) } \\
\text { All outcomes }\end{array}$ & Low risk & $\begin{array}{l}\text { Additional information obtained from the authors: all patients randomized } \\
\text { were analyzed }\end{array}$ \\
\hline $\begin{array}{l}\text { Selective reporting (re- } \\
\text { porting bias) }\end{array}$ & Low risk & $\begin{array}{l}\text { No protocol available, but all thrombotic and bleeding outcomes pre-specified } \\
\text { and reported as stated in Methods section of published study }\end{array}$ \\
\hline Other bias & Unclear risk & Insufficient information \\
\hline
\end{tabular}

\section{Okuma 2010}

\section{Study characteristics}

Methods Study type: double-blinded parallel RCT

Location: Japan, Departments of Neurology of University Hospitals

\section{Number of centres: NR}

Time frame of the study: October 2002 to November 2004

Follow-up: mean 3.9 years (SD 2.0)

Participants

Inclusion criteria: participants with history of ischemic stroke; antiphospholipid antibodies on 2 or more occasions $\geq 6$ weeks apart: positive IgG beta 2 glycoprotein I $\left(\beta_{2}\right.$-GPI)-dependent anticardiolipin antibody and/or lupus anticoagulant present

\section{Exclusion criteria: NR}

Total number of participants: 20 participants randomized, 20 analyzed: 11 in single AP group (AP), 9 in AP + VKA group

\section{Characteristics}

Mean age: AP: 47 years; AP + VKA: 49 years

Sex: $50 \%$ women

Systemic lupus erythematosus: $35 \%$

Previous events: stroke: $100 \%$

Cardiovascular risk factors: hypertension $59.6 \%$, diabetes mellitus $20.2 \%$, atrial fibrillation $10.1 \%$, hyperlipidemia $20.2 \%$ 
Okuma 2010 (Continued)

Antibodies present: NR

\begin{tabular}{|c|c|}
\hline Interventions & $\begin{array}{l}\text { Treatment groups } \\
\text { - single antiplatelet therapy ( } 100 \mathrm{mg} \text { aspirin) } \\
\text { - combination of antiplatelet and anticoagulant treatment (a target INR of } 2 \text { to 3); mean INR } 2.4 \text { (SD 0.3) } \\
\text { Descriptions of treatments and concomitant treatment: no concomitant treatment reported }\end{array}$ \\
\hline Outcomes & $\begin{array}{l}\text { Primary outcomes: recurrent episode of stroke - no definition or method of verification provided } \\
\text { Secondary outcomes: hemorrhagic complications (e.g. cerebral hemorrhage, gastrointestinal bleed- } \\
\text { ing, subcutaneous hemorrhage)-no definitions or method of verification provided }\end{array}$ \\
\hline Funding source & NR \\
\hline Notes & $\begin{array}{l}\text { No details of sample size calculations provided; several attempts to contact the authors for additional } \\
\text { information unsuccessful }\end{array}$ \\
\hline
\end{tabular}

\title{
Risk of bias
}

\begin{tabular}{|c|c|c|}
\hline Bias & Authors' judgement & Support for judgement \\
\hline $\begin{array}{l}\text { Random sequence genera- } \\
\text { tion (selection bias) }\end{array}$ & Unclear risk & $\begin{array}{l}\text { Insufficient information to judge; the authors stated that randomization was } \\
\text { done using "a randomly generated score" }\end{array}$ \\
\hline $\begin{array}{l}\text { Allocation concealment } \\
\text { (selection bias) }\end{array}$ & Unclear risk & Insufficient information to judge \\
\hline $\begin{array}{l}\text { Blinding of participants } \\
\text { and personnel (perfor- } \\
\text { mance bias) } \\
\text { Obj. }\end{array}$ & Unclear risk & $\begin{array}{l}\text { Objective or objectively verified outcomes: stroke and hemorrhage: described } \\
\text { as double-blind, but blinding unclear; no information about definition or ob- } \\
\text { jective outcome verification so influence of lack of blinding not clear }\end{array}$ \\
\hline $\begin{array}{l}\text { Blinding of outcome as- } \\
\text { sessment (detection bias) } \\
\text { Obj. }\end{array}$ & Unclear risk & $\begin{array}{l}\text { Objective or objectively verified outcomes: stroke and hemorrhage: described } \\
\text { as double-blind, but blinding unclear; no information about definition of ob- } \\
\text { jective outcome verification so influence of lack of blinding not clear }\end{array}$ \\
\hline $\begin{array}{l}\text { Incomplete outcome data } \\
\text { (attrition bias) } \\
\text { All outcomes }\end{array}$ & Low risk & No discontinuations or withdrawals reported; all outcome data included \\
\hline $\begin{array}{l}\text { Selective reporting (re- } \\
\text { porting bias) }\end{array}$ & Unclear risk & $\begin{array}{l}\text { No protocol available, primary outcomes partially reported as indicated in } \\
\text { Methods section of published study, but the numbers of events not reported }\end{array}$ \\
\hline Other bias & Low risk & None identified \\
\hline
\end{tabular}

Ordi-Ros 2019

\section{Study characteristics}

Methods

\author{
Study type: multicenter, phase 3, parallel, open-label, non-inferiority RCT \\ Location: Spain \\ Number of centres: 6 (hospitals)
}


Time frame of the study: recruitment from March 2013 to December 2014. Follow-up completed in December 2017

Follow-up: overall mean follow-up was 35.4 months (range from 18 to 36 months)

Participants

Inclusion criteria: adult men and women (age 18 to 75 years); fulfilling the international consensus clinical criteria for APS (Miyakis 2006 criteria (Sydney)) - history of thrombosis with or without pregnancy morbidity. In case of newly diagnosed APS there must have been stable disease and treatment with VKAs for at least 6 months; positive for any of listed in the brackets antiphospholipid antibodies at the baseline blood sample (anticardiolipin antibodies $(\mathrm{aCL})$ : moderate or high positive IgG/M ( $\geq 40 \mathrm{GPL}$ or MPL units or > 99th percentile); anti-beta2-glycoprotein I (anti- $32 \mathrm{GPI}$ ) antibodies: moderate or high positive IgG/M ( $\geq 40 \mathrm{GPL}$ or MPL units or $>99$ th percentile); lupus anticoagulant (LA) (based on the Recommendations from International Society of Thrombosis and Haemostasis); in women of childbearing potential (not undergone sterilization and not postmenopausal) - effective method of birth control during the study up to 3 months after the last dose of study drug (hormonal contraception or barrier method)

Exclusion criteria: Increased risk of hemorrhage: bleeding diathesis (such as refractory thrombocytopenia with platelets $\leq 50 \times 10^{9}$ cells/L); previous bleeding events (intracranial hemorrhage, stroke, gastrointestinal bleeding in the last 3 months before the study); pregnancy or lactation; severe renal (creatinine clearance $\leq 30 \mathrm{~mL} / \mathrm{min} / 1.73 \mathrm{~m} 2$ ) or liver function impairment (alanine aminotransferase level $>2 x$ the upper limit of normal); liver cirrhosis with Child-Pugh class B or C; not adherent to warfarin treatment; receiving drugs which induce cytochrome P450 3A4

Total number of participants: 190

- number of participants randomized: rivaroxaban: 95, VKA (acenocoumarol): 95

- number of participants included in the analysis: rivaroxaban: 80, VKA: 87

\section{Characteristics}

- age (median [IQR]): rivaroxaban: 47 (40-55), VKA: 51 (38-63)

- sex (women N [\%]): rivaroxaban: 61 (64.2\%), VKA: 60 (63.2\%)

- BMI (mean [SD]0]: rivaroxaban: 28 (5.1), VKA: 29 (6.0)

- previous events (criteria for initial anticoagulation) (N [\%]):

* arterial: rivaroxaban: 37 (38.9\%), VKA: $34(35.8 \%)$

* venous: rivaroxaban: 69 (72.6\%), VKA: 70 (73.7\%)

* both: rivaroxaban: 11 (11.6\%), VKA: 9 (9.5\%)

- SLE (N [\%]): rivaroxaban: 31 (32.6\%), VKA: 27 (28.4\%)

- presence of LA (N [\%]): rivaroxaban: 93 (97.9\%), VKA: 90 (94.7\%)

- presence of anticardiolipin AB (N [\%]): rivaroxaban: 62 (65.3\%), VKA: 65 (68.4\%)

- presence of AntiB-2glycoprotein I AB (N [\%]): rivaroxaban: 60 (63.2\%), VKA: 62 (65.3\%)

- triple positive antiphospholipid antibodies (N [\%]): rivaroxaban: 58 (61.1\%), VKA: 57 (60\%)

\section{Comorbidities}

- hypertension (N [\%]): rivaroxaban: 39 (41.1\%), VKA: 38 (40.0\%)

- diabetes (N [\%]): rivaroxaban: 8 (8.4\%), VKA: 4 (4.2\%)

- dyslipidemia (N [\%]): rivaroxaban: 40 (42.1\%), VKA: 34 (35.8\%)

- smoking (N [\%]): rivaroxaban: 28 (29.5\%), VKA: 38 (40.0\%)

\section{Treatment groups}

- rivaroxaban $20 \mathrm{mg} / \mathrm{d}$ or $15 \mathrm{mg} / \mathrm{d}$, according to renal function (participants with creatine clearance 30-49 L/min - $15 \mathrm{mg} / \mathrm{d}$ )

- dose-adjusted VKAs (acenocoumarol) with INR controlled every 2 to 6 weeks - a target INR of 2.0 to 3.0 , or 3.1 to 4.0 in participants with a history of recurrent thrombosis

\section{Descriptions of treatments and concomitant treatment}


- rivaroxaban group (N [\%]): aspirin use - 13 (13.7\%), beta-blockers - 6 (6.3\%), diuretics - 2 (2.1\%), ACEI - $38(40.0 \%)$, Ca-antagonists - 8 (8.4\%), statins - 40 (42.1\%), oral steroids - $26(27.4 \%)$, anti-malarials 29 (30.5\%), immunosuppression - 19 (20.0\%)

- VKA group (N [\%]): aspirin use - 11 (11.6\%), beta-blockers - 9 (9.5\%), diuretics - 1 (1.1\%), ACEI - 36 $(37.9 \%)$, Ca-antagonists - $6(6.3 \%)$, statins $-34(35.8 \%)$, oral steroids - $23(24.2 \%)$, anti-malarials - 26 (27.4\%), immunosuppression - 20 (21.1\%)

\begin{tabular}{ll}
\hline Outcomes & Primary outcomes: new thrombotic events, major bleeding \\
Secondary outcomes: time to thrombosis, type of thrombosis (arterial or venous), changes in bio- \\
marker levels (D-dimer, von Willebrand factor, and platelet factor 4), cardiovascular death, non-major \\
bleeding, any adverse event
\end{tabular}

Funding source

Bayer Hispania - Authors report: "Funding source - Bayer Hispania - Bayer Hispania had no role in the design or conduct of the study; collection, management, analysis, or interpretation of the data; or preparation, review, or approval of the manuscript." However; 2 authors reported links with commercial entities

Notes Online protocols: EudraCT 2010-019764-36; NCT02926170

\section{Risk of bias}

\begin{tabular}{|c|c|c|}
\hline Bias & Authors' judgement & Support for judgement \\
\hline $\begin{array}{l}\text { Random sequence genera- } \\
\text { tion (selection bias) }\end{array}$ & Low risk & $\begin{array}{l}\text { Computer-generated random-number sequences (C4-Study design pack soft- } \\
\text { ware [Glaxo-SmithKline]) in blocks of } 10\end{array}$ \\
\hline $\begin{array}{l}\text { Allocation concealment } \\
\text { (selection bias) }\end{array}$ & Low risk & $\begin{array}{l}\text { Sequentially-numbered, concealed envelopes containing group assignments } \\
\text { - after written informed consent was obtained, the envelopes were opened in } \\
\text { sequence and participants were randomly assigned in a 1:1 ratio }\end{array}$ \\
\hline $\begin{array}{l}\text { Blinding of participants } \\
\text { and personnel (perfor- } \\
\text { mance bias) } \\
\text { Obj. }\end{array}$ & Low risk & $\begin{array}{l}\text { Objective or objectively verified outcomes: new thrombotic events, major } \\
\text { bleeding, time to thrombosis, type of thrombosis (arterial or venous), changes } \\
\text { in biomarker levels, cardiovascular death, non-major bleeding: the lack of } \\
\text { blinding will likely not influence the outcomes }\end{array}$ \\
\hline $\begin{array}{l}\text { Blinding of participants } \\
\text { and personnel (perfor- } \\
\text { mance bias) } \\
\text { Subj. }\end{array}$ & High risk & $\begin{array}{l}\text { Self-reported outcomes: any adverse events: likely to be influenced by the lack } \\
\text { of blinding }\end{array}$ \\
\hline $\begin{array}{l}\text { Blinding of outcome as- } \\
\text { sessment (detection bias) } \\
\text { Obj. }\end{array}$ & Low risk & $\begin{array}{l}\text { New thrombotic events, major bleeding, time to thrombosis, type of throm- } \\
\text { bosis (arterial or venous), changes in biomarker levels, cardiovascular death, } \\
\text { non-major bleeding: "an independent committee blinded to the clinical end } \\
\text { points applied protocol definitions to adjudicate suspected cases of throm- } \\
\text { bosis, death, and bleeding events that contributed to the prespecified end } \\
\text { points" }\end{array}$ \\
\hline
\end{tabular}

\begin{tabular}{|c|c|c|}
\hline $\begin{array}{l}\text { Blinding of outcome as- } \\
\text { sessment (detection bias) } \\
\text { Subj. }\end{array}$ & Low risk & $\begin{array}{l}\text { Self reported outcomes: any adverse events: "All adverse events were re- } \\
\text { viewed by an independent committee and classified into serious and minor" - } \\
\text { information from authors }\end{array}$ \\
\hline $\begin{array}{l}\text { Incomplete outcome data } \\
\text { (attrition bias) } \\
\text { All outcomes }\end{array}$ & Low risk & $\begin{array}{l}\text { Outcome data complete in both groups. Both PP and ITT analysis reported. In } \\
\text { both groups deaths, early discontinuations including adverse events reported: } \\
\text { - rivaroxaban: death }(n=5) \text {; early discontinuation }(n=6) \text { (lupus nephritis flare: } \\
\text { 1, adverse events: } 5) \\
\text { - VKA: death }(n=3) \text {; early discontinuation }(n=6) \text { (lupus nephritis flare: } 1 \text {, ad- } \\
\text { verse events: } 1 \text {, irregular INR control: } 4)\end{array}$ \\
\hline
\end{tabular}


Ordi-Ros 2019 (Continued)

In each group, $6.3 \%$ of participants permanently stopped their assigned therapy before a thrombotic event and before the end date

\begin{tabular}{lll}
\hline $\begin{array}{l}\text { Selective reporting (re- } \\
\text { porting bias) }\end{array}$ & Low risk & $\begin{array}{l}\text { Protocol reported both as appendix and registered online. All prespecified out- } \\
\text { comes reported }\end{array}$ \\
\hline Other bias & Unclear risk & It was not possible to assess whether the trial was free of other biases \\
\hline
\end{tabular}

\section{RAPS 2016}

\section{Study characteristics}

Study type: unblinded phase II/III non-inferiority RCT
Location: UK, specialist hematology and rheumatology clinics
Number of centres: 2
Time frame of the study: June 2013 to November 2014
Follow-up: 210 days

Participants

Inclusion criteria: thrombotic APS according to Sapporo criteria with positive antiphospholipid antibodies (lupus anticoagulant, IgG or IgM anticardiolipin or anti- $\beta_{2}$-GPI antibodies above 99th percentile) on $\geq 2$ occasions $\geq 12$ weeks apart; $\geq 1$ episode of VTE during sub-therapeutic (INR $<2.0$ ) anticoagulant treatment or no treatment; on standard dose warfarin (a target INR of 2.5) for $\geq 3$ months since the last VTE; contraception in women (unless sterilized or postmenopausal)

Exclusion criteria: previous arterial thrombotic events due to APS; recurrent VTE on warfarin at a target INR of 2 to 3; age < 18 years; pregnancy or lactation; severe renal impairment (creatinine clearance using Cockcroft and Gault formula $\leq 29 \mathrm{~mL} / \mathrm{min}$ ); alanine aminotransferase $>2$ upper limit of normal; cirrhosis of Child-Pugh class B or C; thrombocytopenia $\left(<75 \times 10^{9} / \mathrm{L}\right)$; non-adherence to warfarin regimen according to clinical judgment; receiving drugs: azole antifungals, protease inhibitors for HIV, strong CYP3A4 inducers, dronedarone; refusal to provide information to a family doctor or other health-care professional responsible for anticoagulation care about study participation

Total number of participants: 116 participants randomized: 57 in the rivaroxaban group and 50 in the warfarin group; 110 analyzed for the primary outcome (thrombin potential endogenous); for outcomes analyzed in this review: 57 in the rivaroxaban group, 58 for efficacy, and 55 or 58 for safety in the warfarin group

Characteristics: no baseline differences reported

Mean age: rivaroxaban 47 years (SD 17); warfarin 50 years (SD 14)

BMI: rivaroxaban 28 (SD 6); warfarin 30 (SD 6)

INR: rivaroxaban 2.8 ( $95 \% \mathrm{Cl} 2.6$ to 2.9$)$; warfarin 2.7 (95\% Cl 2.5 to 3.0$)$

Sex: $72 \%$ women

Systemic lupus erythematosus: $19 \%$

Previous events: deep vein thrombosis or pulmonary embolism $100 \%$

Cardiovascular risk factors: NR

Antibodies present: lupus anticoagulant only: $46 \%$; anticardiolipin antibodies only: $3 \% ; \beta_{2}$-GPI antibodies only: 4\%; more than 1 type of antibodies without triple positive: 30\%; more than 1 type of antibodies including triple positive: $16 \%$ 
RAPS 2016 (Continued)

\author{
Interventions

\section{Treatment groups} \\ - rivaroxaban \\ - warfarin at a target INR of 2.5; at day 42; INR 2.7 (95\% CI 2.6 to 2.9 ) \\ Participants received interventions for 180 days \\ Mean INR in warfarin group: 2.7 (95\% Cl 2.6 to 2.9 ). Mean time in therapeutic range $55 \%$ (SD 23 )
}

Descriptions of treatments and concomitant treatment: concomitant treatment not reported

Primary outcomes
Change (percentage) in endogenous thrombin potential from randomization to day 42 of study
Secondary outcomes
Thromboembolism up to day 210 (in the protocol 180 days) -VTE only or VTE and any other thrombotic
events-verification by objective diagnostic methods
Percentage change up to day 42: thrombin generation curve (lag-time, time to peak, peak thrombin
concentration); markers of in vivo coagulation (prothrombin fragment 1.2, thrombin-antithrombin
complex, D-dimer)

SAEs to day 210 - reviewed by external independent staff

Bleeding events to day 210 - blinded review of bleeding events

Quality of life at day 180 - measured using the EQ-5D-5L questionnaire

Funding source

Arthritis Research UK; Comprehensive Clinical Trials Unit at UCL; LUPUS UK, Bayer; National Institute for Health Research Biomedical Research Centre

\title{
Notes
}

Protocol: ISRCTN68222801; NCT02116036; EudtraCT 2012-002345-38

\section{Risk of bias}

\begin{tabular}{|c|c|c|}
\hline Bias & Authors' judgement & Support for judgement \\
\hline $\begin{array}{l}\text { Random sequence genera- } \\
\text { tion (selection bias) }\end{array}$ & Low risk & Random permuted blocks of various length, stratified \\
\hline $\begin{array}{l}\text { Allocation concealment } \\
\text { (selection bias) }\end{array}$ & Low risk & Central \\
\hline $\begin{array}{l}\text { Blinding of participants } \\
\text { and personnel (perfor- } \\
\text { mance bias) } \\
\text { Obj. }\end{array}$ & Low risk & $\begin{array}{l}\text { Objective or objectively verified outcomes: thromboembolism and laborato- } \\
\text { ry coagulation measures, SAE, bleeding: due to objective definition or verifica- } \\
\text { tion of outcomes, lack of blinding will likely not influence outcomes }\end{array}$ \\
\hline $\begin{array}{l}\text { Blinding of participants } \\
\text { and personnel (perfor- } \\
\text { mance bias) } \\
\text { Subj. }\end{array}$ & High risk & Subjective outcome: quality of life, lack of blinding likely introduces bias \\
\hline $\begin{array}{l}\text { Blinding of outcome as- } \\
\text { sessment (detection bias) } \\
\text { Obj. }\end{array}$ & Low risk & $\begin{array}{l}\text { Objective or objectively verified outcomes: thromboembolism and laborato- } \\
\text { ry coagulation measures: SAE - due to objective definition/verification of out- } \\
\text { comes lack of blinding will likely not influence outcomes; Bleeding: blinded } \\
\text { verification of outcomes }\end{array}$ \\
\hline
\end{tabular}

Antiplatelet and anticoagulant agents for secondary prevention of stroke and other thromboembolic events in people with antiphospholipid syndrome (Review)

Copyright @ 2020 The Cochrane Collaboration. Published by John Wiley \& Sons, Ltd. 
RAPS 2016 (Continued)

Blinding of outcome as

High risk Subjective outcome: quality of life, lack of blinding likely introduces bias sessment (detection bias)

Subj.
Incomplete outcome data Low risk (attrition bias)

All outcomes
Discontinuations, withdrawals and missing data:

intervention: $1 / 57$ with missing data - did not attend 180-day visit, included in analyses of thrombotic and bleeding event, excluded from quality of life analyses

control: 4/59 with missing data ( 1 withdrew consent and no follow-up data obtained; 1 withdrew consent after day 42 but returned for 180-day visit; 1 died after day 180; 1 lost to follow-up after day 42); 1 excluded from analysis of thrombotic events; 4 excluded from analysis of bleeding events at day 210; 3 to 4 excluded from analyses of quality of life data

Small amount of missing data; missing outcome data balanced in numbers across intervention groups, with similar reasons for missing data across groups; modified ITT analysis including all randomized participants with assessable data

Selective reporting (re- Low risk All reported as in protocol
porting bias)
All reported as in protocol

None identified

\section{Study characteristics}

Methods

Other bias Low risk None identified

\section{Methods}

Study type: non-inferiority, phase 3, parallel, open-label RCT

Location: Italy

Number of centres: approximately 40

Time frame of the study: December 2014 to December 2018

Follow-up: 611 days for ITT, 569 days for "as treated"; study terminated prematurely due to excess of events in rivaroxaban group

\section{Participants}

Inclusion criteria: adults (age 18 to 75 years); confirmed APS diagnosis: history of thrombosis with or without pregnancy morbidity (Miyakis criteria) and positive for all three types of antiphospholipid antibodies (IgG or IgM aCL (> $40 \mathrm{GPL}$ or MPL or $>99$ th percentile), IgG or IgM anti- $\beta_{2}-\mathrm{GPI}$ (> $40 \mathrm{U}$ or $>99$ th percentile), lupus anticoagulant (test according to the Recommendations from the International Society of Thrombosis and Hemostasis).

Exclusion criteria: rivaroxaban-related severe hyperreactivity; creatinine clearance $<30 \mathrm{~mL} / \mathrm{min}$; pregnancy or breastfeeding at the time of inclusion; concomitant treatment with other anticoagulants or with drugs which inhibit p-glycoprotein and CYP3A4 inhibitors; procedure or conditions associated with hemorrhage: major surgical procedure/trauma up to 30 days before the study; clinically significant GI bleeding within 6 months before randomization; history of intracranial, intraocular, spinal or atraumatic intra-articular bleeding; chronic hemorrhagic disorder; intracranial neoplasm, arteriovenous malformation or aneurysm; scheduled invasive procedure with possibility of uncontrolled bleeding; systolic blood pressure $180 \mathrm{mmHg}$ or higher; liver cirrhosis or ALT > 3 upper normal value

Total number of participants: planned sample size 536 participants, finally terminated after including 120; 
TRAPS 2016 (Continued)

\section{Characteristics}

- $\operatorname{sex}$ (women N[\%]): rivaroxaban: 39 (66\%), VKA: 38 (62\%)

- age (mean [SD]): rivaroxaban: 46.5 (10.2), VKA: 46.1 (13.2)

- BMI (mean [SD]): rivaroxaban: 26.1 (6.1), VKA: 25.5 (5.9)

- previous events (N [\%]):

* arterial: rivaroxaban: 11 (19\%), VKA: $14(23 \%)$

* venous: rivaroxaban: 38 (64\%), VKA: 39 (64\%)

* both: rivaroxaban: 10 (17\%), VKA: 8 (13\%)

* pregnancy morbidity in women: rivaroxaban: $16(41 \%)$, VKA: $12(32 \%)$

- SLE (N [\%]): rivaroxaban: 10 (17\%), VKA: 15 (25\%)

- presence of LA (diluted Russel viper venom/activated partial thromboplastin time/both; N): rivaroxaban: 16/5/38, VKA: 14/7/40

- presence of anticardiolipin AB (IgG or IgG and IgM/only IgM; N): rivaroxaban: 57/2, VKA: 52/9

- presence of antiB-2-glycoprotein IAB (IgG or IgG and IgM/only IgM; N): rivaroxaban: 57/2, VKA: 52/9

\section{Comorbidities}

- smoking (N [\%]): rivaroxaban: 31 (53\%), VKA: 29 (48\%)

- hypertension (N [\%]): rivaroxaban: $15(25 \%)$, VKA: $22(36 \%)$

- diabetes (N [\%]): rivaroxaban: 4 (7\%), VKA: 0

- dyslipidemia (N [\%]): rivaroxaban: 12 (20\%), VKA: 15 (25\%)

- other condition with increased tendency for coagulation (N [\%]): rivaroxaban: 9 (15\%), VKA: 9 (15\%)

$\begin{array}{ll}\text { Interventions } & \text { Treatment groups } \\ \text { - } & \text { rivaroxaban } 20 \mathrm{mg} \text { daily, or } 15 \mathrm{mg} \text { daily if } \mathrm{CrCl}=30-50 \mathrm{~mL} / \mathrm{min} \\ \text { - } & \text { warfarin at a target INR of } 2.0 \text { to } 3.0\end{array}$

\section{Descriptions of treatments and concomitant treatment}

- Rivaroxaban group (n[\%]): hydroxychloroquine - 15 (25), corticosteroids - 11 (19), other immunosuppressive drugs - 17 (29), aspirin - 11 (19), statins - 7 (12)

- Warfarin group ( $[\%[\%])$ : hydroxychloroquine - 23 (38), corticosteroids - 13 (21), other immunosuppressive drugs - 21 (34), aspirin - 10 (16), statins - 10 (16)

$\begin{array}{ll}\text { Outcomes } & \text { Primary outcome: composite outcome including thromboembolic events, major bleeding or death } \\ \text { Secondary outcome: (efficacy) each type of thromboembolic event separately, all-cause mortality; } \\ \text { (safety) major/minor bleeding }\end{array}$

\begin{tabular}{|c|c|c|}
\hline Funding source & \multicolumn{2}{|c|}{ Supported by funding from Bayer S.p.A } \\
\hline Notes & \multicolumn{2}{|c|}{ Protocol: NCT02157272; EudraCT 2013-004575-13 } \\
\hline \multicolumn{3}{|l|}{ Risk of bias } \\
\hline Bias & Authors' judgement & Support for judgement \\
\hline $\begin{array}{l}\text { Random sequence genera- } \\
\text { tion (selection bias) }\end{array}$ & Low risk & $\begin{array}{l}\text { Web-based management system (Research Electronic Data Capture) - random- } \\
\text { ization 1:1 with random blocks of } 2,4 \text { and } 6 \text {, stratified (sex, presence of autoim- } \\
\text { mune disease) }\end{array}$ \\
\hline $\begin{array}{l}\text { Allocation concealment } \\
\text { (selection bias) }\end{array}$ & Low risk & $\begin{array}{l}\text { Central randomization using web-based management system (Research Elec- } \\
\text { tronic Data Capture) }\end{array}$ \\
\hline
\end{tabular}


TRAPS 2016 (Continued)

Blinding of participants Low risk Objective or objectively verified outcomes: thromboembolism, bleeding, morand personnel (perfortality: lack of blinding will likely not influence the outcomes mance bias)

Obj.

Blinding of participants Unclear risk Insufficient details to judge for any adverse events
and personnel (perfor-
mance bias)
Subj.

\begin{tabular}{|c|c|c|}
\hline $\begin{array}{l}\text { Blinding of outcome as- } \\
\text { sessment (detection bias) } \\
\text { Obj. }\end{array}$ & Low risk & $\begin{array}{l}\text { Objective or objectively verified outcomes: outcome assessors were blinded } \\
\text { (all outcomes were reviewed by independent adjudication committee, not } \\
\text { aware of treatment allocation) }\end{array}$ \\
\hline
\end{tabular}

Blinding of outcome as- Unclear risk Insufficient details to judge for any adverse events

sessment (detection bias)

Subj.

\section{Incomplete outcome data Low risk} (attrition bias)

All outcomes

\begin{abstract}
Discontinuations, withdrawals and missing data:
Reasons for discontinuation during the study period were reported:
\end{abstract}

rivaroxaban: 9 participants discontinued: due to withdrawal of informed consent (2), clinically-relevant non-major bleeding (2), planned pregnancy (3), other (2)

VKA group: 3 participants discontinued: due to withdrawal of informed consent (1), planned pregnancy (1), other (1)

VKA group: primary analysis "as treated" (participants who completed the study on treatment assigned at randomization) but ITT analysis reported

\begin{tabular}{|c|c|c|}
\hline $\begin{array}{l}\text { Selective reporting (re- } \\
\text { porting bias) }\end{array}$ & High risk & $\begin{array}{l}\text { Primary outcomes reported as specified in the protocol, also with details re- } \\
\text { garding each type of event. The protocol specified minor bleeding and adverse } \\
\text { events, but did not report the results }\end{array}$ \\
\hline \multirow[t]{2}{*}{ Other bias } & High risk & $\begin{array}{l}\text { The study planned to enroll } 536 \text { participants, however it was terminated after } \\
\text { including } 120 \text { participants }\end{array}$ \\
\hline & & $\begin{array}{l}\text { Insufficient information, not full report of the results; compliance: adherence } \\
\text { to treatment was } 96 \% \text { in the rivaroxaban group, and the time in therapeutic } \\
\text { range was } 67 \% \text { in the warfarin group }\end{array}$ \\
\hline
\end{tabular}

Location: hematological centres in Italy, Norway, Poland, Argentina, Czech Republic, and Slovak Republic

\section{Number of centres: 26}

\section{Time frame of the study: NR}

Follow-up: median 3.6 years (IQR 2.7 to 4.5); mean high dose group 3.5 (SD 1.2); standard management group 3.3 (SD 1.2); mean for both groups 3.4 (SD 1.2) 
WAPS 2005 (Continued)

Participants
Inclusion criteria: people with antiphospholipid syndrome diagnosed within previous 5 years (all had confirmed history of major arterial or venous thrombosis) for whom clinicians were uncertain regarding benefit/risk balance of high dose warfarin

Exclusion criteria: age $<18$; recurrent thrombosis during anticoagulant prophylaxis in past; active bleeding or hemorrhagic disorders contraindicating oral anticoagulant therapy; pregnancy; co-morbidities precluding oral anticoagulants or any serious illness with a life expectancy < 3 years; inability to give informed consent or to attend regular follow-up visits; evident benefit of high dose warfarin (e.g. recurrent thrombosis despite treatment with low-dose warfarin); platelets $<50 \times 10^{9} / \mathrm{L}$; hypothrombinemia/LA hemorrhagic syndrome; acute viral and HIV infection

Total number of participants: 109 participants randomized, although 6 participants were not followed: 109 analyzed: 54 in high dose group, 55 in standard-management group

\section{Characteristics}

Mean age: high-dose warfarin group: 41.1 years (SD 12.1); standard-management group: 41.0 years (SD 12.3)

Sex: $62 \%$ women

Systemic lupus erythematosus: $13 \%$

Previous events: prior arterial thrombosis: $40 \%$, prior venous thrombosis: $69 \%$

Cardiovascular risk factors: NR

Antibodies present: lupus anticoagulant only: $26 \%$, anticardiolipin antibodies only: $18 \%$, lupus anticoagulant and anticardiolipin antibodies: $56 \%$

Data regarding antiphospholipid antibodies reported for 52/54 participants in the high dose group and $52 / 55$ participants in the standard management group as other participants had borderline values for antibodies (information from the authors)

\section{Treatment groups}

- high-dose warfarin treatment with INR range 3.0 to 4.5 , a target of 3.5

- standard management, which included:

* warfarin at doses adjusted to an INR 2.0 to 3.0, a target of 2.5 (in participants with history of VTE, cardioembolic cerebral or peripheral ischemias, AF or rheumatic valve disease) (52 participants), or

* low-dose aspirin $100 \mathrm{mg} / \mathrm{d}$ (participants with non-embolic arterial thrombosis) (3 participants-

Mean INR during follow up 3.2 (SD 0.6) in high dose group and 2.5 (SD 0.3) in standard group

\section{Descriptions of treatments and concomitant treatment}

4 participants $(7.4 \%)$ in the high-dose warfarin group and 3 participants $(5.5 \%)$ in standard-management group were given anticoagulation + aspirin according to the decision of treating physician

Outcomes
There was some discrepancy between the outcomes listed in the protocol and reported in the study Methods and Results.

Primary outcomes: vascular death or major thrombosis (non-fatal major arterial and venous thrombotic events, i.e. MI, stroke, pulmonary embolism, DVT, TIA) (not listed in the protocol, reported). Vascular death or major thrombosis or major hemorrhage (fatal, intracranial, retroperitoneal, requiring blood transfusion or surgery) (listed in the protocol and reported)

Secondary outcomes: all-cause mortality (listed in the protocol and reported); total thrombotic events (major thrombosis and superficial thrombophlebitis [vein thrombosis]) (listed in the protocol, reported); minor thrombotic events (superficial thrombophlebitis [vein thrombosis]) (listed in the protocol and reported); major thrombotic events (MI, stroke, TIA, PE, DVT) (listed in the protocol and not reported as separate outcome); fatal and non-fatal cerebrovascular and cardiac events (not listed in the protocol, listed as outcome in Methods section, not reported); events contributing to primary outcomes 
WAPS 2005 (Continued)

separately (listed in the protocol and reported); fatal and non-fatal major hemorrhage (listed and reported); minor hemorrhage (listed and reported); all hemorrhages (not listed in the protocol, reported); any adverse event leading to treatment withdrawal

For all outcomes clear definitions provided and all verified by objective diagnostic methods

\begin{tabular}{ll}
\hline Funding source & NR \\
\hline Notes & $\begin{array}{l}\text { Originally planned sample size was } 500 \text { participants per arm. Following interim analysis for safety after } \\
3 \text { years the trial was stopped for futility, as recruitment was poorer than expected and transmission of } \\
\text { data from centres was delayed }\end{array}$ \\
& Additional information on study design and results obtained from Dr Finazzi
\end{tabular}

\section{Risk of bias}

\begin{tabular}{|c|c|c|}
\hline Bias & Authors' judgement & Support for judgement \\
\hline $\begin{array}{l}\text { Random sequence genera- } \\
\text { tion (selection bias) }\end{array}$ & Low risk & A program based on the biased-coin algorithm \\
\hline $\begin{array}{l}\text { Allocation concealment } \\
\text { (selection bias) }\end{array}$ & Low risk & Central randomization \\
\hline $\begin{array}{l}\text { Blinding of participants } \\
\text { and personnel (perfor- } \\
\text { mance bias) } \\
\text { Obj. }\end{array}$ & Low risk & $\begin{array}{l}\text { Objective or objectively verified outcomes: thrombotic events and bleeding: } \\
\text { no blinding, due to objective diagnostic verification or definition lack of blind- } \\
\text { ing will likely not influence the outcome }\end{array}$ \\
\hline $\begin{array}{l}\text { Blinding of outcome as- } \\
\text { sessment (detection bias) } \\
\text { Obj. }\end{array}$ & Low risk & $\begin{array}{l}\text { All outcomes: blinded endpoint adjudication by external committee blinded to } \\
\text { participants' treatment assignment. Each event was validated independently } \\
\text { by two evaluators, and disagreement between the evaluators was assessed by } \\
\text { the chairman of the study }\end{array}$ \\
\hline
\end{tabular}

Incomplete outcome data Unclear risk (attrition bias)

All outcomes
Discontinuations, withdrawals and missing data:

intervention: 5/54 discontinued, but followed and included in the analyses

control: 4/55 discontinued, but followed and included in the analyses

The authors reported following the ITT principle; however, according to the information from the author 6 participants were not followed; not clear from which group, the number of participants in the analysis equals the number of participants randomized, not clear how the participants not followed were included in the analysis

\begin{tabular}{lll}
\hline $\begin{array}{l}\text { Selective reporting (re- } \\
\text { porting bias) }\end{array}$ & High risk & $\begin{array}{l}\text { Protocol available; discrepancies between the outcomes listed in the protocol } \\
\text { and in the study, all important outcomes reported }\end{array}$ \\
\hline Other bias & High risk & $\begin{array}{l}\text { Originally planned sample size was } 500 \text { patients per arm. Following interim } \\
\text { analysis for safety after } 3 \text { years the trial was stopped for futility, as recruitment } \\
\text { was poorer than expected and transmission of data from centres was delayed. }\end{array}$
\end{tabular}

\section{Study characteristics}

Methods Study type: parallel RCT

Antiplatelet and anticoagulant agents for secondary prevention of stroke and other thromboembolic events in people with antiphospholipid syndrome (Review)

Copyright $\odot 2020$ The Cochrane Collaboration. Published by John Wiley \& Sons, Ltd. 
Yamazaki 2009 (Continued)

Location: Japan

Number of centres: 1

Time frame of the study: NR

Follow-up: 3 years

Funding: Ministry of Health, Japan

Inclusion criteria: people with APS
Exclusion criteria: NR
Total number of participants: 60 parts
in aspirin + cilostazol group; 20 in asp
Characteristics
Age: NR
Sex: NR
Systemic lupus erythematosus: NR
Previous events: NR
Cardiovascular risk factors: NR
Antibodies present: NR

\begin{tabular}{ll}
\hline Interventions & Treatment groups \\
& - $100 \mathrm{mg} / \mathrm{d}$ aspirin alone group \\
- & $100 \mathrm{mg} / \mathrm{d}$ aspirin + cilostazol $100 \mathrm{mg}$ twice daily \\
- & $100 \mathrm{mg} / \mathrm{d}$ aspirin + warfarin (INR 2.0 to 2.5) group
\end{tabular}

Descriptions of treatments and concomitant treatment: NR

\begin{tabular}{ll}
\hline Outcomes & $\begin{array}{l}\text { Primary outcomes: recurrence of stroke based on brain MRI } \\
\text { Secondary outcomes: NR }\end{array}$ \\
\hline Funding source & NR \\
\hline Notes & $\begin{array}{l}\text { All } 3 \text { groups were planned to be followed up for } 3 \text { years; however; group treated with } 100 \mathrm{mg} / \mathrm{d} \text { aspirin } \\
\text { alone was discontinued after a year for "humanitarian" reasons; several attempts to contact the au- } \\
\text { thors for additional information were unsuccessful. }\end{array}$
\end{tabular}

\section{Risk of bias}

\begin{tabular}{lll}
\hline Bias & Authors' judgement & Support for judgement \\
\hline $\begin{array}{l}\text { Random sequence genera- } \\
\text { tion (selection bias) }\end{array}$ & Unclear risk & No details apart from "patients were randomly treated" \\
\hline $\begin{array}{l}\text { Allocation concealment } \\
\text { (selection bias) }\end{array}$ & Unclear risk & Insufficient information provided \\
\hline $\begin{array}{l}\text { Blinding of participants } \\
\text { and personnel (perfor- } \\
\text { mance bias) }\end{array}$ & Unclear risk & Insufficient information provided \\
\end{tabular}

Antiplatelet and anticoagulant agents for secondary prevention of stroke and other thromboembolic events in people with 
Yamazaki 2009 (Continued)

Obj.

\begin{tabular}{|c|c|c|}
\hline $\begin{array}{l}\text { Blinding of outcome as- } \\
\text { sessment (detection bias) } \\
\text { Obj. }\end{array}$ & Unclear risk & Insufficient information provided \\
\hline $\begin{array}{l}\text { Incomplete outcome data } \\
\text { (attrition bias) } \\
\text { All outcomes }\end{array}$ & Unclear risk & Insufficient information to judge if all outcome data reported \\
\hline $\begin{array}{l}\text { Selective reporting (re- } \\
\text { porting bias) }\end{array}$ & Unclear risk & Insufficient information provided \\
\hline Other bias & High risk & $\begin{array}{l}\text { All } 3 \text { groups were planned to be followed up for } 3 \text { years; however; group treat- } \\
\text { ed with } 100 \mathrm{mg} / \mathrm{d} \text { aspirin alone was discontinued after a year for "humanitari- } \\
\text { an" reasons }\end{array}$ \\
\hline
\end{tabular}

AC: anticoagulant; AF: atrial fibrillation; AP: antiplatelet; APS: antiphospholipid syndrome; BMI: body mass index; CI: confidence interval;CT: computed tomography; DVT: deep vein thrombosis; INR: international normalized ratio; ITT: intention-to-treat; LA: lupus anticoagulant; MI: myocardial infarction; MRI: magnetic resonance imaging; NR: not reported; PE: pulmonary embolism; PP: per protocol; RCT: randomized controlled trial; SAE: serious adverse events; SD: standard deviation; TIA: transient ischemic attack; VKA: vitamin K antagonists; VTE: venous thromboembolism.

Characteristics of excluded studies [ordered by study ID]

\begin{tabular}{ll}
\hline Study & Reason for exclusion \\
\hline Cuadrado 2009 & $\begin{array}{l}\text { Irrelevant for the review population - mixed population - all with antibodies, most patients not ful- } \\
\text { filling APS criteria (66\%), 34\% meeting obstetric APS criteria, no separate results for those } 2 \text { groups }\end{array}$ \\
\hline
\end{tabular}

APS: antiphospholipid syndrome

Characteristics of studies awaiting classification [ordered by study ID]

\section{JASPRES}

Methods
Study type: open-label, parallel RCT, phase 4

Location: Japan

Number of centres: NR

Time frame of the study: 2006 to 2009

Follow-up: 2 years

Participants
Inclusion criteria: age $\geq 20$ years; APS diagnosed according to Sapporo criteria; cerebral infarct in history

Exclusion criteria: pregnant women or planned pregnancy; patients with contradictions to warfarin or antiplatelet drugs; patients required to take warfarin; severe hepatic, renal or cardiac failure

Total number of participants: planned sample size 100 participants

\section{Treatment groups}

- warfarin aimed at INR 2.0 for 2 years 
Descriptions of treatments and concomitant treatment: NR

\begin{tabular}{ll}
\hline Outcomes & Primary outcome: Cerebral Infarct Score in MRI \\
& Secondary outcomes: NR \\
\hline Notes & Funding: Health and Labour Sciences Research Grants \\
C000000342 \\
Information on the UMIN website that the study was terminated/discontinued. No more informa- \\
tion reported
\end{tabular}
bodies in people 1 month after stroke and that participants were randomly assigned to 2 groups, possible that it is an RCT

Location: Japan

\section{Number of centres: 1}

Time frame of the study: NR

Follow-up: NR

Participants Inclusion criteria: antiphospholipid antibodies; history of ischemic stroke

Exclusion criteria: NR

Total number of participants: 250 participants

Characteristics: NR

Interventions

Treatment groups

- single antiplatelet therapy

- combination of antiplatelet and anticoagulation therapy

\section{Descriptions of treatments and concomitant treatment: NR}

\begin{tabular}{ll}
\hline Outcomes & Primary outcome: recurrence of stroke \\
& Secondary outcomes: incidences of: anti-s2-glycoprotein I (anti-s2-GPI) antibodies, IgG anticardi- \\
olipin (IgG aCL), lupus anticoagulant, phosphatidylserine dependent anti-prothrombin antibody \\
(PS-PT), antiphosphatidylserine antibody (PS), and antiphosphatidyl inositol antibody (PI)
\end{tabular}

Notes

Funding: NR

Trying to contact the study authors regarding details of the study methods and results

Methods Study type: RCT


Yamazaki 2007 (Continued)

\section{Location: Japan}

Number of centres: 1

Time frame of the study: NR

Follow-up: NR

\begin{tabular}{ll}
\hline Inclusion criteria: APS; cerebral infarction in history \\
Exclusion criteria: NR \\
Total number of participants: 30 participants randomized, 30 analyzed: 10 in low-dose aspirin \\
alone group; 10 in aspirin + cilostazol group; 10 in aspirin + warfarin group \\
Characteristics: NR \\
\hline Treatment groups \\
- low dose aspirin alone group \\
- aspirin + cilostazol group \\
Descriptions of treatments and concomitant treatment: concomitant treatment not reported \\
Primary outcome: change in plasma derived microparticle levels \\
Secondary outcomes: worsening of lacunar infarctions in MRI \\
\hline Futcomes & Funding: NR \\
& Possible that it is the same study as Yamazaki 2009 , but waiting for confirmation from the study au-
\end{tabular}

APS: antiphospholipid syndrome; MRI: magnetic resonance imaging; NR: not reported; RCT: randomized controlled trial; SD: standard deviation; TIA: transient ischemic attack; VTE: venous thromboembolism.

Characteristics of ongoing studies [ordered by study ID]

\section{ASTRO-APS}

Study name

Apixaban for the secondary prevention of thrombosis among patients with antiphospholipid syndrome: study rationale and design (ASTRO-APS)

Methods

Study type: phase 4 prospective, randomized, open-label blinded event pilot study

Location: USA

Number of centres: NR

Time frame of the study: February 2015 to December 2019

Follow-up: 13 months

\section{Participants}

Antiplatelet and anticoagulant agents for secondary prevention of stroke and other thromboembolic events in people with antiphospholipid syndrome (Review)

Copyright (c) 2020 The Cochrane Collaboration. Published by John Wiley \& Sons, Ltd.

Inclusion criteria: age $\geq 18$ years; a clinical diagnosis of the APS and receiving anticoagulation (warfarin with a target INR of 2.5, 3.0 or 3.5 or another anticoagulant and willing to be randomized to study interventions); at least 6 months of anticoagulation for the indication of thrombosis completed, no acute neurologic symptoms associated with thrombosis, CVA, or TIA for a minimum of 6 months; consent to contact the participant's anticoagulation provider for the information on INRs, dosing and any adverse events; negative pregnancy test within 24 hours prior to the start of study drug; no breastfeeding; women of childbearing potential - contraception for the duration of treat- 
ment; males who are sexually active with women of childbearing potential must agree to follow instructions for method(s) of contraception for treatment and a total of 93 days post-treatment completion (azoospermic males and women of childbearing potential who are continuously not heterosexually active are exempt from contraceptive requirements. Pregnancy test still needed); agreement to undergo brain MRI

Exclusion criteria: another indication for long-term anticoagulation not approved by FDA for apixaban; a life expectancy of less than 1 year; not able to attend follow-up appointments; participating in another trial within the last 30 days or in a conflicting clinical trial; concomitant dual antiplatelet therapy; taking aspirin of dose $>165 \mathrm{mg} / \mathrm{d}$; hemoglobin $<8 \mathrm{mg} / \mathrm{dL}$; PLT $<50,000 / \mathrm{mL}$; serum creatinine level of $>2.5 \mathrm{mg} / \mathrm{dL}(221 \mu \mathrm{mol} / \mathrm{L})$ or $\mathrm{CrCl}<25 \mathrm{~mL} / \mathrm{min}$; ALT or AST $>2$ times the upper limit of the normal range; total bilirubin more than $1.5 \times \mathrm{ULN}$; active cancer and treatment for it within the last 3 months; receiving a CYP3A4 inhibitor and P-gp inhibitor; receiving a CYP3A4 and P-gp inducer; intend to become pregnant or breast feed within the next year; allergy to apixaban, rivaroxaban, or edoxaban; history of thrombosis while receiving warfarin at a target INR of 2 to 3 and assigned a higher target INR by the treating clinician; active pathological bleeding; a history of arterial thromboembolism; requires clopidogrel, ticagrelor, prasugrel, or another P2Y12 inhibitor; a history of catastrophic APS (CAPS); radiographic evidence of prior arterial thrombosis on MRI

Total number of participants: estimated 200 participants

Interventions

\section{Treatment groups}

- apixaban $2.5 \mathrm{mg}$, twice daily

- warfarin at a target INR of $\geq 2.0$ as prescribed before study

Descriptions of treatments and concomitant treatment: concomitant treatment not reported

Primary outcomes: rate of thrombosis (arterial and/or venous) and vascular death, major bleeding and clinically relevant non-major bleeding

Secondary outcomes: rate of the net clinical benefit outcome of thrombosis and bleeding, patient accrual regarding definite APS criteria; patient satisfaction using Anti-Clot Treatment Scale

\begin{tabular}{ll}
\hline Starting date & February 2015 \\
\hline Contact information & Scott Woller \\
& Intermountain Medical Center \\
& University of Utah School of Medicine, Eccles Outpatient Care Center \\
& 5169S Cottonwood St Suite \#307, \\
& Murray, UT 84107, USA. \\
& Email: scott.woller@imail.org \\
\hline Notes & Funding: grant paid to the Intermountain Medical Center, Murray UT, by Bristol-Meyers-Squibb \\
& NCT02295475 \\
\hline
\end{tabular}

\section{RISAPS}

Study name $\quad$ Rlvaroxaban for Stroke Patients With AntiPhospholipid Syndrome (RISAPS)

\begin{tabular}{ll}
\hline Methods & Study type: randomized, controlled, phase II/III, non-inferiority trial \\
Location: NR
\end{tabular}

\section{Number of centres: NR}


RISAPS (Continued)

\section{Time frame of the study: NR}

Follow-up: 24 months

Participants

Inclusion criteria: patients must be confirmed as having persistent antiphospholipid antibodies; One or more of: a) ischemic stroke; b) TIA (one or more episodes) with evidence of ischemic injury on brain MRI and diagnosed by a clinician with expertise in stroke; c) brain infarcts (territorial or subcortical) or white matter hyperintensities (WMH) of presumed vascular origin on brain MRI, with or without cognitive impairment; and an expert clinical opinion that anticoagulation is a reasonable treatment options; Women must be on adequate contraception, barrier or hormonal, unless postmenopausal or sterilized

Exclusion criteria: pregnant or lactating women; severe renal impairment creatinine clearance (Cockcroft \& Gault: Appendix 2) $<30 \mathrm{~mL} / \mathrm{min}$ (i.e. $29 \mathrm{~mL} / \mathrm{min}$ or less); liver function tests ALT $>2 \mathrm{x}$ ULN;

Cirrhotic patients with Child Pugh B or C; thrombocytopenia (platelets $<75 \times 109 / \mathrm{L}$ ); non-adherence on warfarin (based on clinical assessment); patients on azole antifungals (e.g. ketoconazole, itraconazole); patients on HIV protease inhibitors (e.g. ritonavir); patients on strong CYP3A4 inducers (e.g. rifampicin, phenytoin, carbamazepine, phenobarbital or St John's Wort); patients on dronedarone; patients less than 18 years of age; refusal to consent to the site informing GP and healthcare professional responsible for anticoagulation care of participation; contraindications to MRI (e.g. cardiac pacemaker, severe claustrophobia, inability to lie flat: patients who do not meet local safety rules for MRI); patients at high risk of bleeding and not suitable for anticoagulation therapy; previous known allergy or intolerance to warfarin or rivaroxaban; women planning to become pregnant within the 2-year follow-up period; patients with a known galactose intolerance, total lactase deficiency or galactose malabsorption

Total number of patients: 140 participants randomized 1:1

\section{Treatment groups}

- rivaroxaban $15 \mathrm{mg}$ twice daily orally for 24 months

- warfarin (as per standard care) to maintain a target INR of 3.5 (range 3.0-4.0)

Descriptions of treatments and concomitant treatment: concomitant treatment not reported

Outcomes

Primary outcomes: the rate of change in brain white matter hyperintensity (WMH) volume on MRI, a surrogate marker of ischemic damage, assessed on the 3D FLAIR sequence, between baseline and 24 months follow up

Secondary outcomes: health economics analysis, clinical measures of efficacy- vascular events, clinical measures of efficacy - death, a composite outcome measure of thrombotic events, including arterial, venous microvascular and death, safety events - bleeding all bleeds, safety - all serious adverse events, safety- cerebral micro bleeds, anti-coagulation intensity- rivaroxaban anti-Xa levels, anti-coagulation intensity-warfarin, changes in total brain volume, white matter volume and grey matter volume on T1w volumetric images on MRI, composite of clinical cardiac and cerebrovascular outcomes using MACCE

\begin{tabular}{ll}
\hline Starting date & Estimated study start date: January 2019 \\
\hline Contact information & RISAPS Trial Manager: risaps@ucl.ac.uk \\
\hline Notes & Funding: NR \\
& NCT03684564 \\
\hline
\end{tabular}

ALT: alanine aminotransferase; AST: aspartate aminotransferase; APS: antiphospholipid syndrome; BP: blood pressure; CIN: cervical intra-epithelial neoplasia; CrCl: creatine clearance; CVA: cerebrovascular accident; FDA: Food and Drug Administration; GFR: glomerular filtration rate;GI: gastrointestinal; HBV/HCV: hepatitis B/C virus; GPT: glutamic-pyruvic acid transaminase;INR: international normalized ratio;LMWH: low-molecular-weight heparin;MRI: magnetic resonance imaging;NR: not reported; NSAIDS: non-steroidal anti-inflammatory

Antiplatelet and anticoagulant agents for secondary prevention of stroke and other thromboembolic events in people with 55 antiphospholipid syndrome (Review)

Copyright (C) 2020 The Cochrane Collaboration. Published by John Wiley \& Sons, Ltd. 
drugs; PLT: platelet; RCT: randomized controlled trial; TIA: transient ischemic attack; ULN: upper limit of normal; VKA: vitamin K antagonists.

\section{DATA AND ANALYSES}

Comparison 1. NOAC (rivaroxaban) versus standard dose VKA

\begin{tabular}{|c|c|c|c|c|}
\hline Outcome or subgroup title & No. of studies & $\begin{array}{l}\text { No. of partici- } \\
\text { pants }\end{array}$ & Statistical method & Effect size \\
\hline $\begin{array}{l}\text { 1.1 Any thromboembolic event at the } \\
\text { longest follow-up }\end{array}$ & 3 & 425 & $\begin{array}{l}\text { Risk Ratio (M-H, Random, } \\
95 \% \mathrm{Cl})\end{array}$ & $4.08[0.48,34.79]$ \\
\hline $\begin{array}{l}\text { 1.2 Major bleeding at the longest fol- } \\
\text { low-up }\end{array}$ & 3 & 425 & $\begin{array}{l}\text { Risk Ratio (M-H, Random, } \\
95 \% \mathrm{Cl})\end{array}$ & $1.10[0.45,2.68]$ \\
\hline $\begin{array}{l}1.3 \text { Major bleeding at the longest fol- } \\
\text { low-up - calculated from log hazard } \\
\text { ratio }\end{array}$ & 2 & & $\begin{array}{l}\text { Hazard Ratio (IV, Random, } \\
95 \% \mathrm{CI} \text { ) }\end{array}$ & $1.15[0.46,2.87]$ \\
\hline $\begin{array}{l}\text { 1.4 All-cause mortality at the longest } \\
\text { follow-up }\end{array}$ & 3 & 425 & $\begin{array}{l}\text { Risk Ratio (M-H, Random, } \\
95 \% \mathrm{Cl})\end{array}$ & $1.45[0.44,4.78]$ \\
\hline 1.5 Stroke at the longest follow-up & 3 & 425 & $\begin{array}{l}\text { Risk Ratio (M-H, Random, } \\
95 \% \mathrm{Cl})\end{array}$ & $\begin{array}{l}14.13[1.87 \\
106.81]\end{array}$ \\
\hline 1.6 TIA at the longest follow-up & 3 & & $\begin{array}{l}\text { Risk Ratio (M-H, Random, } \\
95 \% \mathrm{Cl})\end{array}$ & Subtotals only \\
\hline 1.7 VTE at the longest follow-up & 3 & 425 & $\begin{array}{l}\text { Risk Ratio (M-H, Random, } \\
95 \% \mathrm{Cl})\end{array}$ & $0.96[0.20,4.49]$ \\
\hline $\begin{array}{l}\text { 1.8 Myocardial infarction at the } \\
\text { longest follow-up }\end{array}$ & 3 & 425 & $\begin{array}{l}\text { Risk Ratio (M-H, Random, } \\
95 \% \mathrm{Cl})\end{array}$ & $7.23[0.38,137.08]$ \\
\hline $\begin{array}{l}1.9 \text { Other thrombotic events at the } \\
\text { longest follow-up }\end{array}$ & 3 & & $\begin{array}{l}\text { Risk Ratio (M-H, Random, } \\
95 \% \mathrm{Cl})\end{array}$ & Subtotals only \\
\hline 1.9.1 microvascular thrombosis & 1 & 115 & $\begin{array}{l}\text { Risk Ratio (M-H, Random, } \\
95 \% \mathrm{Cl})\end{array}$ & Not estimable \\
\hline $\begin{array}{l}\text { 1.9.2 arterial events other than stroke } \\
\text { and } \mathrm{MI}\end{array}$ & 2 & 310 & $\begin{array}{l}\text { Risk Ratio (M-H, Random, } \\
95 \% \mathrm{Cl} \text { ) }\end{array}$ & $0.67[0.11,3.90]$ \\
\hline 1.10 Mean Quality of life at day 180 & 1 & & $\begin{array}{l}\text { Mean Difference (IV, Random, } \\
95 \% \mathrm{CI} \text { ) }\end{array}$ & $\begin{array}{l}\text { Totals not select- } \\
\text { ed }\end{array}$ \\
\hline 1.10.1 Health utility & 1 & & $\begin{array}{l}\text { Mean Difference (IV, Random, } \\
95 \% \mathrm{CI} \text { ) }\end{array}$ & $\begin{array}{l}\text { Totals not select- } \\
\text { ed }\end{array}$ \\
\hline 1.10.2 Health state & 1 & & $\begin{array}{l}\text { Mean Difference (IV, Random, } \\
95 \% \mathrm{CI} \text { ) }\end{array}$ & $\begin{array}{l}\text { Totals not select- } \\
\text { ed }\end{array}$ \\
\hline $\begin{array}{l}1.11 \text { Clinically relevant non-major } \\
\text { bleeding at the longest follow up }\end{array}$ & 2 & 302 & $\begin{array}{l}\text { Risk Ratio (M-H, Random, } \\
95 \% \mathrm{Cl})\end{array}$ & $1.70[0.69,4.19]$ \\
\hline
\end{tabular}




\begin{tabular}{lllll}
\hline Outcome or subgroup title & No. of studies & $\begin{array}{l}\text { No. of partici- } \\
\text { pants }\end{array}$ & Statistical method & Effect size \\
\hline $\begin{array}{l}\text { 1.12 Minor bleeding at the longest fol- } \\
\text { low-up }\end{array}$ & 2 & 302 & $\begin{array}{l}\text { Risk Ratio (M-H, Random, } \\
95 \% \mathrm{Cl})\end{array}$ & $1.17[0.69,1.96]$ \\
\hline
\end{tabular}

\section{Analysis 1.1. Comparison 1: NOAC (rivaroxaban) versus standard dose VKA, Outcome 1: Any thromboembolic event at the longest follow-up}

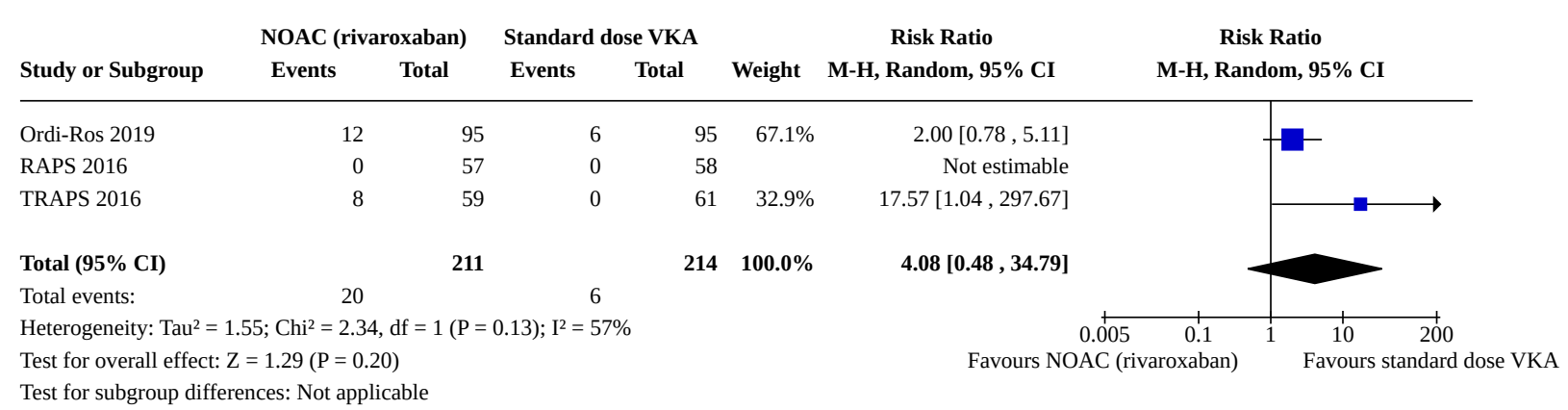

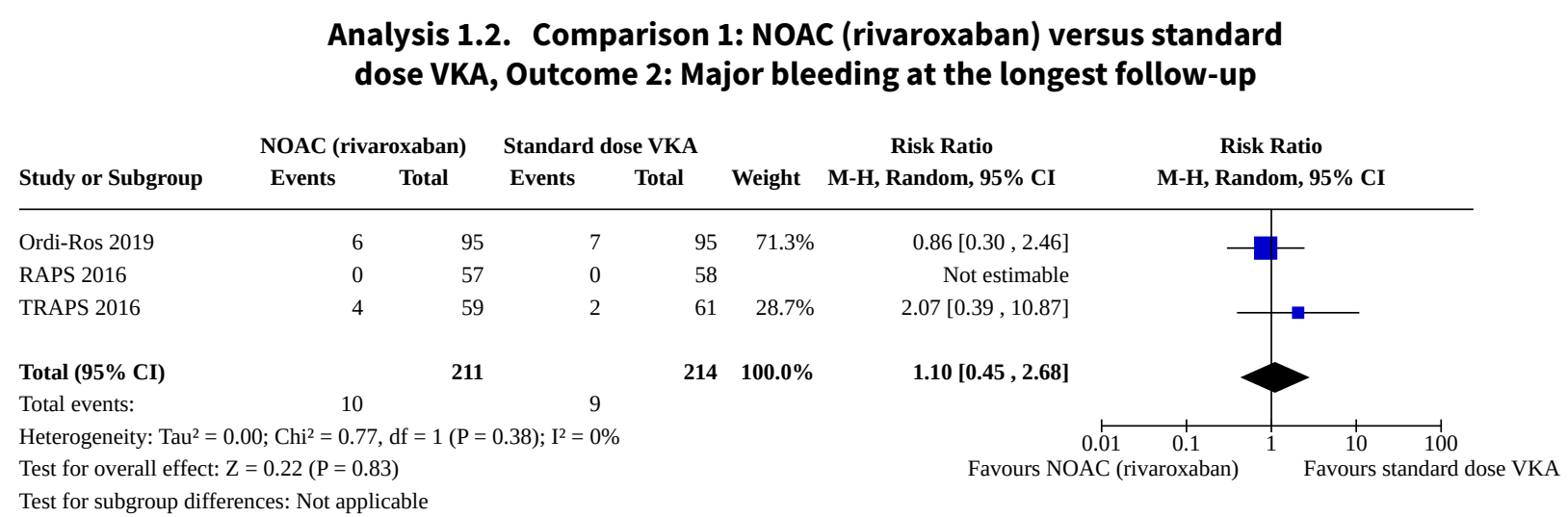

Analysis 1.3. Comparison 1: NOAC (rivaroxaban) versus standard dose VKA, Outcome 3: Major bleeding at the longest follow-up - calculated from log hazard ratio

$\begin{array}{lccccc} & & & \text { Hazard Ratio } & \text { Hazard Ratio } \\ \text { Study or Subgroup } & \log [\text { Hazard Ratio] } & \text { SE } & \text { Weight } & \text { IV, Random, 95\% CI } & \text { IV, Random, 95\% CI }\end{array}$

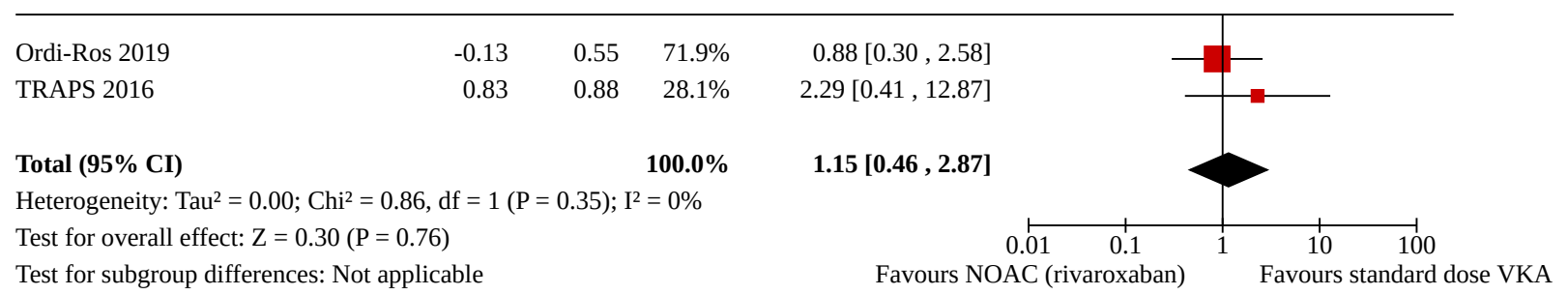




\section{Analysis 1.4. Comparison 1: NOAC (rivaroxaban) versus standard dose VKA, Outcome 4: All-cause mortality at the longest follow-up}

\begin{tabular}{|c|c|c|c|c|c|c|c|}
\hline \multirow[b]{2}{*}{ Study or Subgroup } & \multicolumn{2}{|c|}{ NOAC (rivaroxaban) } & \multicolumn{2}{|c|}{ Standard dose VKA } & \multirow[b]{2}{*}{ Weight } & \multirow{2}{*}{$\begin{array}{c}\text { Risk Ratio } \\
\text { M-H, Random, 95\% CI }\end{array}$} & \multirow{2}{*}{$\begin{array}{c}\text { Risk Ratio } \\
\text { M-H, Random, 95\% CI }\end{array}$} \\
\hline & Events & Total & Events & Total & & & \\
\hline Ordi-Ros 2019 & 5 & 95 & 3 & 95 & $72.0 \%$ & $1.67[0.41,6.78]$ & \\
\hline RAPS 2016 & 0 & 57 & 1 & 58 & $14.0 \%$ & $0.34[0.01,8.15]$ & - \\
\hline TRAPS 2016 & 1 & 59 & 0 & 61 & $14.0 \%$ & $3.10[0.13,74.61]$ & \\
\hline Total $(95 \% \mathrm{CI})$ & & 211 & & 214 & $100.0 \%$ & $1.45[0.44,4.78]$ & \\
\hline Total events: & 6 & & 4 & & & & \\
\hline \multicolumn{4}{|c|}{ Heterogeneity: Tau $^{2}=0.00 ; \mathrm{Chi}^{2}=1.06, \mathrm{df}=2(\mathrm{P}=0.59) ; \mathrm{I}^{2}=0 \%$} & & & 0.01 & 0.1 \\
\hline Test for overall effect & $=0.62(\mathrm{P}=0$. & & & & & Favours NOAC ( & varoxaban) \\
\hline
\end{tabular}

Test for overall effect: $\mathrm{Z}=0.62(\mathrm{P}=0.54)$

Test for subgroup differences: Not applicable

Analysis 1.5. Comparison 1: NOAC (rivaroxaban) versus standard dose VKA, Outcome 5: Stroke at the longest follow-up

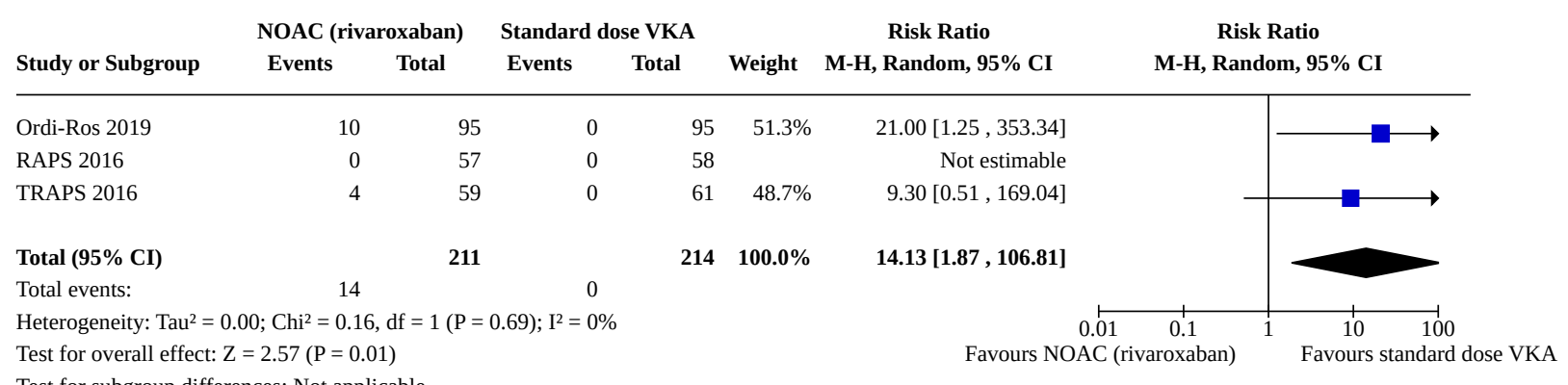

Analysis 1.6. Comparison 1: NOAC (rivaroxaban) versus standard dose VKA, Outcome 6: TIA at the longest follow-up

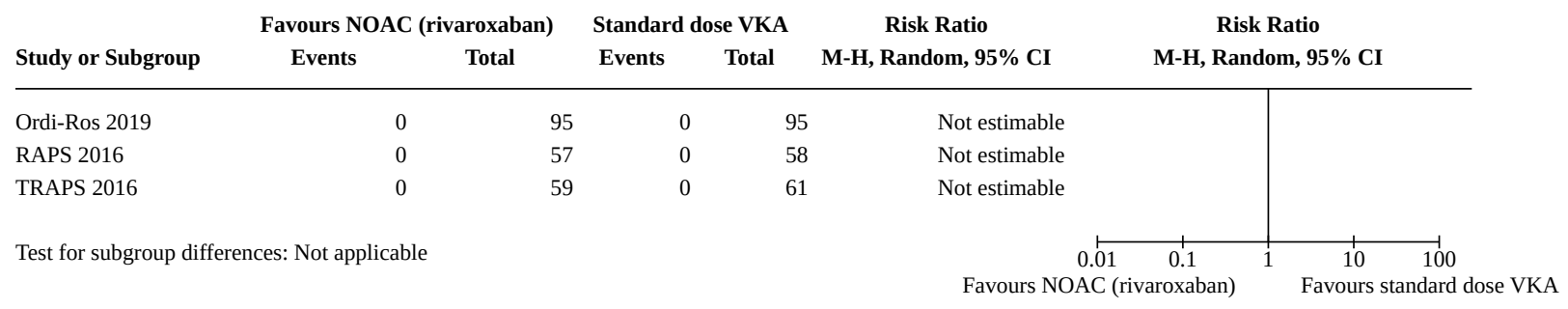

Analysis 1.7. Comparison 1: NOAC (rivaroxaban) versus standard dose VKA, Outcome 7: VTE at the longest follow-up

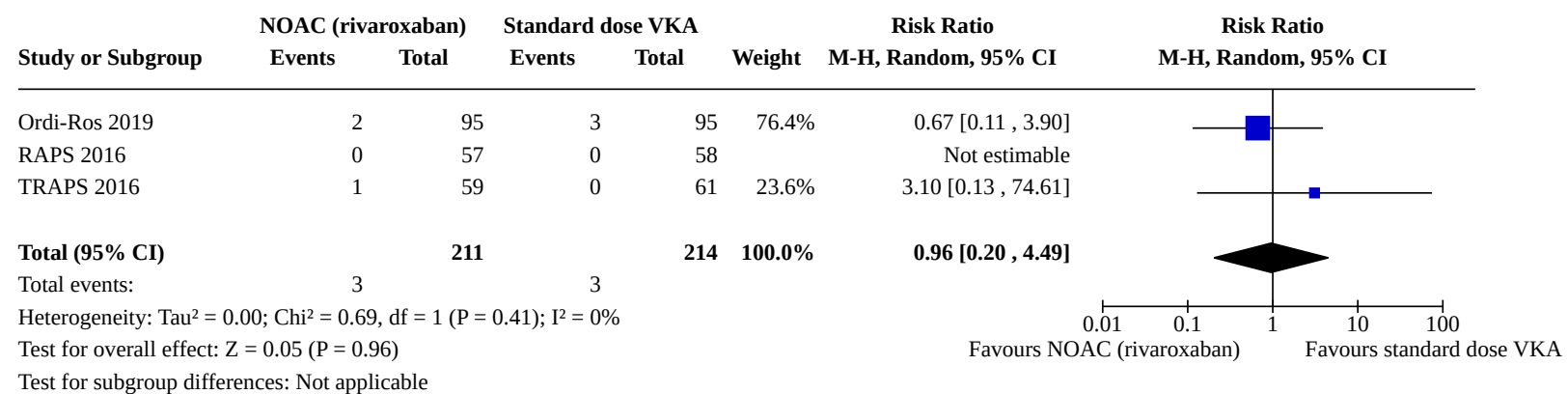


Analysis 1.8. Comparison 1: NOAC (rivaroxaban) versus standard dose VKA, Outcome 8: Myocardial infarction at the longest follow-up

\begin{tabular}{|c|c|c|c|c|c|c|c|c|c|}
\hline \multirow{3}{*}{$\begin{array}{l}\text { Study or Subgroup } \\
\text { Ordi-Ros } 2019\end{array}$} & \multicolumn{2}{|c|}{ NOAC (rivaroxaban) } & \multicolumn{2}{|c|}{ Standard dose VKA } & \multirow[b]{2}{*}{ Weight } & \multirow{2}{*}{$\begin{array}{c}\text { Risk Ratio } \\
\text { M-H, Random, 95\% CI }\end{array}$} & \multirow{2}{*}{\multicolumn{2}{|c|}{$\begin{array}{c}\text { Risk Ratio } \\
\text { M-H, Random, 95\% CI }\end{array}$}} & \\
\hline & Events & Total & Events & Total & & & & & \\
\hline & 0 & 95 & 0 & 95 & & Not estimable & & & \\
\hline RAPS 2016 & 0 & 57 & 0 & 58 & & Not estimable & & & \\
\hline TRAPS 2016 & 3 & 59 & 0 & 61 & $100.0 \%$ & $7.23[0.38,137.08]$ & & & $\longrightarrow$ \\
\hline Total (95\% CI) & & 211 & & 214 & $100.0 \%$ & $7.23[0.38,137.08]$ & & & \\
\hline Total events: & 3 & & 0 & & & & & & \\
\hline Heterogeneity: Not a & able & & & & & 0.01 & 0.1 & 10 & 100 \\
\hline Test for overall effect & $=1.32(\mathrm{P}=0$ & & & & & Favours NOAC ( & varoxaban) & Favours st & andard dose VKA \\
\hline
\end{tabular}

Analysis 1.9. Comparison 1: NOAC (rivaroxaban) versus standard dose VKA, Outcome 9: Other thrombotic events at the longest follow-up

\begin{tabular}{cccccccc} 
& \multicolumn{2}{c}{ NOAC (rivaroxaban) } & \multicolumn{2}{c}{ Standard dose VKA } & \multicolumn{2}{c}{ Risk Ratio } & Risk Ratio \\
Study or Subgroup & Events & Total & Events & Total & Weight & M-H, Random, 95\% CI & M-H, Random, 95\% CI
\end{tabular}

1.9.1 microvascular thrombosis RAPS 2016 Subtotal (95\% CI)

$57 \quad 0$

58

Not estimable

Total events:

0

$57 \quad 0$

58

Not estimable

Heterogeneity: Not applicable

Test for overall effect: Not applicable

1.9.2 arterial events other than stroke and MI Ordi-Ros 2019

TRAPS 2016

Subtotal (95\% CI)

$0 \quad 59$

Total events: 2

154

Heterogeneity: Not applicable

Test for overall effect: $\mathrm{Z}=0.45(\mathrm{P}=0.65)$

$\begin{array}{rrrrr}95 & 3 & 95 & 100.0 \% & 0.67[0.11,3.90] \\ 59 & 0 & 61 & & \text { Not estimable } \\ \mathbf{1 5 4} & & \mathbf{1 5 6} & \mathbf{1 0 0 . 0 \%} & \mathbf{0 . 6 7}[\mathbf{0 . 1 1}, \mathbf{3 . 9 0}]\end{array}$

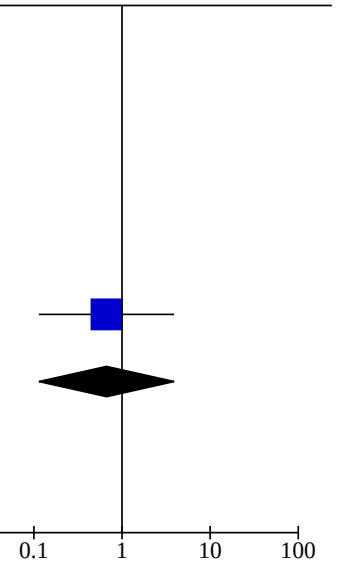

Analysis 1.10. Comparison 1: NOAC (rivaroxaban) versus standard dose VKA, Outcome 10: Mean Quality of life at day 180

\begin{tabular}{|c|c|c|c|c|c|c|c|c|}
\hline & NOA & riva & jan) & Sta & d do & KA & Mean Difference & Mean Difference \\
\hline Study or Subgroup & Mean & SD & Total & Mean & SD & Total & IV, Random, 95\% CI & IV, Random, 95\% CI \\
\hline
\end{tabular}

1.10.1 Health utility

RAPS 2016

$\begin{array}{rrrrrrr}0.82 & 0.15 & 56 & 0.78 & 0.15 & 55 & 0.04[-0.02,0.10] \\ 80 & 13.47 & 56 & 73 & 13.47 & 56 & 7.00[2.01,11.99]\end{array}$

1.10.2 Health state

RAPS 2016

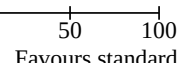

Favours NOAC (rivaroxaban) $\quad$ Favours standard dose VKA 
Analysis 1.11. Comparison 1: NOAC (rivaroxaban) versus standard dose VKA, Outcome 11: Clinically relevant non-major bleeding at the longest follow up

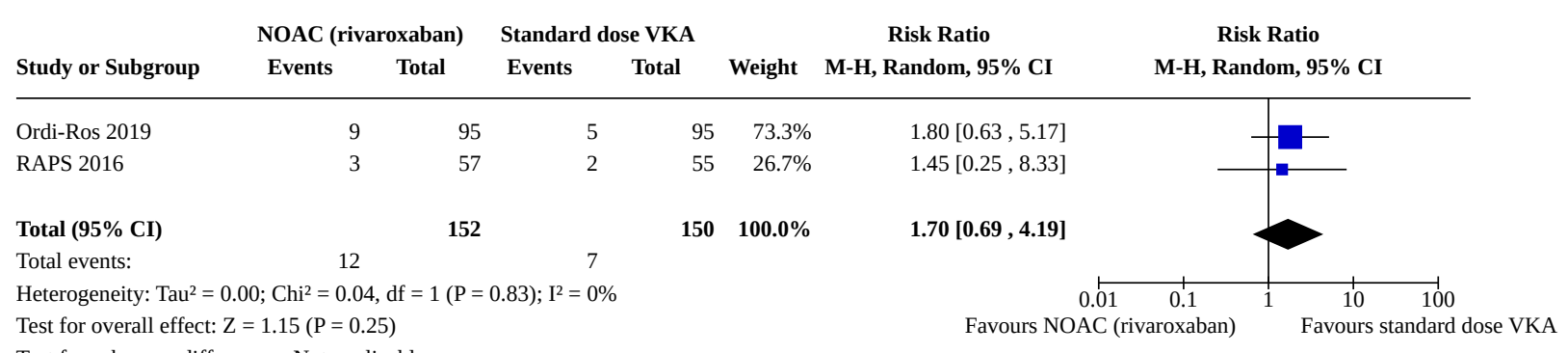

Test for subgroup differences: Not applicable

\begin{tabular}{|c|c|c|c|c|c|c|c|}
\hline \multirow[b]{2}{*}{ Study or Subgroup } & \multicolumn{2}{|c|}{ NOAC (rivaroxaban) } & \multicolumn{2}{|c|}{ Standard dose VKA } & \multirow[b]{2}{*}{ Weight } & \multirow{2}{*}{$\begin{array}{c}\text { Risk Ratio } \\
\text { M-H, Random, 95\% CI }\end{array}$} & \multirow{2}{*}{$\begin{array}{c}\text { Risk Ratio } \\
\text { M-H, Random, 95\% CI }\end{array}$} \\
\hline & Events & Total & Events & Total & & & \\
\hline Ordi-Ros 2019 & 16 & 95 & 14 & 95 & $62.6 \%$ & $1.14[0.59,2.21]$ & \\
\hline RAPS 2016 & 10 & 57 & 8 & 55 & $37.4 \%$ & $1.21[0.51,2.83]$ & \\
\hline Total (95\% CI) & & 152 & & 150 & $100.0 \%$ & $1.17[0.69,1.96]$ & \\
\hline Total events: & 26 & & 22 & & & & \\
\hline \multicolumn{6}{|c|}{ Heterogeneity: Tau $^{2}=0.00 ; \mathrm{Chi}^{2}=0.01, \mathrm{df}=1(\mathrm{P}=0.92) ; \mathrm{I}^{2}=0 \%$} & $\begin{array}{r}0.01 \\
\text { rs NOAC }\end{array}$ & $\begin{array}{cc}1 & 10 \\
\text { Favours s }\end{array}$ \\
\hline
\end{tabular}

\section{Comparison 2. High dose VKA versus standard dose VKA}

\begin{tabular}{|c|c|c|c|c|}
\hline Outcome or subgroup title & No. of studies & $\begin{array}{l}\text { No. of partici- } \\
\text { pants }\end{array}$ & Statistical method & Effect size \\
\hline $\begin{array}{l}\text { 2.1 Any thromboembolic event at the } \\
\text { longest follow-up }\end{array}$ & 2 & 223 & $\begin{array}{l}\text { Risk Ratio (M-H, Random, } \\
95 \% \mathrm{CI})\end{array}$ & $2.22[0.79,6.23]$ \\
\hline $\begin{array}{l}2.2 \text { Any thromboembolic event at the } \\
\text { longest follow-up - calculated from log } \\
\text { hazard ratio }\end{array}$ & 2 & & $\begin{array}{l}\text { Hazard Ratio (IV, Random, } \\
95 \% \mathrm{CI})\end{array}$ & $2.17[0.74,6.31]$ \\
\hline $\begin{array}{l}\text { 2.3 Major bleeding at the longest fol- } \\
\text { low-up }\end{array}$ & 2 & 223 & $\begin{array}{l}\text { Risk Ratio (M-H, Random, } \\
95 \% \mathrm{CI})\end{array}$ & $0.74[0.24,2.25]$ \\
\hline $\begin{array}{l}\text { 2.4 Major bleeding at the longest fol- } \\
\text { low-up - calculated from log hazard ratio }\end{array}$ & 2 & & $\begin{array}{l}\text { Hazard Ratio (IV, Random, } \\
95 \% \mathrm{CI})\end{array}$ & $0.83[0.25,2.72]$ \\
\hline $\begin{array}{l}2.5 \text { All-cause mortality at the longest fol- } \\
\text { low-up }\end{array}$ & 2 & 223 & $\begin{array}{l}\text { Risk Ratio (M-H, Random, } \\
95 \% \mathrm{Cl})\end{array}$ & $1.53[0.27,8.79]$ \\
\hline 2.6 Stroke at the longest follow-up & 2 & 223 & $\begin{array}{l}\text { Risk Ratio (M-H, Random, } \\
95 \% \mathrm{Cl})\end{array}$ & $1.37[0.26,7.12]$ \\
\hline 2.7 TIA at the longest follow-up & 2 & 223 & $\begin{array}{l}\text { Risk Ratio (M-H, Random, } \\
95 \% \mathrm{Cl} \text { ) }\end{array}$ & $2.04[0.19,21.81]$ \\
\hline
\end{tabular}




\begin{tabular}{|c|c|c|c|c|}
\hline Outcome or subgroup title & No. of studies & $\begin{array}{l}\text { No. of partici- } \\
\text { pants }\end{array}$ & Statistical method & Effect size \\
\hline 2.8 VTE at the longest follow-up & 2 & 223 & $\begin{array}{l}\text { Risk Ratio (M-H, Random, } \\
95 \% \mathrm{Cl})\end{array}$ & $4.44[0.77,25.72]$ \\
\hline $\begin{array}{l}2.9 \text { Myocardial infarction at the longest } \\
\text { follow-up }\end{array}$ & 2 & 223 & $\begin{array}{l}\text { Risk Ratio (M-H, Random, } \\
95 \% \mathrm{Cl})\end{array}$ & $1.04[0.07,16.16]$ \\
\hline $\begin{array}{l}2.10 \text { Other thrombotic events at the } \\
\text { longest follow-up (superficial vein throm- } \\
\text { bosis) }\end{array}$ & 1 & & $\begin{array}{l}\text { Risk Ratio (M-H, Random, } \\
95 \% \mathrm{Cl})\end{array}$ & Subtotals only \\
\hline $\begin{array}{l}\text { 2.11 Minor bleeding at the longest fol- } \\
\text { low-up }\end{array}$ & 1 & & $\begin{array}{l}\text { Risk Ratio (M-H, Random, } \\
95 \% \mathrm{Cl})\end{array}$ & Subtotals only \\
\hline $\begin{array}{l}\text { 2.12 Any bleeding at the longest fol- } \\
\text { low-up }\end{array}$ & 2 & 223 & $\begin{array}{l}\text { Risk Ratio (M-H, Random, } \\
95 \% \mathrm{Cl})\end{array}$ & $1.56[0.93,2.62]$ \\
\hline $\begin{array}{l}2.13 \text { Any bleeding at the longest fol- } \\
\text { low-up - calculated from log hazard ratio }\end{array}$ & 2 & & $\begin{array}{l}\text { Hazard Ratio (IV, Random, } \\
95 \% \mathrm{Cl} \text { ) }\end{array}$ & $2.03[1.12,3.68]$ \\
\hline
\end{tabular}

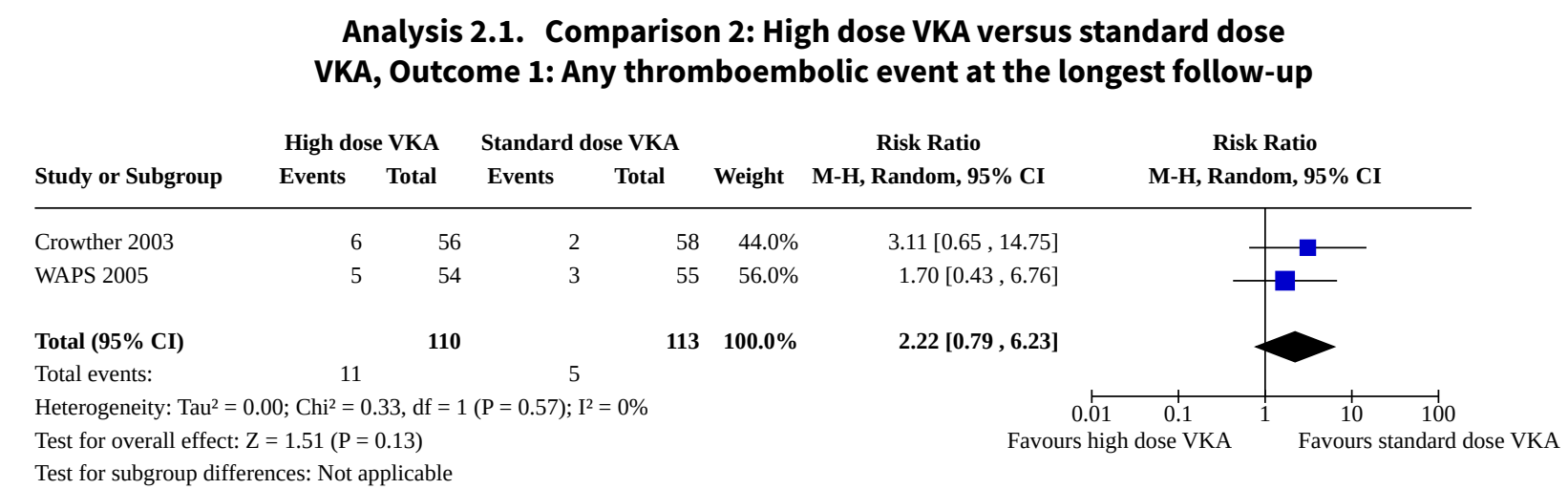

Analysis 2.2. Comparison 2: High dose VKA versus standard dose VKA, Outcome 2: Any thromboembolic event at the longest follow-up - calculated from log hazard ratio

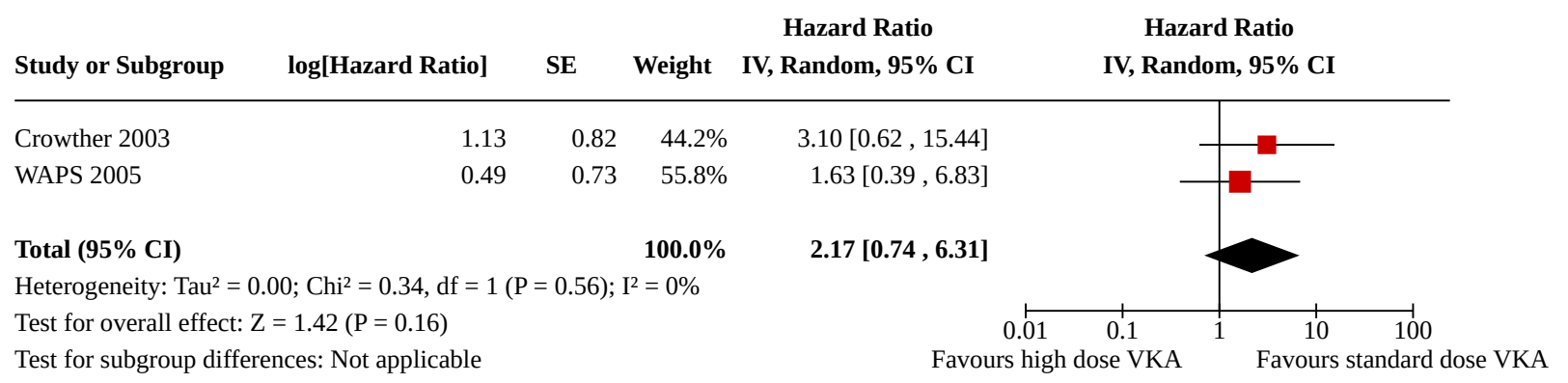


Analysis 2.3. Comparison 2: High dose VKA versus standard dose VKA, Outcome 3: Major bleeding at the longest follow-up

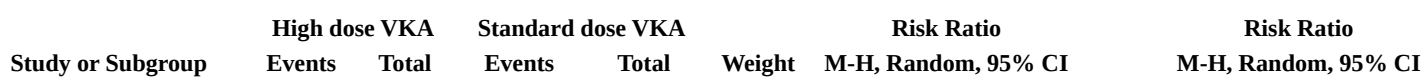

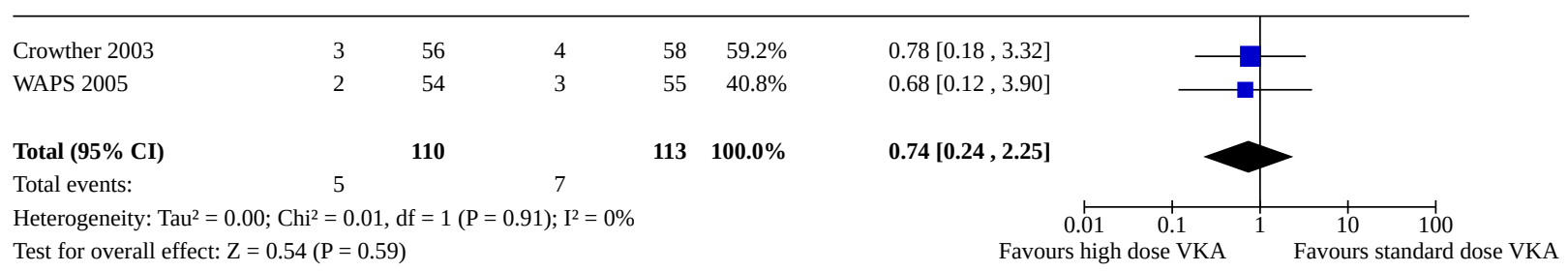

Favours high dose VKA Favours standard dose VKA

\section{Analysis 2.4. Comparison 2: High dose VKA versus standard dose VKA, Outcome} 4: Major bleeding at the longest follow-up - calculated from log hazard ratio

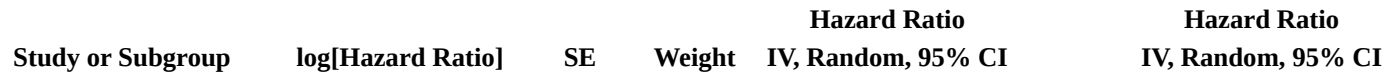

\begin{tabular}{lrrrr}
\hline Crowther 2003 & 0 & 0.81 & $55.8 \%$ & $1.00[0.20,4.89]$ \\
WAPS 2005 & -0.42 & 0.91 & $44.2 \%$ & $0.66[0.11,3.91]$ \\
\end{tabular}

Analysis 2.5. Comparison 2: High dose VKA versus standard dose VKA, Outcome 5: All-cause mortality at the longest follow-up

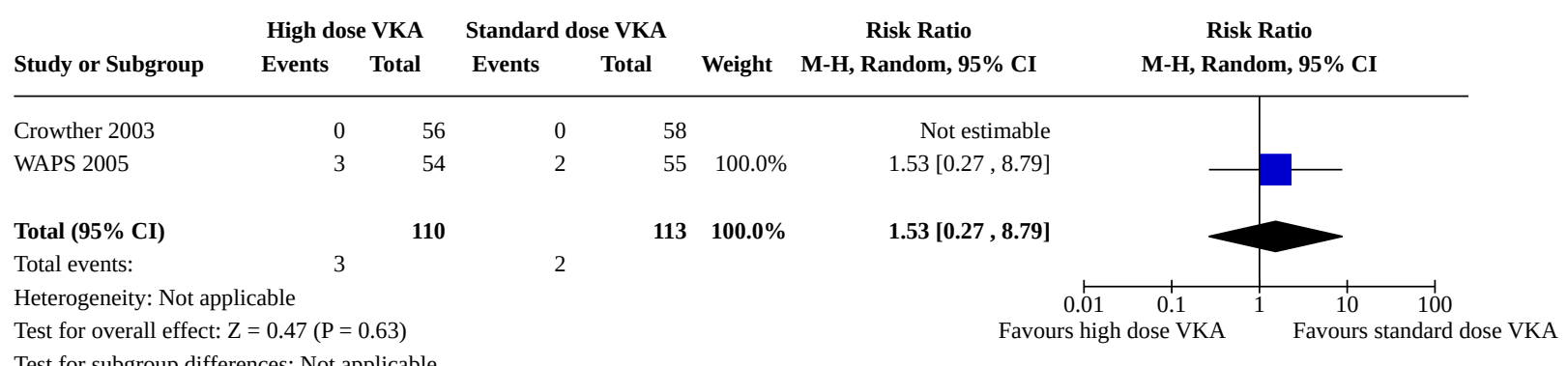

Analysis 2.6. Comparison 2: High dose VKA versus standard dose VKA, Outcome 6: Stroke at the longest follow-up

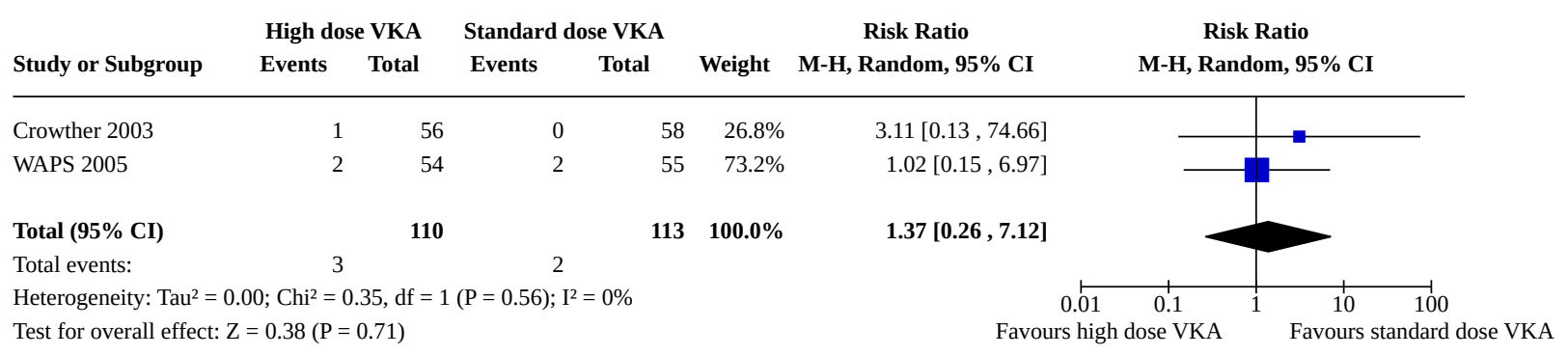


Analysis 2.7. Comparison 2: High dose VKA versus standard dose VKA, Outcome 7: TIA at the longest follow-up

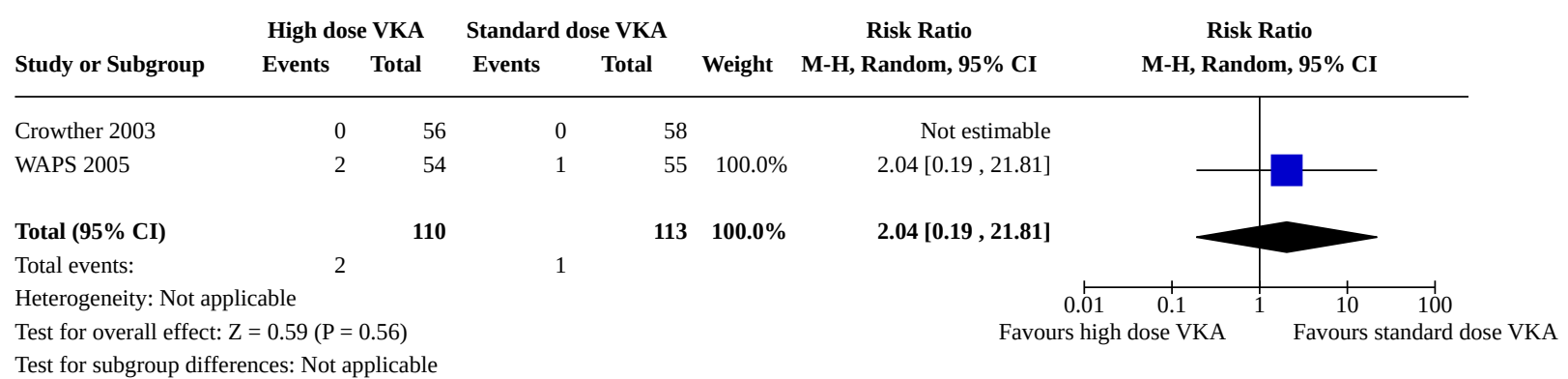

\section{Analysis 2.8. Comparison 2: High dose VKA versus standard dose VKA, Outcome 8: VTE at the longest follow-up}

\begin{tabular}{cccccccc} 
& \multicolumn{2}{c}{ High dose VKA } & \multicolumn{2}{c}{ Standard dose VKA } & \multicolumn{2}{c}{ Risk Ratio } & Risk Ratio \\
Study or Subgroup & Events & Total & Events & Total & Weight & M-H, Random, 95\% CI & M-H, Random, 95\% CI
\end{tabular}

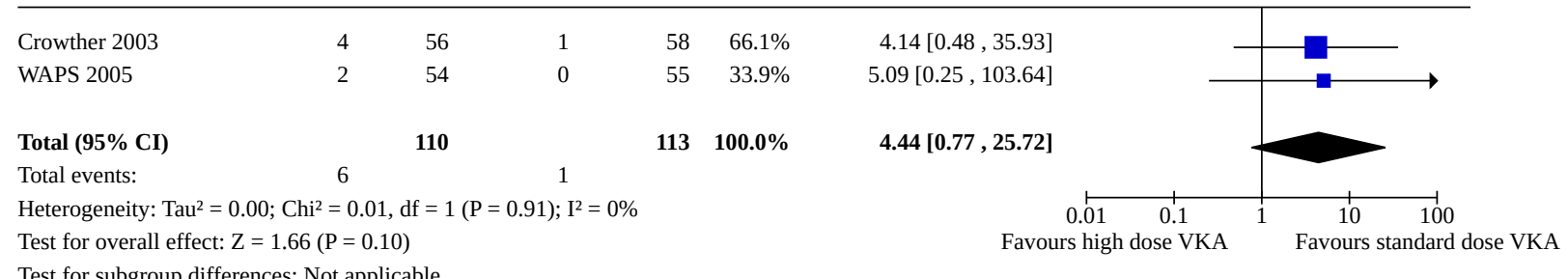

Test for subgroup differences: Not applicable

Analysis 2.9. Comparison 2: High dose VKA versus standard dose
VKA, Outcome 9: Myocardial infarction at the longest follow-up

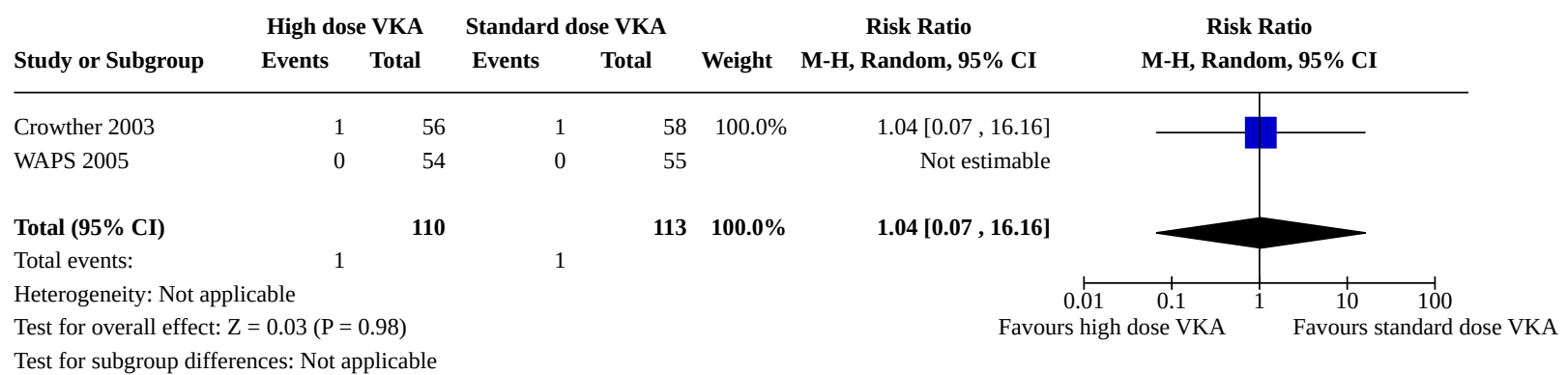

Analysis 2.10. Comparison 2: High dose VKA versus standard dose VKA, Outcome 10: Other thrombotic events at the longest follow-up (superficial vein thrombosis)

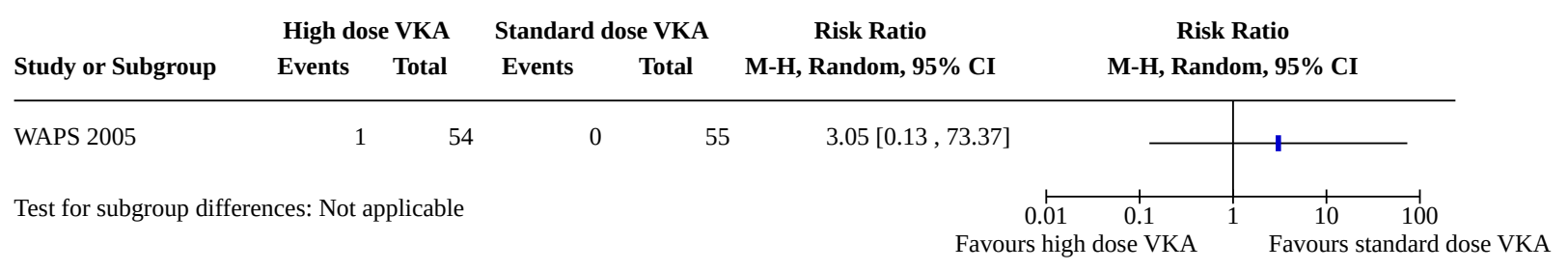


Analysis 2.11. Comparison 2: High dose VKA versus standard dose VKA, Outcome 11: Minor bleeding at the longest follow-up

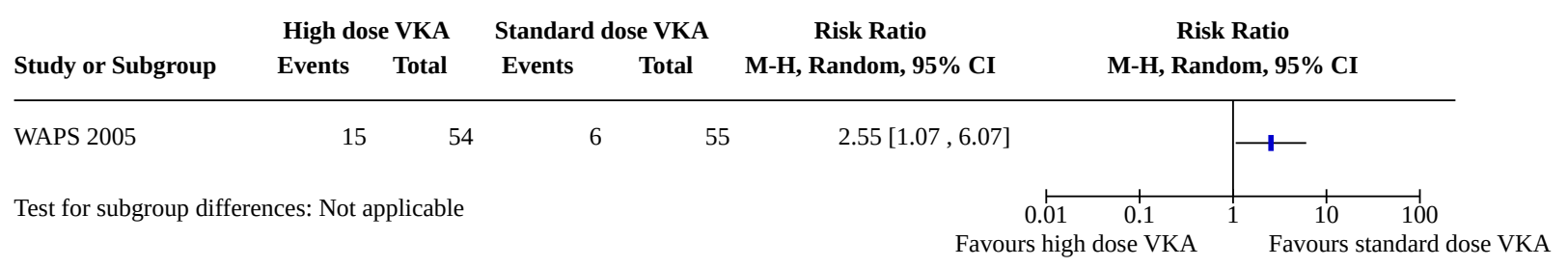

Analysis 2.12. Comparison 2: High dose VKA versus standard dose VKA, Outcome 12: Any bleeding at the longest follow-up

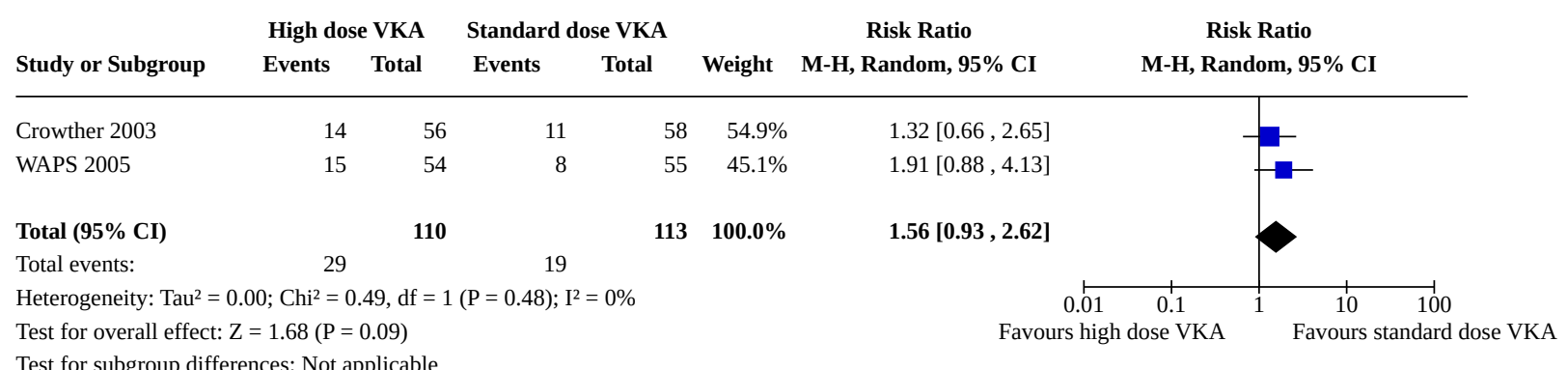

\section{Analysis 2.13. Comparison 2: High dose VKA versus standard dose VKA, Outcome 13: Any bleeding at the longest follow-up - calculated from log hazard ratio}

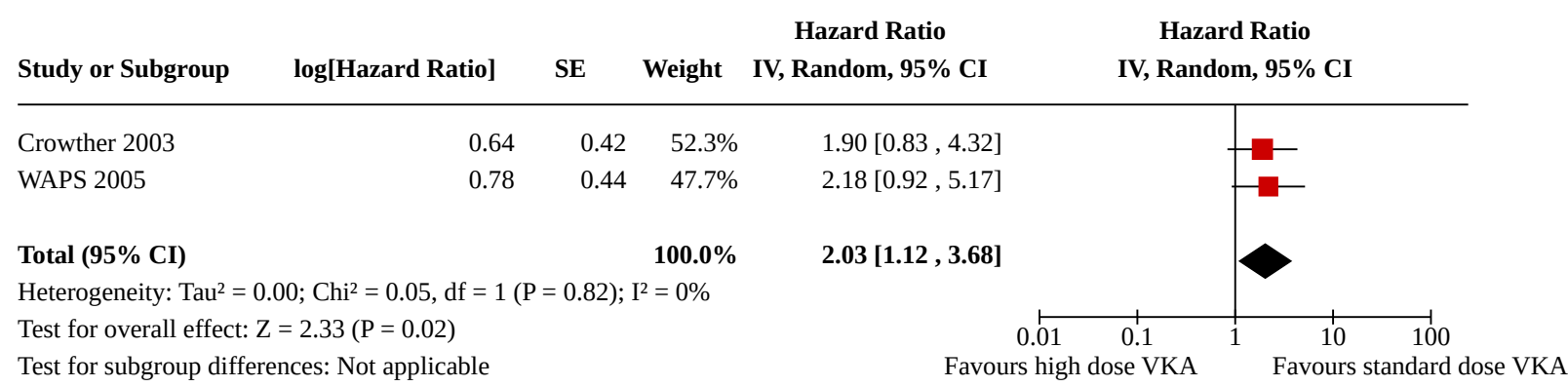

Comparison 3. Standard dose VKA plus single antiplatelet agent versus standard dose VKA

\begin{tabular}{lllll}
\hline Outcome or subgroup title & No. of studies & $\begin{array}{l}\text { No. of partici- } \\
\text { pants }\end{array}$ & Statistical method & Effect size \\
\hline $\begin{array}{l}\text { 3.1 Any thromboembolic event at the } \\
\text { longest follow up }\end{array}$ & 1 & & $\begin{array}{l}\text { Risk Ratio (M-H, Random, } \\
95 \% \mathrm{Cl})\end{array}$ & $\begin{array}{l}\text { Totals not select- } \\
\text { ed }\end{array}$ \\
\hline $\begin{array}{l}\text { 3.2 Major bleeding at the longest follow } \\
\text { up }\end{array}$ & 1 & $\begin{array}{l}\text { Risk Ratio (M-H, Random, } \\
95 \% \mathrm{Cl})\end{array}$ & $\begin{array}{l}\text { Totals not select- } \\
\text { ed }\end{array}$ \\
\hline $\begin{array}{l}\text { 3.3 All-cause mortality at the longest fol- } \\
\text { low up }\end{array}$ & 1 & Risk Ratio (M-H, Random, & Totals not select- \\
\hline
\end{tabular}




\begin{tabular}{|c|c|c|c|c|}
\hline Outcome or subgroup title & No. of studies & $\begin{array}{l}\text { No. of partici- } \\
\text { pants }\end{array}$ & Statistical method & Effect size \\
\hline 3.4 Stroke at the longest follow-up & 1 & & $\begin{array}{l}\text { Risk Ratio (M-H, Random, } \\
95 \% \mathrm{Cl})\end{array}$ & $\begin{array}{l}\text { Totals not select- } \\
\text { ed }\end{array}$ \\
\hline 3.5 TIA at the longest follow-up & 1 & & $\begin{array}{l}\text { Risk Ratio (M-H, Random, } \\
95 \% \mathrm{Cl})\end{array}$ & Subtotals only \\
\hline 3.6 VTE at the longest follow-up & 1 & & $\begin{array}{l}\text { Risk Ratio (M-H, Random, } \\
95 \% \mathrm{Cl} \text { ) }\end{array}$ & Subtotals only \\
\hline $\begin{array}{l}\text { 3.7 Myocardial infarction at the longest } \\
\text { follow-up }\end{array}$ & 1 & & $\begin{array}{l}\text { Risk Ratio (M-H, Random, } \\
95 \% \mathrm{Cl})\end{array}$ & Subtotals only \\
\hline $\begin{array}{l}\text { 3.8 Other thrombotic events at the } \\
\text { longest follow-up (hearing loss, retinal } \\
\text { artery thrombosis) }\end{array}$ & 1 & & $\begin{array}{l}\text { Risk Ratio (M-H, Random, } \\
95 \% \mathrm{Cl} \text { ) }\end{array}$ & $\begin{array}{l}\text { Totals not select- } \\
\text { ed }\end{array}$ \\
\hline $\begin{array}{l}3.9 \text { Minor bleeding at the longest fol- } \\
\text { low-up }\end{array}$ & 1 & & $\begin{array}{l}\text { Risk Ratio (M-H, Random, } \\
95 \% \mathrm{Cl})\end{array}$ & $\begin{array}{l}\text { Totals not select- } \\
\text { ed }\end{array}$ \\
\hline
\end{tabular}

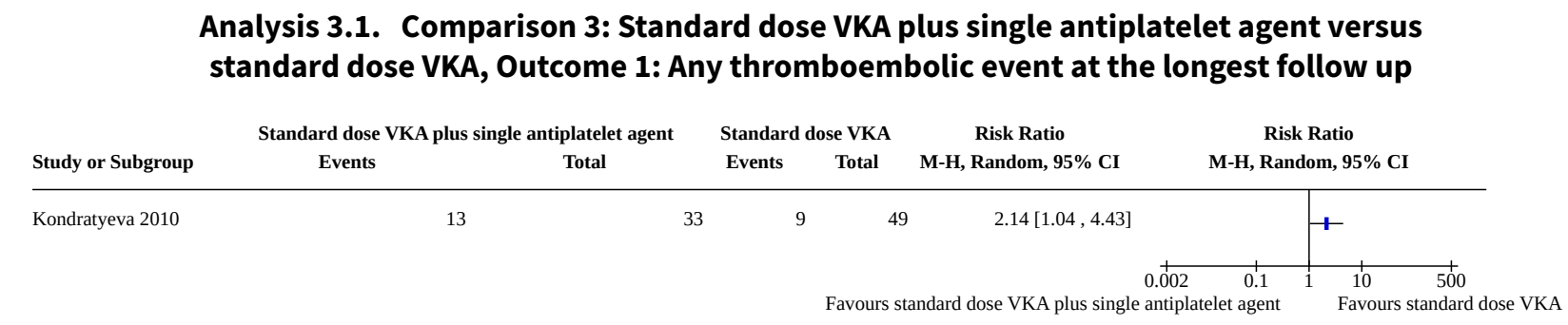

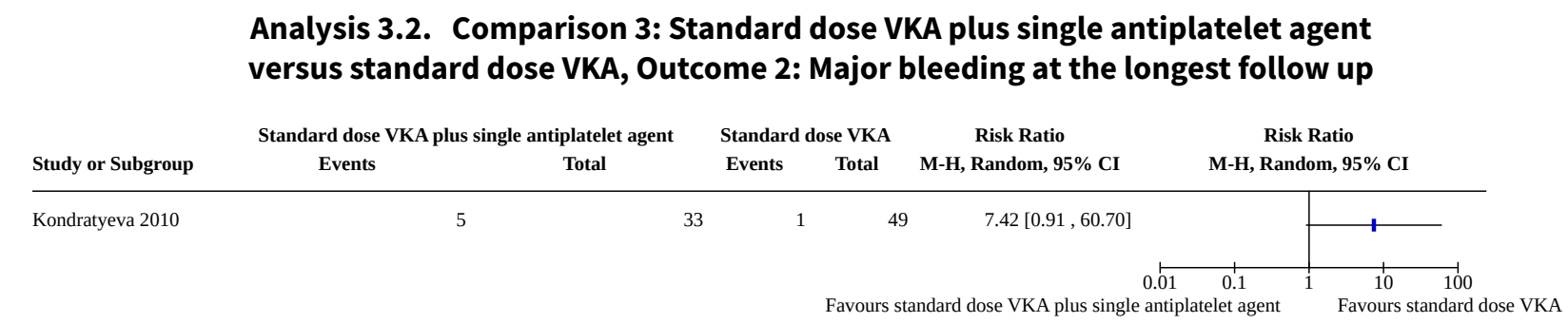

Analysis 3.3. Comparison 3: Standard dose VKA plus single antiplatelet agent versus standard dose VKA, Outcome 3: All-cause mortality at the longest follow up

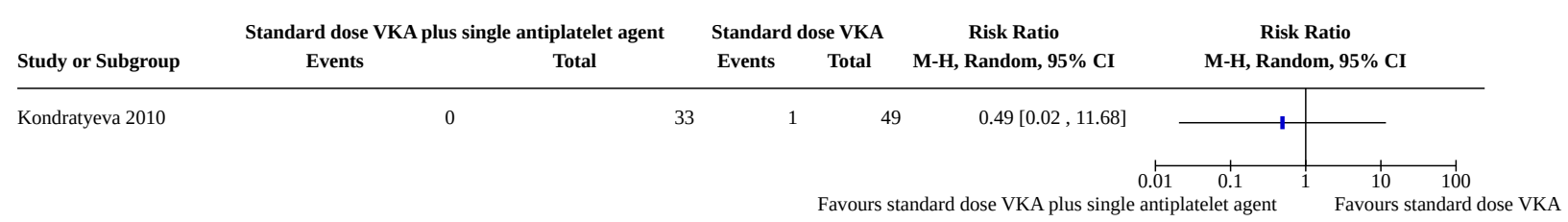


Analysis 3.4. Comparison 3: Standard dose VKA plus single antiplatelet agent versus standard dose VKA, Outcome 4: Stroke at the longest follow-up

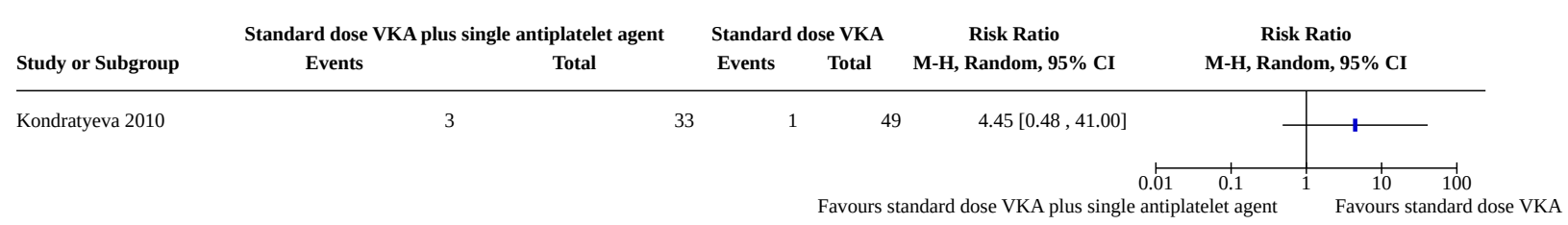

Analysis 3.5. Comparison 3: Standard dose VKA plus single antiplatelet agent versus standard dose VKA, Outcome 5: TIA at the longest follow-up

\begin{tabular}{|c|c|c|c|c|c|c|c|}
\hline \multirow[b]{2}{*}{ Study or Subgroup } & \multicolumn{2}{|c|}{ Standard dose VKA plus single antiplatelet agent } & \multicolumn{2}{|c|}{ Standard dose VKA } & \multirow{2}{*}{$\begin{array}{c}\text { Risk Ratio } \\
\text { M-H, Random, 95\% CI }\end{array}$} & \multirow{2}{*}{\multicolumn{2}{|c|}{$\begin{array}{c}\text { Risk Ratio } \\
\text { M-H, Random, 95\% CI }\end{array}$}} \\
\hline & Events & Total & Events & Total & & & \\
\hline Kondratyeva 2010 & 5 & 33 & 3 & 4 & $2.47[0.63,9.66]$ & & 1 \\
\hline
\end{tabular}

Analysis 3.6. Comparison 3: Standard dose VKA plus single antiplatelet agent versus standard dose VKA, Outcome 6: VTE at the longest follow-up

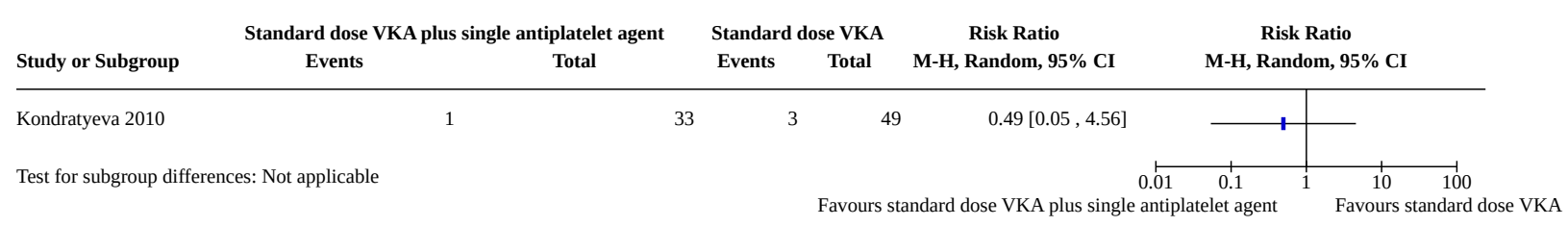

Analysis 3.7. Comparison 3: Standard dose VKA plus single antiplatelet agent versus standard dose VKA, Outcome 7: Myocardial infarction at the longest follow-up

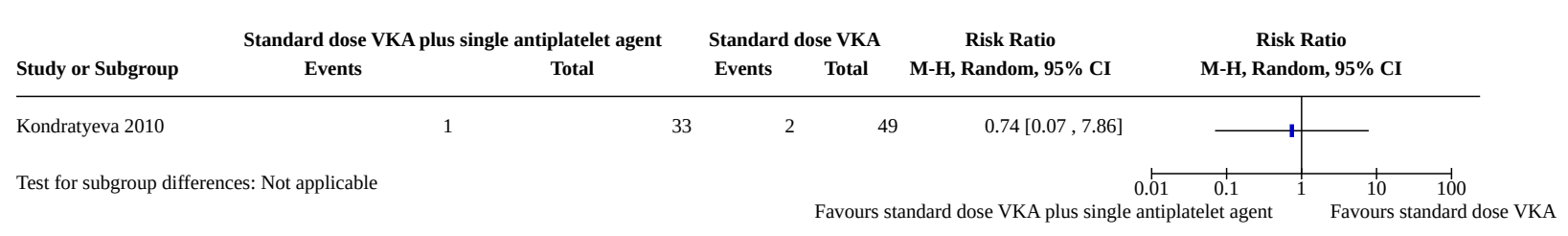

Analysis 3.8. Comparison 3: Standard dose VKA plus single antiplatelet agent versus standard dose VKA, Outcome 8: Other thrombotic events at the longest follow-up (hearing loss, retinal artery thrombosis)

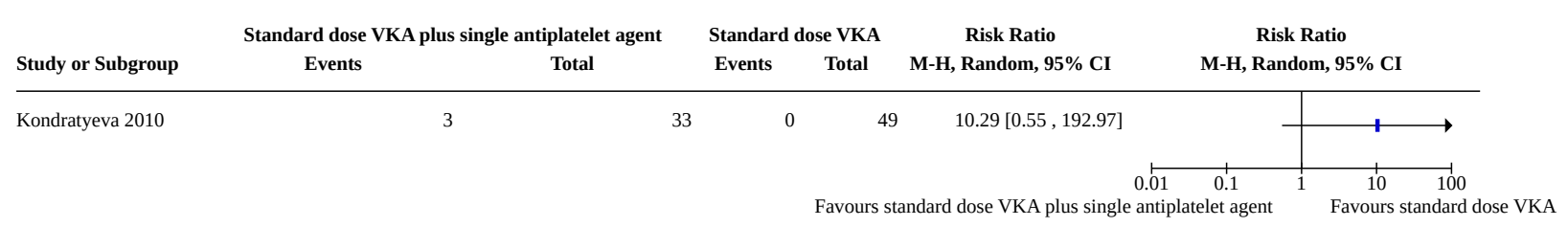


Analysis 3.9. Comparison 3: Standard dose VKA plus single antiplatelet agent versus standard dose VKA, Outcome 9: Minor bleeding at the longest follow-up

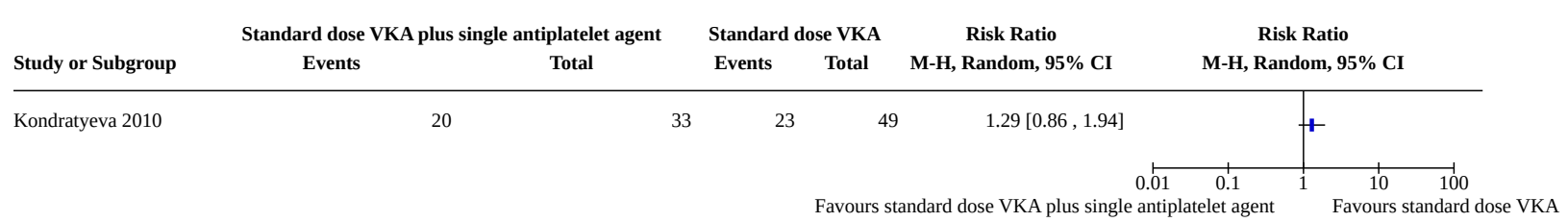

Comparison 4. Standard dose VKA plus single antiplatelet agent versus single antiplatelet agent

\begin{tabular}{|c|c|c|c|c|}
\hline Outcome or subgroup title & No. of studies & $\begin{array}{l}\text { No. of partici- } \\
\text { pants }\end{array}$ & Statistical method & Effect size \\
\hline $\begin{array}{l}4.1 \text { Major bleeding (minor cerebral haem- } \\
\text { orrhage) at a mean of } 3.9 \text { years }\end{array}$ & 1 & 20 & $\begin{array}{l}\text { Risk Ratio (M-H, Random, } \\
95 \% \mathrm{Cl})\end{array}$ & $0.40[0.02,8.78]$ \\
\hline 4.2 Stroke at 1-year follow-up & 1 & & $\begin{array}{l}\text { Risk Ratio (M-H, Random, } \\
95 \% \mathrm{Cl})\end{array}$ & $\begin{array}{l}\text { Totals not select- } \\
\text { ed }\end{array}$ \\
\hline $\begin{array}{l}4.3 \text { Minor bleeding (subcutaneous haem- } \\
\text { orrhage) at a mean of } 3.9 \text { years }\end{array}$ & 1 & 20 & $\begin{array}{l}\text { Risk Ratio (M-H, Random, } \\
95 \% \mathrm{Cl})\end{array}$ & $3.60[0.16,79.01]$ \\
\hline $\begin{array}{l}4.4 \mathrm{GI} \text { bleeding (no definition) at a mean } \\
\text { of } 3.9 \text { years }\end{array}$ & 1 & 20 & $\begin{array}{l}\text { Risk Ratio (M-H, Random, } \\
95 \% \mathrm{Cl})\end{array}$ & Not estimable \\
\hline
\end{tabular}

Analysis 4.1. Comparison 4: Standard dose VKA plus single antiplatelet agent versus single antiplatelet agent, Outcome 1: Major bleeding (minor cerebral haemorrhage) at a mean of 3.9 years

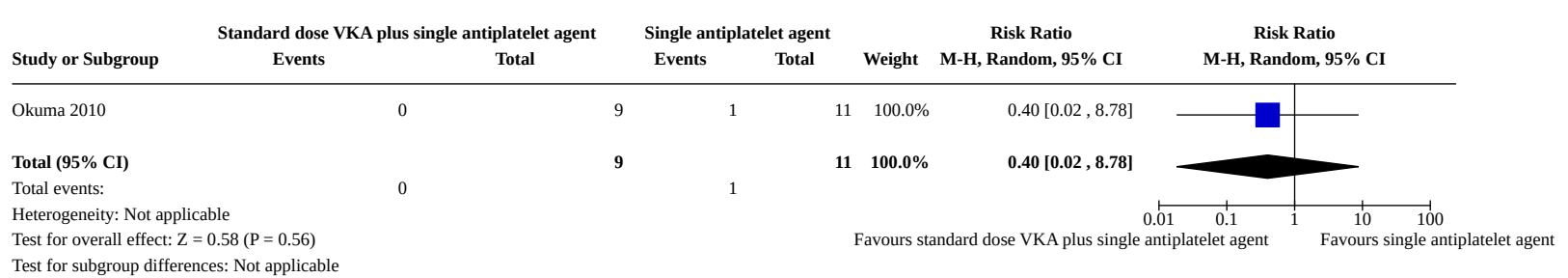

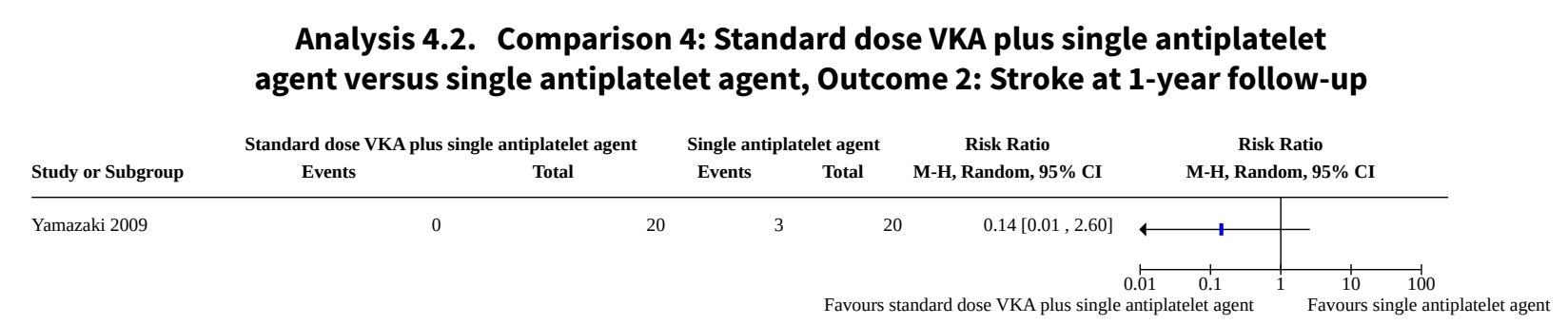

Antiplatelet and anticoagulant agents for secondary prevention of stroke and other thromboembolic events in people with antiphospholipid syndrome (Review)

Copyright (c) 2020 The Cochrane Collaboration. Published by John Wiley \& Sons, Ltd. 
Analysis 4.3. Comparison 4: Standard dose VKA plus single antiplatelet agent versus single antiplatelet agent, Outcome 3: Minor bleeding (subcutaneous haemorrhage) at a mean of 3.9 years

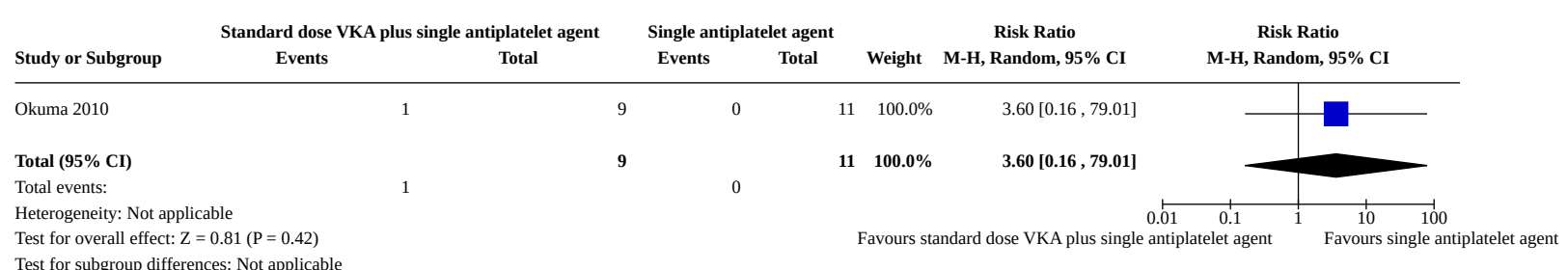

Analysis 4.4. Comparison 4: Standard dose VKA plus single antiplatelet agent versus single antiplatelet agent, Outcome 4: GI bleeding (no definition) at a mean of 3.9 years

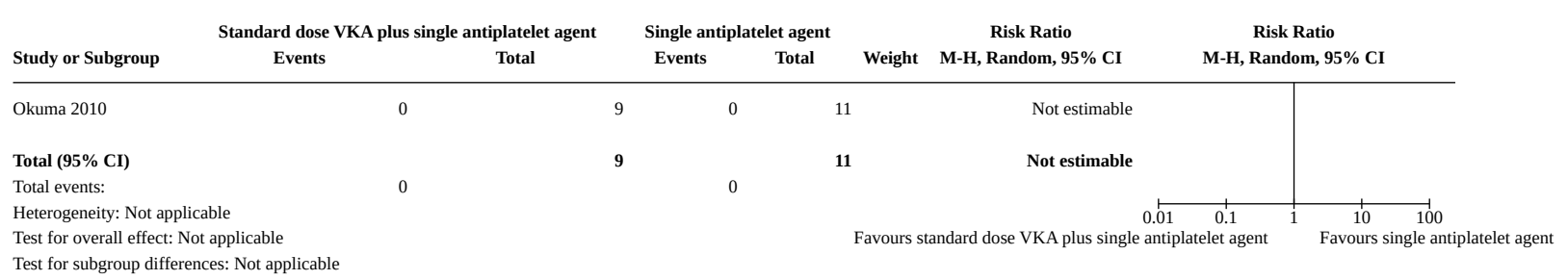

Comparison 5. Standard dose VKA plus antiplatelet agent vs dual antiplatelet therapy

\begin{tabular}{lllll}
\hline Outcome or subgroup title & No. of studies & $\begin{array}{l}\text { No. of partici- } \\
\text { pants }\end{array}$ & Statistical method & Effect size \\
\hline 5.1 Stroke at 3 years & 1 & & Risk Ratio (M-H, Random, 95\% Cl) & Totals not selected \\
\hline
\end{tabular}

Analysis 5.1. Comparison 5: Standard dose VKA plus antiplatelet agent vs dual antiplatelet therapy, Outcome 1: Stroke at 3 years

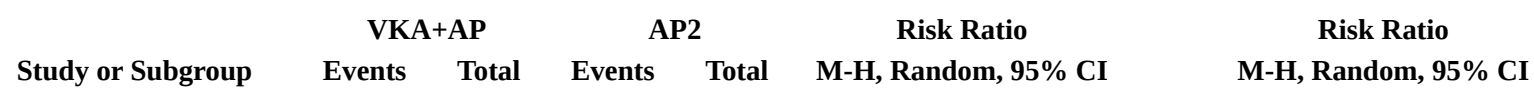

\begin{tabular}{|c|c|c|c|c|c|c|}
\hline \multirow[t]{2}{*}{ Yamazaki 2009} & 2 & 20 & 0 & 20 & $5.00[0.26,98.00]$ & 1 \\
\hline & & & & & $\begin{array}{cc}0.01 & 0.1 \\
\text { Favours } & \mathrm{VKA}+\mathrm{AC}\end{array}$ & $\begin{array}{c}10 \\
\text { Favours AP2 }\end{array}$ \\
\hline
\end{tabular}

Comparison 6. Dual antiplatelet therapy vs single antiplatelet agent

\begin{tabular}{lllll}
\hline Outcome or subgroup title & No. of studies & $\begin{array}{l}\text { No. of partici- } \\
\text { pants }\end{array}$ & Statistical method & Effect size \\
\hline 6.1 Stroke at 1 year & 1 & & Risk Ratio (M-H, Random, 95\% Cl) & Totals not selected \\
\hline
\end{tabular}




\section{Analysis 6.1. Comparison 6: Dual antiplatelet therapy vs single antiplatelet agent, Outcome 1: Stroke at 1 year}
AP2
AP1
Risk Ratio
Risk Ratio

Study or Subgroup

Events Tota

Events Total

M-H, Random, 95\% CI

M-H, Random, 95\% CI

Yamazaki 2009

$\begin{array}{llll}0 & 20 & 3 & 20\end{array}$

$0.14[0.01,2.60]$

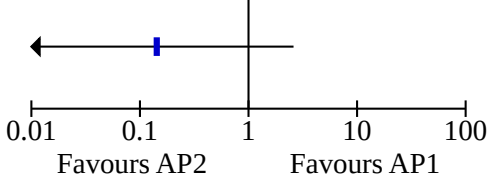

Comparison 7. Sensitivity analysis NOAC vs VKA

\begin{tabular}{|c|c|c|c|c|}
\hline Outcome or subgroup title & No. of studies & $\begin{array}{l}\text { No. of partici- } \\
\text { pants }\end{array}$ & Statistical method & Effect size \\
\hline $\begin{array}{l}7.1 \text { Best case any thromboembolic event at } \\
\text { the longest follow-up }\end{array}$ & 3 & 426 & $\begin{array}{l}\text { Risk Ratio (M-H, Random, } \\
95 \% \mathrm{Cl})\end{array}$ & $2.34[0.43,12.74]$ \\
\hline $\begin{array}{l}7.2 \text { Worst case any thromboembolic event } \\
\text { at the longest follow-up }\end{array}$ & 3 & 426 & $\begin{array}{l}\text { Risk Ratio (M-H, Random, } \\
95 \% \mathrm{Cl})\end{array}$ & $4.08[0.48,34.79]$ \\
\hline $\begin{array}{l}7.3 \text { Best case major bleeding at the longest } \\
\text { follow-up }\end{array}$ & 3 & 429 & $\begin{array}{l}\text { Risk Ratio (M-H, Random, } \\
95 \% \mathrm{Cl})\end{array}$ & $0.87[0.28,2.75]$ \\
\hline $\begin{array}{l}7.4 \text { Worst case major bleeding at the } \\
\text { longest follow-up }\end{array}$ & 3 & 429 & $\begin{array}{l}\text { Risk Ratio (M-H, Random, } \\
95 \% \mathrm{Cl})\end{array}$ & $1.10[0.45,2.68]$ \\
\hline $\begin{array}{l}7.5 \text { Best case all-cause death at the longest } \\
\text { follow-up }\end{array}$ & 3 & 426 & $\begin{array}{l}\text { Risk Ratio (M-H, Random, } \\
95 \% \mathrm{Cl})\end{array}$ & $1.32[0.40,4.29]$ \\
\hline $\begin{array}{l}7.6 \text { Worst case all-cause death at the } \\
\text { longest follow-up }\end{array}$ & 3 & 426 & $\begin{array}{l}\text { Risk Ratio (M-H, Random, } \\
95 \% \mathrm{Cl})\end{array}$ & $1.46[0.44,4.79]$ \\
\hline $\begin{array}{l}\text { 7.7 Best case clinically relevant non-major } \\
\text { bleeding at the longest follow up }\end{array}$ & 2 & 306 & $\begin{array}{l}\text { Risk Ratio (M-H, Random, } \\
95 \% \mathrm{Cl})\end{array}$ & $1.04[0.31,3.49]$ \\
\hline $\begin{array}{l}7.8 \text { Worst case clinically relevant non-ma- } \\
\text { jor bleeding at the longest follow up }\end{array}$ & 2 & 306 & $\begin{array}{l}\text { Risk Ratio (M-H, Random, } \\
95 \% \mathrm{Cl} \text { ) }\end{array}$ & $1.73[0.70,4.27]$ \\
\hline $\begin{array}{l}7.9 \text { Best case minor bleeding at the longest } \\
\text { follow-up }\end{array}$ & 2 & 306 & $\begin{array}{l}\text { Risk Ratio (M-H, Random, } \\
95 \% \mathrm{Cl})\end{array}$ & $1.01[0.62,1.66]$ \\
\hline $\begin{array}{l}\text { 7.10 Worst case minor bleeding at the } \\
\text { longest follow-up }\end{array}$ & 2 & 306 & $\begin{array}{l}\text { Risk Ratio (M-H, Random, } \\
95 \% \mathrm{Cl})\end{array}$ & $1.20[0.71,2.02]$ \\
\hline
\end{tabular}


Analysis 7.1. Comparison 7: Sensitivity analysis NOAC vs VKA, Outcome 1: Best case any thromboembolic event at the longest follow-up

\begin{tabular}{|c|c|c|c|c|c|c|c|}
\hline \multirow[b]{2}{*}{ Study or Subgroup } & \multicolumn{2}{|c|}{ NOAC (rivaroxaban) } & \multicolumn{2}{|c|}{ standard dose VKA } & \multirow[b]{2}{*}{ Weight } & \multirow{2}{*}{$\begin{array}{c}\text { Risk Ratio } \\
\text { M-H, Random, } 95 \% \text { CI }\end{array}$} & \multirow{2}{*}{$\begin{array}{c}\text { Risk Ratio } \\
\text { M-H, Random, 95\% CI }\end{array}$} \\
\hline & Events & Total & Events & Total & & & \\
\hline Ordi-Ros 2019 & 12 & 95 & 6 & 95 & $56.5 \%$ & $2.00[0.78,5.11]$ & \\
\hline RAPS 2016 & 0 & 57 & 1 & 59 & $20.0 \%$ & $0.34[0.01,8.29]$ & \\
\hline TRAPS 2016 & 8 & 59 & 0 & 61 & $23.5 \%$ & $17.57[1.04,297.67]$ & \\
\hline Total (95\% CI) & & 211 & & 215 & $100.0 \%$ & $2.34[0.43,12.74]$ & \\
\hline Total events: & 20 & & 7 & & & & \\
\hline \multicolumn{7}{|c|}{ Heterogeneity: $\mathrm{Tau}^{2}=1.09 ; \mathrm{Chi}^{2}=3.65, \mathrm{df}=2(\mathrm{P}=0.16) ; \mathrm{I}^{2}=45 \%$} & 0.01 \\
\hline \multicolumn{6}{|c|}{ Test for overall effect: $\mathrm{Z}=0.99(\mathrm{P}=0.32)$} & & Favours NOAC \\
\hline
\end{tabular}

Test for subgroup differences: Not applicable

\section{Analysis 7.2. Comparison 7: Sensitivity analysis NOAC vs VKA, Outcome 2: Worst case any thromboembolic event at the longest follow-up}

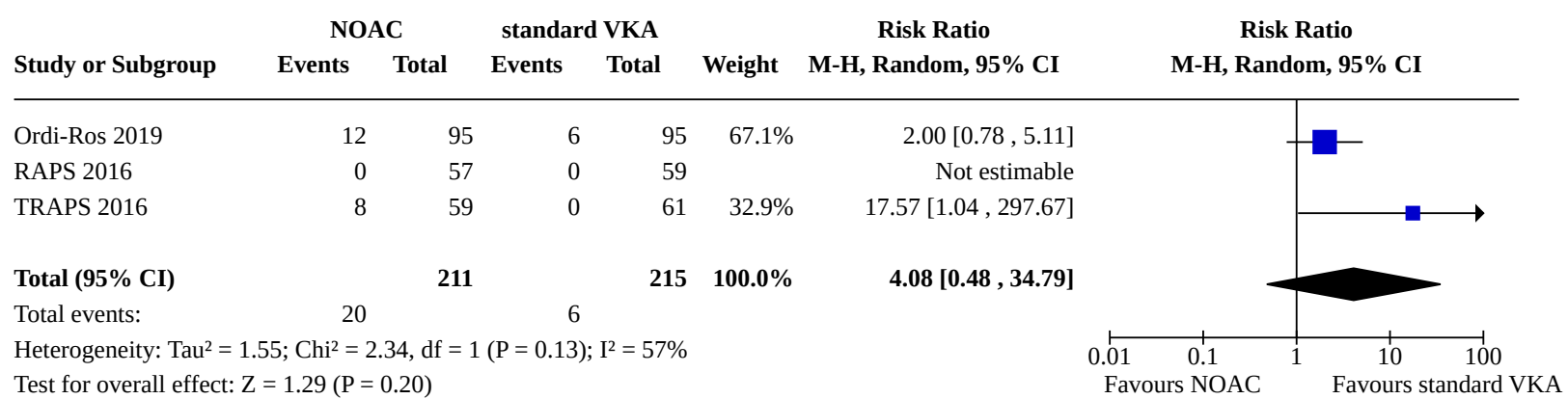

Test for subgroup differences: Not applicable

Analysis 7.3. Comparison 7: Sensitivity analysis NOAC vs VKA, Outcome 3: Best case major bleeding at the longest follow-up

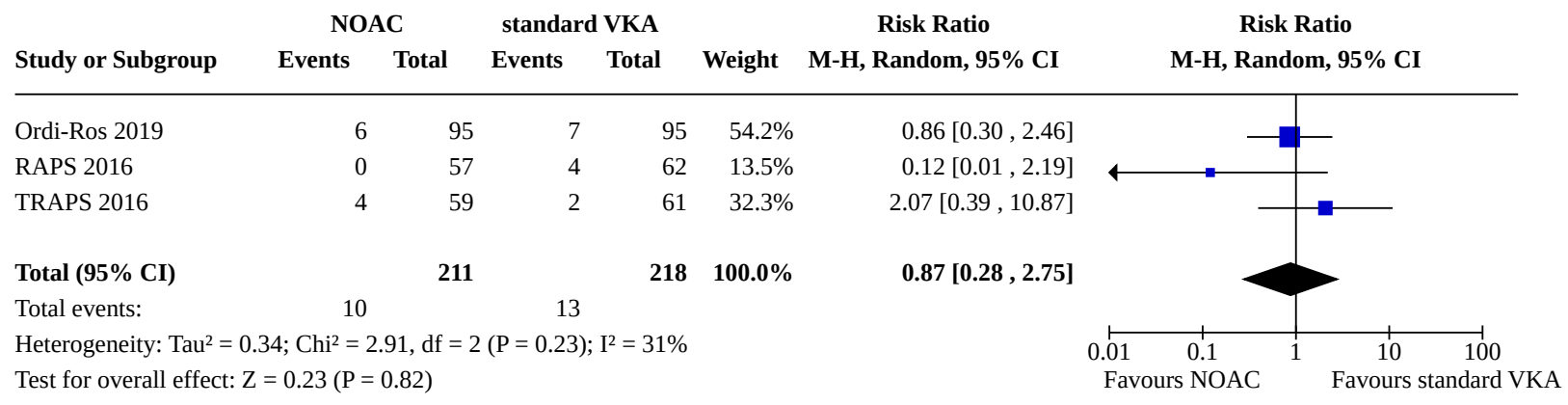


Analysis 7.4. Comparison 7: Sensitivity analysis NOAC vs VKA,

Outcome 4: Worst case major bleeding at the longest follow-up

\begin{tabular}{|c|c|c|c|c|c|c|c|}
\hline \multirow[b]{2}{*}{ Study or Subgroup } & \multicolumn{2}{|c|}{ NOAC } & \multicolumn{2}{|c|}{ standard VKA } & \multirow[b]{2}{*}{ Weight } & \multirow{2}{*}{$\begin{array}{c}\text { Risk Ratio } \\
\text { M-H Rndom } 95 \%\end{array}$} & \multirow{2}{*}{$\begin{array}{c}\text { Risk Ratio } \\
\text { M-H, Random, 95\% CI }\end{array}$} \\
\hline & Events & Total & Events & Total & & & \\
\hline Ordi-Ros 2019 & 6 & 95 & 7 & 95 & $71.3 \%$ & $0.86[0.30,2.46]$ & \\
\hline RAPS 2016 & 0 & 57 & 0 & 62 & & Not estimable & \\
\hline TRAPS 2016 & 4 & 59 & 2 & 61 & $28.7 \%$ & $2.07[0.39,10.87]$ & 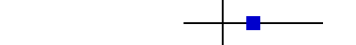 \\
\hline Total (95\% CI) & & 211 & & 218 & $100.0 \%$ & $1.10[0.45,2.68]$ & \\
\hline Total events: & 10 & & 9 & & & & \\
\hline Heterogeneity: $\mathrm{Tau}^{2}=$ & $00 ; \mathrm{Chi}^{2}=\mathrm{C}$ & $.77, \mathrm{df}=1$ & $1(\mathrm{P}=0.38)$ & $I^{2}=0 \%$ & & & $0.01 \quad 0.1$ \\
\hline
\end{tabular}

Test for subgroup differences: Not applicable

Analysis 7.5. Comparison 7: Sensitivity analysis NOAC vs VKA, Outcome 5: Best case all-cause death at the longest follow-up

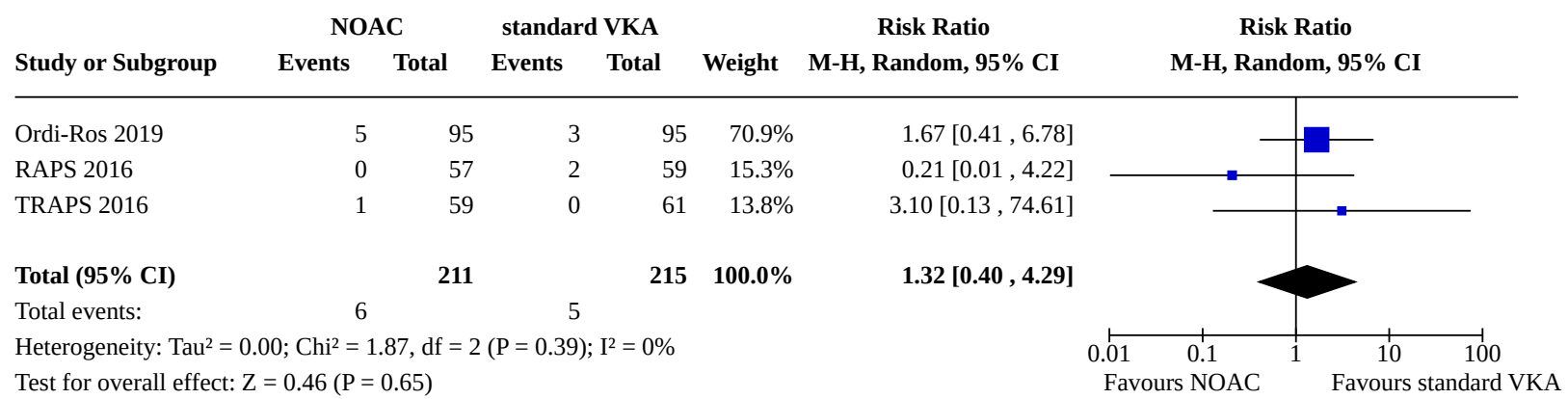

Test for subgroup differences: Not applicable

Analysis 7.6. Comparison 7: Sensitivity analysis NOAC vs VKA, Outcome 6: Worst case all-cause death at the longest follow-up

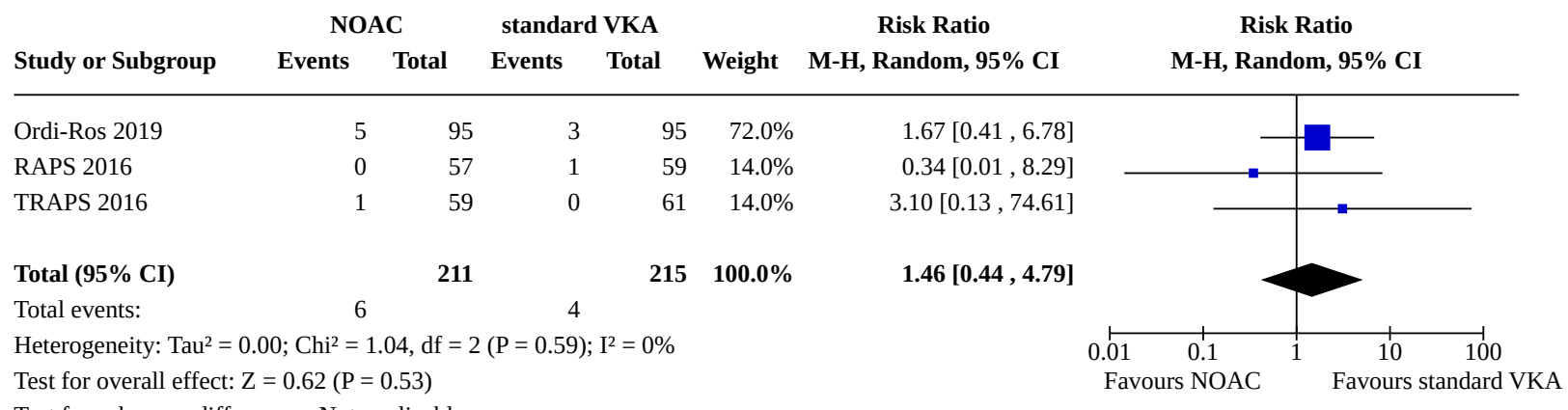


Analysis 7.7. Comparison 7: Sensitivity analysis NOAC vs VKA, Outcome 7:

Best case clinically relevant non-major bleeding at the longest follow up

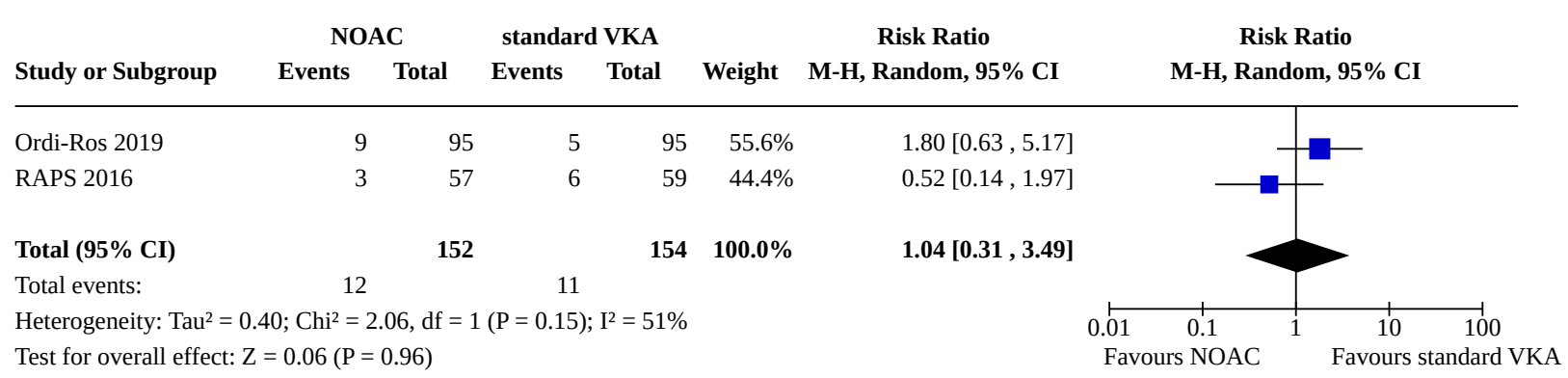

Test for subgroup differences: Not applicable

Analysis 7.8. Comparison 7: Sensitivity analysis NOAC vs VKA, Outcome 8: Worst case clinically relevant non-major bleeding at the longest follow up

\begin{tabular}{|c|c|c|c|c|c|c|c|c|}
\hline \multirow[b]{2}{*}{ Study or Subgroup } & \multicolumn{2}{|c|}{ NOAC } & \multicolumn{2}{|c|}{ standard VKA } & \multirow[b]{2}{*}{ Weight } & \multirow{2}{*}{$\begin{array}{c}\text { Risk Ratio } \\
\text { M-H, Random, 95\% CI }\end{array}$} & \multirow{2}{*}{$\begin{array}{c}\text { Risk Ratio } \\
\text { M-H, Random, 95\% CI }\end{array}$} & \\
\hline & Events & Total & Events & Total & & & & \\
\hline Ordi-Ros 2019 & 9 & 95 & 5 & 95 & $73.4 \%$ & $1.80[0.63,5.17]$ & & \\
\hline RAPS 2016 & 3 & 57 & 2 & 59 & $26.6 \%$ & $1.55[0.27,8.95]$ & & \\
\hline Total (95\% CI) & & 152 & & 154 & $100.0 \%$ & $1.73[0.70,4.27]$ & & \\
\hline Total events: & 12 & & 7 & & & & & \\
\hline \multirow{2}{*}{\multicolumn{5}{|c|}{$\begin{array}{l}\text { Heterogeneity: } \mathrm{Tau}^{2}=0.00 ; \mathrm{Chi}^{2}=0.02, \mathrm{df}=1(\mathrm{P}=0.89) ; \mathrm{I}^{2}=0 \% \\
\text { Test for overall effect } \mathrm{Z}=1.19(\mathrm{P}=0.23)\end{array}$}} & & 0.01 & 0.1 & 100 \\
\hline & & & & & & Fav & urs NOAC & Adard \\
\hline
\end{tabular}

Test for subgroup differences: Not applicable

\section{Analysis 7.9. Comparison 7: Sensitivity analysis NOAC vs VKA, Outcome 9: Best case minor bleeding at the longest follow-up}

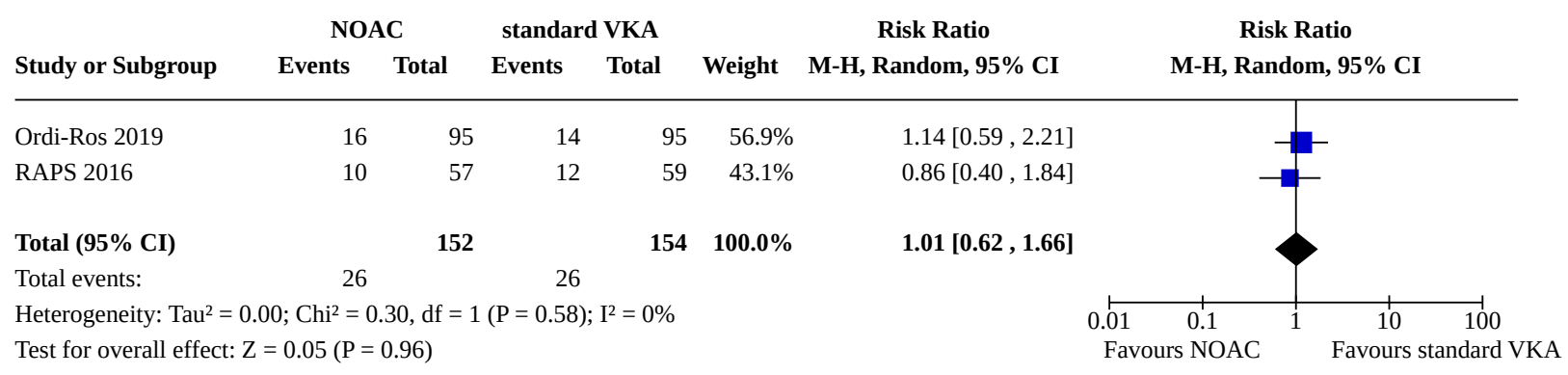


Analysis 7.10. Comparison 7: Sensitivity analysis NOAC vs VKA, Outcome 10: Worst case minor bleeding at the longest follow-up

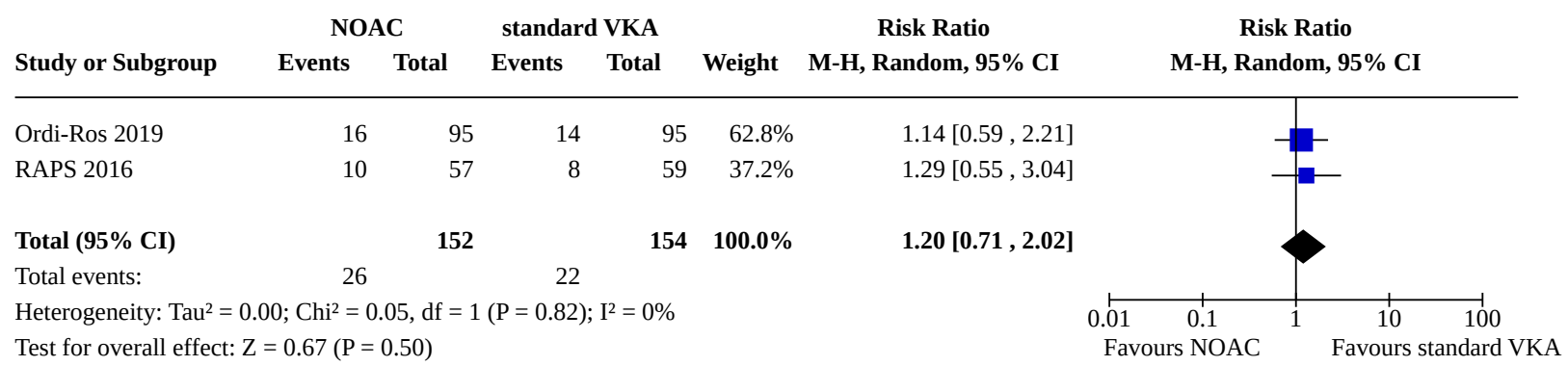

\section{APPEN DICES}

\section{Appendix 1. Appendix: CENTRAL search strategy}

ID Search Hits

\#1 MeSH descriptor: [Anticoagulants] this term only

\#2 MeSH descriptor: [Blood Coagulation Factors] explode all trees

\#3 MeSH descriptor: [Blood Coagulation] this term only

\#4 (anticoagul ${ }^{\star}$ or antithromb ${ }^{\star}$ ):ti,ab,kw (Word variations have been searched)

\#5 MeSH descriptor: [Warfarin] 3 tree(s) exploded

\#6 MeSH descriptor: [4-Hydroxycoumarins] explode all trees

\#7 MeSH descriptor: [Coumarins] this term only

\#8 MeSH descriptor: [Phenindione] 3 tree(s) exploded

\#9 (warfarin* or coumadin* or coumarin* or cumarin* or phenprocoum ${ }^{\star}$ or phenprocum ${ }^{\star}$ or dicoumar ${ }^{\star}$ or dicumar $^{\star}$ or acenocoumar $^{\star}$ or acenocumar ${ }^{\star}$ or fluindione or phenindione or clorindione or diphenadione or ethyl biscoumacetate):ti,ab,kw (Word variations have been searched)

\#10 (Vitamin K antagonist* or VKA or VKAs or antivitamin K):ti,ab,kw (Word variations have been searched)

\#11 MeSH descriptor: [Antithrombins] explode all trees

\#12 MeSH descriptor: [Thrombin] explode all trees

\#13 ((direct* near thrombin near inhib*) or DTI*1):ti,ab,kw (Word variations have been searched)

\#14 (argatroban or MD805 or MD-805 or dabigatran or ximelagatran or melagatran or efegatran or flovagatran or inogatran or napsagatran or bivalirudin or lepirudin or hirudin* or desirudin or desulfatohirudin or hirugen or hirulog or AZD0837 or bothrojaracin or odiparcil):ti,ab,kw (Word variations have been searched)

\#15 MeSH descriptor: [Factor Xa] explode all trees

\#16 ((factor Xa or factor 10a or fXa or autoprothrombin c or thrombokinase) near/5 inhib^):ti,ab,kw (Word variations have been searched)

\#17 (activated near/5 (factor X or factor 10) near/5 inhib ${ }^{\star}$ ):ti,ab,kw (Word variations have been searched)

\#18 xabans:ti,ab,kw (Word variations have been searched)

\#19 (antistasin or apixaban or betrixaban or du 176b or eribaxaban or fondaparinux or idraparinux or otamixaban or razaxaban or rivaroxaban or yagin or ym 150 or ym150 or LY517717 or darexaban or edoxaban or SSR126517E or fidexaban or idrabiotaparinux or letaxaban or tanogitran or taxexaban):ti,ab,kw (Word variations have been searched)

Antiplatelet and anticoagulant agents for secondary prevention of stroke and other thromboembolic events in people with 
\#20 MeSH descriptor: [Heparin] explode all trees

\#21 (heparin* or Imwh* or enoxaparin* or glycosaminoglycan* or nadroparin* or mesoglycan* or tedelparin* or certoparin or tinzaparin or parnaparin or dalteparin or reviparin or fraxiparin* or danaparoid or lomoparan or org 10172 or mesoglycan or pentosan polysul ${ }^{\star}$ or sp54

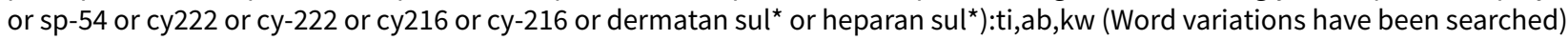

$\# 22\{$ or \#1-\#21\}

\#23 MeSH descriptor: [Platelet Aggregation Inhibitors] this term only

\#24 MeSH descriptor: [Cyclooxygenase Inhibitors] explode all trees

\#25 MeSH descriptor: [Thienopyridines] explode all trees

\#26 MeSH descriptor: [Phosphodiesterase Inhibitors] explode all trees

\#27 MeSH descriptor: [Thromboxane A2] explode all trees

\#28 MeSH descriptor: [Purinergic P2Y Receptor Antagonists] this term only

\#29 MeSH descriptor: [Platelet Activation] explode all trees

\#30 MeSH descriptor: [Blood Platelets] explode all trees and with qualifier(s): [Drug effects - DE]

\#31 (antiplatelet* or anti-platelet* or antithrombocytic or anti-thrombocytic):ti,ab,kw (Word variations have been searched)

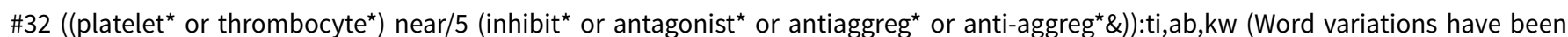
searched)

\#33 (cyclooxygenase inhibitor ${ }^{\star}$ or thienopyridine ${ }^{\star}$ or phosphodiesterase inhibitor ${ }^{\star}$ ):ti,ab,kw (Word variations have been searched)

\#34 (thromboxane A2 near/3 (inhib* or antag*)):ti,ab,kw (Word variations have been searched)

\#35 (aspirin* or acetyl salicylic acid* or acetyl?salicylic acid*):ti,ab,kw (Word variations have been searched)

\#36 (ARC1779 or AZD6140 or alprostadil or asasantin or carnitine or cilostazol or clopidogrel or cloricromene or cv4151 or cV-4151 or defibrotide or dilazep or dipyridamol* or disintegrin* or ditazol or E5880 or E5510 or epoprostenol* or fluribrofen or fut-175 or iloprost* or indobufen or isbogrel or kbt3022 or kbt-3022 or ketanserin ${ }^{\star}$ or ketoprofen or ketorolac or levamisol* or ligustrazine* or tromethamine* or milrinone* or mopidamol* or naudicelle or nimesulide or ozagrel ${ }^{\star}$ or oky046 or oky-046 or oky-1581 or phthalzinol or picotamide or policosanol or prasugrel or procainamide or sarpogrelate or satigrel or sulphinpyrazone or sulfinpyrazone or suloctadil or terutroban or ticagrelor or ticlopidine or trapidil or triflusal or vorapaxar):ti,ab,kw (Word variations have been searched)

\#37 \{or \#23-\#36\}

\#38\#22 or 37

\#39 MeSH descriptor: [Antiphospholipid Syndrome] this term only

\#40 MeSH descriptor: [Phospholipids] explode all trees

\#41 MeSH descriptor: [Phospholipids] explode all trees

\#42 MeSH descriptor: [Cardiolipins] this term only

\#43 MeSH descriptor: [Antibodies, Antiphospholipid] explode all trees

\#44 ((antiphospholipid or anti-phospholipid or phospholipid or anti-cardiolipin or anticardiolipin or cardiolipin or beta 2-glycoprotein I) near $/ 5$ (auto* or antibod* or syndrome or inhibit\$)):ti,ab,kw (Word variations have been searched)

\#45 (APS or APLS or aCLIN):ti,ab,kw (Word variations have been searched)

\#46 (lupus near/5 (coagulant* or inhibit* or antibod $\left.{ }^{\star}\right)$ ):ti,ab,kw (Word variations have been searched)

\#47 Ashersons syndrome:ti,ab,kw (Word variations have been searched)

\#48 Hughes syndrome:ti,ab,kw (Word variations have been searched)

\#49 MeSH descriptor: [beta 2-Glycoprotein I] explode all trees

Antiplatelet and anticoagulant agents for secondary prevention of stroke and other thromboembolic events in people with antiphospholipid syndrome (Review)

Copyright (C) 2020 The Cochrane Collaboration. Published by John Wiley \& Sons, Ltd. 
\#50 MeSH descriptor: [Glycoproteins] this term only

\#51 "beta 2-glycoprotein I":ti,ab,kw (Word variations have been searched)

\#52 \{or \#39-\#51\}

$\# 53 \# 38$ and \#52

\section{Appendix 2. Appendix: MEDLINE search strategy}

1. exp anticoagulants/

2. exp Blood coagulation factors/ai, de or exp Blood coagulation/ai, de

3. (anticoagul\$ or antithromb\$).tw.

4. Warfarin/ or 4-hydroxycoumarins/ or acenocoumarol/ or coumarins/ or dicumarol/ or ethyl biscoumacetate/ or phenindione/ or phenprocoumon/

5. exp Vitamin K/ai

6. (warfarin $\$$ or coumadin $\$$ or coumarin $\$$ or cumarin $\$$ or phenprocoum $\$$ or phenprocum $\$$ or dicoumar $\$$ or dicumar\$ or acenocoumar\$ or acenocumar $\$$ or fluindione or phenindione or clorindione or diphenadione or ethyl biscoumacetate).tw, nm.

7. (Vitamin $\mathrm{K}$ antagonist\$ or VKA or VKAs or antivitamin K).tw.

8. exp antithrombins/ or hirudin therapy/ or thrombin/ai

9. ((direct\$ adj5 thrombin adj5 inhib\$) or DTI\$1).tw.

10. (argatroban or MD805 or MD-805 or dabigatran or ximelagatran or melagatran or efegatran or flovagatran or inogatran or napsagatran or bivalirudin or lepirudin or hirudin\$ or desirudin or desulfatohirudin or hirugen or hirulog or AZD0837 or bothrojaracin or odiparcil).tw,nm.

11. factor $\mathrm{Xa} /$

12. ((factor Xa or factor 10a or fXa or autoprothrombin c or thrombokinase) adj5 inhib\$).tw.

13. (activated adj5 (factor $X$ or factor 10$)$ adj5 inhib\$).tw.

14. xabans.tw.

15. (antistasin or apixaban or betrixaban or du $176 \mathrm{~b}$ or eribaxaban or fondaparinux or idraparinux or otamixaban or razaxaban or rivaroxaban or yagin or ym 150 or ym150 or LY517717 or darexaban or edoxaban or SSR126517E or fidexaban or idrabiotaparinux or letaxaban or tanogitran or taxexaban).tw,nm.

16. heparin/ or exp heparin, low-molecular-weight/ or heparinoids/

17. (heparin or Imwh\$ or enoxaparin or glycosaminoglycan\$ or nadroparin\$ or mesoglycan\$ or tedelparin\$ or certoparin or tinzaparin or parnaparin or dalteparin or reviparin or fraxiparin $\$$ or danaparoid or lomoparan or org 10172 or mesoglycan or pentosan polysul\$ or sp54 or sp-54 or cy222 or cy-222 or cy216 or cy-216 or dermatan sul\$ or heparan sul\$).tw,nm.

18. 1 or 2 or 3 or 4 or 5 or 6 or 7 or 8 or 9 or 10 or 11 or 12 or 13 or 14 or 15 or 16 or 17

19. exp Platelet aggregation inhibitors/

20. exp Cyclooxygenase Inhibitors/ or exp Thienopyridines/ or exp Phosphodiesterase Inhibitors/ or Thromboxane A2/ai or exp Purinergic P2Y Receptor Antagonists/

21. exp Platelet activation/de

22. exp Blood Platelets/de

23. (antiplatelet\$ or anti-platelet\$ or antithrombocytic or anti-thrombocytic).tw.

24. ((platelet\$ or thrombocyte\$) adj5 (inhibit\$ or antagonist\$ or antiaggreg\$ or anti-aggreg\$)).tw.

25. (cyclooxygenase inhibitor\$ or thienopyridine\$ or phosphodiesterase inhibitor\$).tw.

Antiplatelet and anticoagulant agents for secondary prevention of stroke and other thromboembolic events in people with

Copyright (c) 2020 The Cochrane Collaboration. Published by John Wiley \& Sons, Ltd. 
26. (thromboxane A2 adj3 (inhib\$ or antag\$)).tw.

27. (aspirin\$ or acetyl salicylic acid\$ or acetyl?salicylic acid\$).tw, nm.

28. (ARC1779 or AZD6140 or alprostadil or asasantin or carnitine or cilostazol or clopidogrel or cloricromene or cV4151 or CV-4151 or defibrotide or dilazep or dipyridamol\$ or disintegrin\$ or ditazol or E5880 or E5510 or epoprostenol\$ or fluribrofen or fut-175 or iloprost\$ or indobufen or isbogrel or kbt3022 or kbt-3022 or ketanserin\$ or ketoprofen or ketorolac or levamisol\$ or ligustrazine\$ or tromethamine $\$$ or milrinone $\$$ or mopidamol\$ or naudicelle or nimesulide or ozagrel\$ or oky046 or oky-046 or oky-1581 or phthalzinol or picotamide or policosanol or prasugrel or procainamide or sarpogrelate or satigrel or sulphinpyrazone or sulfinpyrazone or suloctadil or terutroban or ticagrelor or ticlopidine or trapidil or triflusal or vorapaxar).tw,nm.

\section{19 or 20 or 21 or 22 or 23 or 24 or 25 or 26 or 27 or 28}

30. Antiphospholipid Syndrome/

31. Phospholipids/

32. Cardiolipins/

33. antibodies, antiphospholipid/ or antibodies, anticardiolipin/ or lupus coagulation inhibitor/

34. ((antiphospholipid or anti-phospholipid or phospholipid or anti-cardiolipin or anticardiolipin or cardiolipin or beta 2-glycoprotein I) adj5 (auto\$ or antibod\$ or syndrome or inhibit\$)).tw.

35. (APS or APLS or aCLIN).tw.

36. (lupus adj5 (coagulant\$ or inhibit\$ or antibod\$)).tw.

37. Ashersons syndrome.tw.

38. Hughes syndrome.tw.

39. beta 2-Glycoprotein I/ or Glycoproteins/

40. beta 2-Glycoprotein I.tw.

41.30 or 33 or 34 or 35 or 36 or 37 or 38 or 39 or 40

42. 18 or 29

43. 41 and 42

44. Randomized Controlled Trials as Topic/

45. Random Allocation/

46. Controlled Clinical Trials as Topic/

47. control groups/

48. clinical trials as topic/ or clinical trials, phase i as topic/ or clinical trials, phase ii as topic/or clinical trials, phase iii as topic/ or clinical trials, phase iv as topic/

49. double-blind method/

50. single-blind method/

51. Placebos/

52. placebo effect/

53. Drug Evaluation/

54. Research Design/

55. randomized controlled trial.pt.

56. controlled clinical trial.pt.

Antiplatelet and anticoagulant agents for secondary prevention of stroke and other thromboembolic events in people with antiphospholipid syndrome (Review)

Copyright (c) 2020 The Cochrane Collaboration. Published by John Wiley \& Sons, Ltd. 
57. (clinical trial or clinical trial phase i or clinical trial phase ii or clinical trial phase iii or clinical trial phase iv).pt.

58. (random\$ or RCT or RCTs).tw.

59. (controlled adj5 (trial\$ or stud\$)).tw.

60. (clinical\$ adj5 trial\$).tw.

61. ((control or treatment or experiment $\$$ or intervention) adj5 (group\$ or subject\$ or patient\$)).tw.

62. (quasi-random\$ or quasi random\$ or pseudo-random\$ or pseud or random\$).tw.

63. ((singl\$ or doubl\$ or tripl\$ or trebl\$) adj5 (blind\$ or mask\$)).tw.

64. placebo\$.tw.

65. controls.tw.

66. exp animals/ not humans.sh.

67.44 or 45 or 46 or 47 or 48 or 49 or 50 or 51 or 52 or 53 or 54 or 55 or 56 or 57 or 58 or 59 or 60 or 61 or 62 or 63 or 64 or 65

68.67 not 66

69.43 and 67

70.43 and 68

\section{Appendix 3. Appendix: Embase search strategy}

1. exp anticoagulant agent/ or exp anticoagulant therapy/

2. exp blood clotting/

3. exp blood clotting factor/

4. (anticoagul\$ or antithromb\$).tw.

5. exp coumarin derivative/

6. (warfarin $\$$ or coumadin $\$$ or coumarin $\$$ or cumarin $\$$ or phenprocoum $\$$ or phenprocum $\$$ or dicoumar $\$$ or dicumar $\$$ or acenocoumar $\$$ or acenocumar\$ or fluindione or phenindione or clorindione or diphenadione or ethyl biscoumacetate).tw.

7. exp vitamin $\mathrm{K}$ group/

8. (Vitamin $\mathrm{K}$ antagonist\$ or VKA or VKAs or antivitamin K).tw.

9. ((direct\$ adj5 thrombin adj5 inhib\$) or DTI\$1).tw.

10. (argatroban or MD805 or MD-805 or dabigatran or ximelagatran or melagatran or efegatran or flovagatran or inogatran or napsagatran or bivalirudin or lepirudin or hirudin\$ or desirudin or desulfatohirudin or hirugen or hirulog or AZD0837 or bothrojaracin or odiparcil).tw.

11. ((factor Xa or factor 10a or fXa or autoprothrombin c or thrombokinase) adj5 inhib\$).tw.

12. (antistasin or apixaban or betrixaban or du $176 \mathrm{~b}$ or eribaxaban or fondaparinux or idraparinux or otamixaban or razaxaban or rivaroxaban or yagin or ym 150 or ym150 or LY517717 or darexaban or edoxaban or SSR126517E or fidexaban or idrabiotaparinux or letaxaban or tanogitran or taxexaban).tw.

13. xabans.tw.

14. (heparin\$ or Imwh\$ or enoxaparin\$ or glycosaminoglycan\$ or nadroparin $\$$ or mesoglycan\$ or tedelparin\$ or certoparin or tinzaparin or parnaparin or dalteparin or reviparin or fraxiparin\$ or danaparoid or lomoparan or org 10172 or mesoglycan or pentosan polysul\$ or sp54 or sp-54 or cy222 or cy-222 or cy216 or cy-216 or dermatan sul\$ or heparan sul\$).tw.

\section{5. or/1-14}

16. exp antithrombocytic agent/

Antiplatelet and anticoagulant agents for secondary prevention of stroke and other thromboembolic events in people with antiphospholipid syndrome (Review)

Copyright (C) 2020 The Cochrane Collaboration. Published by John Wiley \& Sons, Ltd. 


\section{7. exp thrombocyte aggregation/}

18. exp thrombocyte aggregation inhibition/

19. thienopyridine derivative/

20. exp prostaglandin synthase inhibitor/ or thienopyridine derivative/ or exp phosphodiesterase inhibitor/ or thromboxane A2/ or purinergic P2Y receptor antagonist/

21. thrombocyte activation/

22. exp thrombocyte/

23. (antiplatelet\$ or anti-platelet\$ or antithrombocytic or anti-thrombocytic).tw.

24. ((platelet\$ or thrombocyte\$) adj5 (inhibit\$ or antagonist\$ or antiaggreg\$ or anti-aggreg\$)).tw.

25. (cyclooxygenase inhibitor\$ or thienopyridine\$ or phosphodiesterase inhibitor\$).tw.

26. (thromboxane A2 adj3 (inhib\$ or antag\$)).tw.

27. (aspirin\$ or acetyl salicylic acid\$ or acetyl?salicylic acid\$).tw.

28. (ARC1779 or AZD6140 or alprostadil or asasantin or carnitine or cilostazol or clopidogrel or cloricromene or cV4151 or cV-4151 or defibrotide or dilazep or dipyridamol\$ or disintegrin\$ or ditazol or E5880 or E5510 or epoprostenol\$ or fluribrofen or fut-175 or iloprost\$ or indobufen or isbogrel or kbt3022 or kbt-3022 or ketanserin\$ or ketoprofen or ketorolac or levamisol\$ or ligustrazine\$ or tromethamine $\$$ or milrinone or mopidamol\$ or naudicelle or nimesulide or ozagrel\$ or oky046 or oky-046 or oky-1581 or phthalzinol or picotamide or policosanol or prasugrel or procainamide or sarpogrelate or satigrel or sulphinpyrazone or sulfinpyrazone or suloctadil or terutroban or ticagrelor or ticlopidine or trapidil or triflusal or vorapaxar).tw.

29. 0 r/16-28

30. antiphospholipid syndrome/

31. phospholipid/ or exp phospholipid antibody/

32. cardiolipin/ or exp cardiolipin antibody/

33. lupus anticoagulant/

34. ((antiphospholipid or anti-phospholipid or phospholipid or anti-cardiolipin or anticardiolipin or cardiolipin or beta 2-glycoprotein I) $\operatorname{adj} 5$ (auto\$ or antibod\$ or syndrome or inhibit\$)).tw.

35. (APS or APLS or aCLIN).tw.

36. (lupus adj5 (coagulant\$ or inhibit\$ or antibod\$)).tw.

37. (Ashersons syndrome or hughes syndrome).tw.

38. glycoprotein/ or beta2 glycoprotein 1/ or exp beta2 glycoprotein 1 antibody/

39. or $/ 30-38$

40. Randomized Controlled Trial/ or "randomized controlled trial (topic)"/

41. Randomization/

42. Controlled clinical trial/ or "controlled clinical trial (topic)"/

43. control group/ or controlled study/

44. clinical trial/ or "clinical trial (topic)"/ or phase 1 clinical trial/ or phase 2 clinical trial/ or phase $3 \mathrm{clinical}$ trial/ or phase $4 \mathrm{clinical}$ trial/

45. Crossover Procedure/

46. Double Blind Procedure/

47. Single Blind Procedure/ or triple blind procedure/

Antiplatelet and anticoagulant agents for secondary prevention of stroke and other thromboembolic events in people with antiphospholipid syndrome (Review)

Copyright (C) 2020 The Cochrane Collaboration. Published by John Wiley \& Sons, Ltd. 
48. placebo/ or placebo effect/

49. (random\$ or RCT or RCTs).tw.

50. (controlled adj5 (trial\$ or stud\$)).tw.

51. (clinical\$ adj5 trial\$).tw.

52. ((control or treatment or experiment\$ or intervention) adj5 (group\$ or subject\$ or patient\$)).tw.

53. (quasi-random\$ or quasi random $\$$ or pseudo-random $\$$ or pseudo random\$).tw.

54. ((control or experiment\$ or conservative) adj5 (treatment or therapy or procedure or manage\$)).tw.

55. ((singl\$ or doubl\$ or tripl\$ or trebl\$) adj5 (blind\$ or mask\$)).tw.

56. (cross-over or cross over or crossover).tw.

57. (placebo\$ or sham).tw.

58. trial.ti.

59. (assign\$ or allocat\$).tw.

60. controls.tw.

61. or $/ 40-60$

62. 15 or 29

63. 39 and 61 and 62

64. (exp animals/ or exp invertebrate/ or animal experiment/ or animal model/or animal tissue/ or animal cell/ or nonhuman/) not (human/ or normal human/ or human cell/)

65.63 not 64

\section{Appendix 4. Appendix: Trials registries search strategies}

\section{ClinicalTrials.gov (www.clinicaltrials.gov)}

Antiphospholipid AND Syndrome AND EXACT "Interventional" [STUDY-TYPES]

Hughes AND Syndrome AND EXACT "Interventional" [STUDY-TYPES]

Asherson AND Syndrome AND EXACT "Interventional" [STUDY-TYPES]

Antiphospholipid Syndrome AND anticoagulant

Antiphospholipid syndrome AND antiplatelet

Antiphospholipid Syndrome AND warfarin

Antiphospholipid Syndrome AND apixaban

Antiphospholipid Syndrome AND dabigatran

Antiphospholipid Syndrome AND rivaroxaban

Antiphospholipid Syndrome AND heparin

Antiphospholipid Syndrome AND RCT

Antiphospholipid AND intervention

Antiphospholipid AND anticoagulant

Antiphospholipid AND antiplatelet 
Antiphospholipid AND warfarin

Antiphospholipid AND apixaban

Antiphospholipid AND dabigatran

Antiphospholipid AND rivaroxaban

Antiphospholipid AND heparin

Antiphospholipid AND RCT

Stroke Trials Registry (www.strokecenter.org/trials).

Antiphospholipid syndrome

antiphosphoipid antibody

Hughes syndrome

Asherson syndrome

European Trials Register (www.clinicaltrialsregister.eu).

Antiphospholipid AND Syndrome

Hughes AND Syndrome

Asherson AND Syndrome

Antiphospholipid AND Antibody

Antiphospholipid Syndrome AND anticoagulant

Antiphospholipid syndrome AND intervention

Antiphospholipid syndrome AND antiplatelet

Antiphospholipid Syndrome AND warfarin

Antiphospholipid Syndrome AND apixaban

Antiphospholipid Syndrome AND dabigatran

Antiphospholipid Syndrome AND rivaroxaban

Antiphospholipid Syndrome AND heparin

Antiphospholipid Syndrome AND RCT

4) ISRCTN Registry (http://www.isrctn.com/).

filter 'condition': Antiphospholipid syndrome

filter 'condition': Hughes syndrome

filter 'condition': Asherson syndrome

The World Health Organization (WHO) International Trials Registry Platform

Antiphospholipid AND Syndrome

Hughes AND Syndrome

Asherson AND Syndrome

Antiphospholipid Syndrome AND anticoagulant

Antiphospholipid syndrome AND intervention

Antiplatelet and anticoagulant agents for secondary prevention of stroke and other thromboembolic events in people with

Copyright (c) 2020 The Cochrane Collaboration. Published by John Wiley \& Sons, Ltd. 
Antiphospholipid syndrome AND antiplatelet

Antiphospholipid Syndrome AND warfarin

Antiphospholipid Syndrome AND apixaban

Antiphospholipid Syndrome AND dabigatran

Antiphospholipid Syndrome AND rivaroxaban

Antiphospholipid Syndrome AND heparin

Antiphospholipid Syndrome AND RCT

Antiphospholipid AND intervention

Antiphospholipid AND anticoagulant

Antiphospholipid AND antiplatelet

Antiphospholipid AND warfarin

Antiphospholipid AND apixaban

Antiphospholipid AND dabigatran

Antiphospholipid AND rivaroxaban

Antiphospholipid AND heparin

Antiphospholipid AND RCT

WHAT'S NEW

\begin{tabular}{lll}
\hline Date & Event & Description \\
\hline 19 February 2020 & New search has been performed & $\begin{array}{l}\text { Updated search; we included three new studies giving a total of } \\
\text { eight studies with } 811 \text { randomized participants. }\end{array}$ \\
\hline 19 February 2020 & $\begin{array}{l}\text { New citation required and conclusions } \\
\text { have changed }\end{array}$ & $\begin{array}{l}\text { We identified more evidence on NOAC (rivaroxaban). Therefore, } \\
\text { our conclusions have become more specific. We identified evi- } \\
\text { dence on the effect of VKA combined with AP agent compared } \\
\text { with VKA, which was not included in the previous version of the } \\
\text { review. Conclusions did not change for other comparisons. }\end{array}$ \\
\hline
\end{tabular}

\section{H ISTORY}

Protocol first published: Issue 4, 2016

Review first published: Issue 10, 2017

\section{CONTRIBUTIONS OF AUTHORS}

MMB, MCL, and AU developed the concept of the study, and MMB, AP, and WS developed the search strategy. MMB, MCL, WS, AP, MK, and MJS participated in title and abstract screening, full-text screening, and data extraction. MMB and MC-L participated in data analyses; MMB, MC-L, WS, and AU participated in data interpretation. All review authors contributed to the preparation of the review text and have agreed upon this final version.

\section{DECLARATIONS OF INTEREST}

Malgorzata M Bala: received honoraria as a freelancer from a company doing health technology assessments and systematic reviews for various clients; she has not been involved in any work funded by companies who are manufacturers or marketers of the interventions of interest or potential comparators; she is not aware of any direct conflict of interest. 
Magdalena Celinska-Lowenhoff: participates in clinical trials not related to the topic of this review. Wojciech Szot: participates in clinical trials not related to the topic of this review.

Agnieszka Padjas: none known.

Mateusz Kaczmarczyk: none known.

Mateusz Swierz: none known.

Anetta Undas: lecture honoraria from Boehringer Ingelheim, Bayer Pharma AG, and Pfizer/Bristol-Meyers-Squibb within the previous three years. Personal fees from the publisher Medycyna Praktyczna.

\section{SOURCES OF SUPPORT}

\section{Internal sources}

- Jagiellonian University Medical College, Poland

All the review authors are either employed by JUMC or are students at JUMC and worked on the review within their research activities

\section{External sources}

- Ministry of Science and Higher Education, Poland

K/ZDS/006101; Core funding for statutory R \& D activities

\section{DIFFERENCES BETWEEN PROTOCOLANDREVIEW}

Changes in people responsible for the conduct of the review: additional help from two other authors and trainee reviewers in title and abstract screening, full-text screening, data extraction and risk of bias assessment, consulting as third review authors.

Use of Microsoft Excel spreadsheet instead of Covidence for data extraction due to insufficient flexibility of Covidence data extraction tables.

In the protocol we stated: "If possible, we planned to explore heterogeneity by subgroup analyses taking into account the type of drug (anticoagulant, antiplatelet, combination); dose of the active treatment; APS diagnostic criteria; single-, double- and triple-antibody positivity; lupus anticoagulant positivity versus other antibodies; time-based versus tissue-based definition of TIA; presence versus absence of traditional cardiovascular risk factors; type of index event (arterial versus venous); and INR value at thrombotic event."

In this update we changed this to:

"If possible, we planned to explore heterogeneity by subgroup analyses taking into account single-, double- and triple-antibody positivity; lupus anticoagulant positivity versus other antibodies; presence versus absence of traditional cardiovascular risk factors; type of index event (arterial versus venous)."

We planned subgroup analyses for all outcomes only if there was sufficient number of studies, i.e. for a single analysis at least six included studies with the information relevant for subgroup analysis (Deeks 2001).

In the review we presented results by comparisons separately for anticoagulants, antiplatelets, and different sets of their combinations.

\section{N DEX TERMS}

\section{Medical Subject Headings (MeSH)}

Anticoagulants [adverse effects] [ ${ }^{\star}$ therapeutic use]; Antiphospholipid Syndrome [ ${ }^{\star}$ complications]; Cause of Death; Factor Xa Inhibitors [therapeutic use]; Hemorrhage [chemically induced]; Platelet Aggregation Inhibitors [adverse effects] [ ${ }^{*}$ therapeutic use]; Randomized Controlled Trials as Topic; Rivaroxaban [therapeutic use]; ${ }^{*}$ Secondary Prevention; Stroke [mortality] [ ${ }^{*}$ prevention \& control]; Thromboembolism [mortality] [prevention \& control]; Warfarin [therapeutic use]

\section{MeSH check words}

Humans 